\title{
A THOUGHT ON GENERALIZED MORREY SPACES
}

\author{
YOSHIHIRO SAWANO \\ Department of Mathematics and Information Science, \\ Tokyo Metropolitan University, Minamioosawa, \\ Hachouji-city 1-1, Tokyo 192-0364, Japan. \\ ysawano@tmu.ac.jp
}

\begin{abstract}
Morrey spaces can complement the boundedness properties of operators that Lebesgue spaces can not handle. Morrey spaces which we have been handling are called classical Morrey spaces. However, classical Morrey spaces are not totally enough to describe the boundedness properties. To this end, we need to generalize parameters $p$ and $q$, among others $p$.

Key words and phrases: Morrey spaces, generalized Morrey spaces, boundedness properties of operators.
\end{abstract}

\begin{abstract}
Abstrak. Ruang Morrey dapat menggenapi sifat keterbatasan operator yang tidak dipenuhi di ruang Lebesgue. Ruang Morrey yang telah dikaji adalah ruang Morrey klasik. Namun, ruang Morrey klasik tidak cukup lengkap untuk mengungkapkan sifat keterbatasan. Untuk itu, kita perlu memperumum parameter $p$ dan $q$, terutama

Kata kunci: Ruang Morrey, ruang Morrey diperumum, sifat keterbatasan operator.
\end{abstract}

\section{INTRODUCTION}

This note studies the function spaces which Guliyev, Mizuhara and Nakai defined in $[28,69,73]$. Let $0<q \leq p<\infty$. We define the classical Morrey space $\mathcal{M}_{q}^{p}\left(\mathbb{R}^{n}\right)$ to be the set of all measurable functions $f$ for which the quantity

$$
\|f\|_{\mathcal{M}_{q}^{p}} \equiv \sup _{x \in \mathbb{R}^{n}, r>0}|Q(x, r)|^{\frac{1}{p}-\frac{1}{q}}\left(\int_{Q(x, r)}|f(y)|^{q} d y\right)^{\frac{1}{q}}
$$

2000 Mathematics Subject Classification: 26D15, 46B25, 46E30

Received: 27 October 2018, revised: 20 December 2018, accepted 29 December 2018. 
is finite, where $Q(x, r)=\left\{y \in \mathbb{R}^{n}:\left|x_{1}-y_{1}\right|,\left|x_{2}-y_{2}\right|, \ldots,\left|x_{n}-y_{n}\right| \leq r\right\}$. To describe the endpoint case or to describe the intersection space, sometimes it is useful to generalize the parameter $p$ : let us suppose that $p$ comes from the function $t^{\frac{n}{p}}$. So, we envisage the situation where $t^{\frac{n}{p}}$ is replaced by a general function $\varphi$. More precisely, it is known as the Adams theorem that $I_{\alpha}$ maps $\mathcal{M}_{q}^{p}\left(\mathbb{R}^{n}\right)$ to $\mathcal{M}_{t}^{s}\left(\mathbb{R}^{n}\right)$ whenever $1<q \leq p<\infty$ and $1<t \leq s<\infty$ satisfy

$$
\frac{1}{s}=\frac{1}{p}-\frac{\alpha}{n}, \quad \frac{q}{p}=\frac{t}{s}
$$

Here $I_{\alpha}$ is the fractional integral operator given by (12) for a nonnegative measurable function $f: \mathbb{R}^{n} \rightarrow[0, \infty]$. However, it is known that we can not take $s=\infty$. In fact, $I_{\alpha}$ fails to be bounded from $\mathcal{M}_{q}^{n / \alpha}\left(\mathbb{R}^{n}\right)$ to $L^{\infty}\left(\mathbb{R}^{n}\right)$ for all $1 \leq q \leq \frac{n}{\alpha}$. To compensate for this failure, we can use generalized Morrey spaces. We will define the generalized Morrey norm by

$$
\|f\|_{\mathcal{M}_{q}^{\varphi}} \equiv \sup _{x \in \mathbb{R}^{n}, r>0} \frac{1}{\varphi(r)}\left(\frac{1}{|Q(x, r)|} \int_{Q(x, r)}|f(y)|^{q} d y\right)^{\frac{1}{q}}<\infty .
$$

Here $\varphi:(0, \infty) \rightarrow[0, \infty)$ is a function which does not vanish at least at some point. Another advantage of generalized Morrey spaces is that we can cover many function spaces related to Lebesgue spaces.

Although we do not consider the direct applications of generalized Morrey spaces to PDEs, generalized Morrey spaces can be applied to PDEs. Applications to the divergence elliptic differential equations can be found in [59]. Applications to the nondivergence elliptic differential equations can be found in $[35,114,123]$. Applications to the parabolic differential equations can be found in $[112,128]$. See $[30,42,113]$ for the parabolic oblique derivative problem. See [61] for applicatitons to Schrödinger equations. We refer to [64] for the application to singular integral equations.

This note is organized as follows: We will collect some preliminary facts in Section 2 In Section 3, we define generalized Morrey spaces and then discuss the structure of generalized Morrey spaces. The conditions on $\varphi$ will be important. So we discuss them quite carefully. Section 4 is a detailed discusssion of the boundedness properties of the operators. Here we handle the Hardy-Littlewood maximal operators, the Riesz potentails, the Riesz transforms and the fractional maximal operators as well as their generalizations. Section 5 is a survey of other related function spaces.

Here we list a series of (somewhat standard) notation we use in this note.

- Let $(X, d)$ be a metric space. We denote by $B(x, r)$ the ball centered at $x$ of radius $r$. Namely, we write

$$
B(x, r) \equiv\left\{y \in \mathbb{R}^{n}: d(x, y)<r\right\}
$$

when $x \in \mathbb{R}^{n}$ and $r>0$. Given a ball $B$, we denote by $c(B)$ its center and by $r(B)$ its radius. In the Euclidean space $\mathbb{R}^{n}$, we write $B(r)$ instead of $B(o, r)$, where $o \equiv(0,0, \ldots, 0)$. 
- A metric measure space is a pair of a metric space $(X, d)$ and a measure $\mu$ such that any open set is measurable.

- By a "cube" we mean a compact cube in $\mathbb{R}^{n}$ whose edges are parallel to the coordinate axes. The metric closed ball defined by $\ell^{\infty}$ is called a cube. If a cube has center $x$ and radius $r$, we denote it by $Q(x, r)$. Namely, we write

$$
Q(x, r) \equiv\left\{y=\left(y_{1}, y_{2}, \ldots, y_{n}\right) \in \mathbb{R}^{n}: \max _{j=1,2, \ldots, n}\left|x_{j}-y_{j}\right| \leq r\right\}
$$

when $x=\left(x_{1}, x_{2}, \ldots, x_{n}\right) \in \mathbb{R}^{n}$ and $r>0$. From the definition of $Q(x, r)$, its volume is $(2 r)^{n}$. We write $Q(r)$ instead of $Q(o, r)$. Given a cube $Q$, we denote by $c(Q)$ the center of $Q$ and by $\ell(Q)$ the sidelength of $Q: \ell(Q)=$ $|Q|^{1 / n}$, where $|Q|$ denotes the volume of the cube $Q$.

- Given a cube $Q$ and $k>0, k Q$ means the cube concentric to $Q$ with sidelength $k \ell(Q)$. Given a ball $B$ and $k>0$, we denote by $k B$ the ball concentric to $B$ with radius $k r(B)$.

- Let $A, B \geq 0$. Then $A \lesssim B$ and $B \gtrsim A$ mean that there exists a constant $C>0$ such that $A \leq \widetilde{C} B$, where $C$ depends only on the parameters of importance. The symbol $A \sim B$ means that $A \lesssim B$ and $B \lesssim A$ happen simultaneously, while $A \simeq B$ means that there exists a constant $C>0$ such that $A=C B$.

- When we need to emphasize or keep in mind that the constant $C$ depends on the parameters $\alpha, \beta, \gamma$ etc Instead of $A \lesssim B$, we write $A \lesssim \alpha, \beta, \gamma, \ldots B$.

\section{Preliminaries}

2.1. Lebesgue spaces and integral inequalities. Here we recall Lebesgue spaces and the integral inequalities.

Definition 2.1 (Lebesgue space). Let $(X, \mathcal{B}, \mu)$ be a measure space and $0<p \leq \infty$.

- The Lebesgue norm $L^{p}(\mu)$-(quasi-)norm of a measurable function $f$ is given by

$$
\begin{aligned}
\|f\|_{p} & \equiv\|f\|_{L^{p}(\mu)} \equiv\left(\int_{X}|f(x)|^{p} d \mu(x)\right)^{\frac{1}{p}}, p<\infty \\
\|f\|_{\infty} & \equiv\|f\|_{L^{\infty}(\mu)} \equiv \sup \{\lambda>0:|f(x)| \leq \lambda \text { for } \mu \text {-a.e. } x \in X\}, p=\infty .
\end{aligned}
$$

- Define

$$
L^{p}(\mu) \equiv\left\{f: X \rightarrow \overline{\mathbb{K}}: f \text { is measurable and }\|f\|_{p}<\infty\right\} / \sim .
$$

Here the equivalence relation $\sim$ is defined by:

$$
f \sim g \Longleftrightarrow f=g \text { a.e. }
$$

and below omit this equivalence in defining function spaces.

As usual if $(X, \mathcal{B}, \mu)$ is the Lebesgue measure, then we write $L^{p}\left(\mathbb{R}^{n}\right)=L^{p}(\mu)$ and $\|\cdot\|_{\mathrm{W} L^{p}}=\|\cdot\|_{\mathrm{W} L^{p}(\mu)}$. 
Theorem 2.2 (Hölder's inequality). Let $0<p, q, r \leq \infty$ satisfy $\frac{1}{r}=\frac{1}{p}+\frac{1}{q}$. Then for $f \in L^{p}(\mu)$ and $g \in L^{q}(\mu),\|f g\|_{L^{r}(\mu)} \leq\|f\|_{L^{p}(\mu)}\|g\|_{L^{q}(\mu)}$. See [1, Theorem 2.4] for example.

In addition to $L^{p}(\mu)$ with $0<p \leq \infty$, it is convenient to define $L^{0}(\mu)$ : The space $L^{0}(\mu)$ denotes the set of all measurable functions considered modulo the difference on the set of measure zero.

2.2. Integral operators. We will study the Hardy-Littlewood maximal operators, the Riesz potentails, the Riesz transforms and the fractional maximal operators. So we recall the definition together with the boundedness properties on $L^{p}\left(\mathbb{R}^{n}\right)$. For Theorems 2.4, 2.5, 2.9, 2.10, 2.11 we refer to $[24,92,116,115]$.

Definition 2.3 (Hardy-Littlewood maximal operator). For $f \in L^{0}\left(\mathbb{R}^{n}\right)$, define a function $M f$ by

$$
M f(x) \equiv \sup _{B \in \mathcal{B}} \frac{\chi_{B}(x)}{|B|} \int_{B}|f(y)| \mathrm{d} y \quad\left(x \in \mathbb{R}^{n}\right) .
$$

The mapping $M: f \mapsto M f$ is called the Hardy-Littlewood maximal operator.

We have the weak- $(1,1)$ boundedness of $M$.

Theorem 2.4 (Hardy-Littlewood maximal inequality). For $f \in L^{1}\left(\mathbb{R}^{n}\right), \lambda>0$,

$$
\lambda|\{M f>\lambda\}| \leq 3^{n}\|f\|_{1} .
$$

For $1<p \leq \infty$ we have the strong- $(p, p)$ boundedness.

Theorem $2.5\left(L^{p}\left(\mathbb{R}^{n}\right)\right.$-inequality $)$. Let $1<p<\infty$. Then

$$
\|M f\|_{p} \leq\left(\frac{p 2^{p} \cdot 3^{n}}{p-1}\right)^{\frac{1}{p}}\|f\|_{p}
$$

for all $f \in L^{0}\left(\mathbb{R}^{n}\right)$.

We move on to the singular integral operators. Among others we consider the Calderón-Zygmund operators.

Here we are interested in the following type of the singular integral operators:

Definition 2.6 (Singular integral operator). A singular integral operator is an $L^{2}\left(\mathbb{R}^{n}\right)$-bounded linear operator $T$ that comes with a function $K \in C^{1}\left(\mathbb{R}^{n} \backslash\{0\}\right)$ satisfying the following conditions:

- (Size condition) For all $x \in \mathbb{R}^{n}$,

$$
|K(x)| \lesssim|x|^{-n} .
$$

- (Gradient condition) For all $x \in \mathbb{R}^{n}$,

$$
|\nabla K(x)| \lesssim|x|^{-n-1} .
$$


- Let $f$ be an $L^{2}\left(\mathbb{R}^{n}\right)$-function. For almost all $x \notin \operatorname{supp}(f)$,

$$
T f(x)=\int_{\mathbb{R}^{n}} K(x-y) f(y) \mathrm{d} y .
$$

The function $K$ is called the integral kernel of $T$.

More generally we consider the following type of singular integral operators:

Definition 2.7. A (generalized) Calderón-Zygmund operator is an $L^{2}\left(\mathbb{R}^{n}\right)$-bounded linear operator $T$, if it satisfies the following conditions:

- There exists a measurable function $K$ such that for all $L^{\infty}\left(\mathbb{R}^{n}\right)$-functions $f$ with compact support we have

$$
T f(x)=\int_{\mathbb{R}^{n}} K(x, y) f(y) d y \text { for all } x \notin \operatorname{supp} f .
$$

- The kernel function $K$ satisfies the following estimates.

$$
|K(x, y)| \lesssim|x-y|^{-n}
$$

if $x \neq y$ and

$$
|K(x, z)-K(y, z)|+|K(z, x)-K(z, y)| \lesssim \frac{|x-y|}{|x-z|^{n+1}},
$$

if $0<2|x-y|<|z-x|$.

As a typical example we consider the Riesz transform:

Example 2.8. Let $j=1,2, \ldots, n$. The singular integral operators, which are represented by the $j$-th Riesz transform given by,

$$
R_{j} f(x) \equiv \lim _{\varepsilon \downarrow 0} \int_{\mathbb{R}^{n} \backslash B(x, \varepsilon)} \frac{x_{j}-y_{j}}{|x-y|^{n+1}} f(y) \mathrm{d} y,
$$

are integral operators with singularity.

We have the weak- $(1,1)$ boundedness as before.

Theorem 2.9. Suppose that $T$ is a CZ-operator. Then $T$ is weak- $(1,1)$ bounded, that is

$$
|\{|T f|>\lambda\}| \lesssim \frac{1}{\lambda} \int_{\mathbb{R}^{n}}|f(x)| d x \quad(\lambda>0)
$$

for all $f \in L^{1}\left(\mathbb{R}^{n}\right) \cap L^{2}\left(\mathbb{R}^{n}\right)$.

We have the strong boundedness for $1<p<\infty$.

Theorem 2.10. Let $1<p<\infty$ and $T$ be a generalized singular integral operator, which is initially defined on $L^{2}\left(\mathbb{R}^{n}\right)$. Then $T$ can be extended to $L^{p}\left(\mathbb{R}^{n}\right)$ for all $1<p<\infty$ so that

$$
\|T f\|_{p} \lesssim p\|f\|_{p}
$$

for all $f \in L^{p}\left(\mathbb{R}^{n}\right)$. 
As a different type of singular integral operators, we consider the fractional integral operators. Let $0<\alpha<n$. Let $I_{\alpha}$ be the fractional maximal operator given by

$$
I_{\alpha} f(x) \equiv \int_{\mathbb{R}^{n}} \frac{f(y)}{|x-y|^{n-\alpha}} \mathrm{d} y \quad\left(x \in \mathbb{R}^{n}\right)
$$

for a nonnegative measurable function $f: \mathbb{R}^{n} \rightarrow[0, \infty]$.

The following theorem is known as the Hardy-Littlewood-Sobolev theorem. Generalized Morrey spaces can be used to refine this theorem.

Theorem 2.11 (Hardy-Littlewood-Sobolev). [54, 55, 111] Let $0<\alpha<n$.

- We have

$$
\lambda^{\frac{n}{n-\alpha}}\left|\left\{x \in \mathbb{R}^{n}:\left|I_{\alpha} f(x)\right|>\lambda\right\}\right| \lesssim\|f\|_{1}
$$

for all $f \in L^{1}\left(\mathbb{R}^{n}\right)$ and $\lambda>0$.

- Assume that the parameters $1<p<\frac{n}{\alpha}$ and $\frac{1}{q}=\frac{1}{p}-\frac{\alpha}{n}$. Then we have $\left\|I_{\alpha} f\right\|_{q} \lesssim\|f\|_{p}$ for all $f \in L^{p}\left(\mathbb{R}^{n}\right)$.

Let $0 \leq \alpha<n$. The fractional maximal operator $M_{\alpha}$ of order $\alpha$ is defined by

$$
M_{\alpha} f(x) \equiv \sup _{Q \in \mathcal{Q}} \ell(Q)^{\alpha-n} \chi_{Q}(x) \int_{Q}|f(y)| d y
$$

for a measurable function $f$. Note that $M_{\alpha}$ maps $\mathcal{M}_{1}^{n / \alpha}\left(\mathbb{R}^{n}\right)$ boundedly to $L^{\infty}\left(\mathbb{R}^{n}\right)$. As an opposite endpoint case, we have the following boundedness:

Theorem 2.12. Let $0<\alpha<n$. Then

$$
\lambda^{\frac{n}{n-\alpha}}\left|\left\{x \in \mathbb{R}^{n}: M_{\alpha} f(x)>\lambda\right\}\right| \lesssim\|f\|_{1}
$$

for all $f \in L^{1}\left(\mathbb{R}^{n}\right)$.

Using the boundedness of $M$, we can prove the boundedness of $M_{\alpha}$. Here for the sake of convenience for readers we supply the proof.

Theorem 2.13. Let $1<p<q<\infty$ and $0<\alpha<n$ satisfy $\frac{1}{q}=\frac{1}{p}-\frac{\alpha}{n}$. Then $\left\|M_{\alpha} f\right\|_{L^{q}} \lesssim\|f\|_{L^{p}}$ for all $f \in L^{p}\left(\mathbb{R}^{n}\right)$.

Proof. By the monotone convergence theorem we may assume that $f \in L_{\mathrm{c}}^{\infty}\left(\mathbb{R}^{n}\right)$. For $\lambda>0$ we let $\Omega_{\lambda} \equiv\left\{M_{\alpha} f>\lambda\right\}$. We observe that

$$
\left(\left\|M_{\alpha} f\right\|_{L^{q}}\right)^{q}=q \int_{0}^{\infty} \lambda^{q-1}\left|\Omega_{\lambda}\right| d \lambda \leq q \int_{0}^{\infty} \lambda^{q-1-\frac{n}{n-\alpha}}\left(\left\|f \chi_{\Omega_{\lambda}}\right\|_{L^{1}}\right)^{\frac{n}{n-\alpha}} d \lambda .
$$

We choose $u>0$ so that $n(1-u)=p \alpha$. Then we have

$$
\left\|f \chi_{\Omega_{\lambda}}\right\|_{L^{1}} \leq\left(\left\|f \chi_{\Omega_{\lambda}}\right\|_{L^{\frac{n u}{n-\alpha}}}\right)^{u}\left(\|f\|_{L^{p}}\right)^{1-u} .
$$


Thus, we have

$$
\begin{aligned}
\left(\left\|M_{\alpha} f\right\|_{L^{q}}\right)^{q} & \lesssim \int_{0}^{\infty} \lambda^{q-1-\frac{n}{n-\alpha}}\left(\left\|f \chi_{\Omega_{\lambda}}\right\|_{L^{\frac{n u}{n-\alpha}}}\right)^{\frac{n u}{n-\alpha}}\left(\|f\|_{L^{p}}\right)^{\frac{n(1-u)}{n-\alpha}} d \lambda \\
& \simeq\left(\|f\|_{L^{p}}\right)^{\frac{n(1-u)}{n-\alpha}} \int_{\mathbb{R}^{n}}|f(x)|^{\frac{n u}{n-\alpha}} M_{\alpha} f(x)^{q-\frac{n}{n-\alpha}} d x .
\end{aligned}
$$

Observe that

$$
\left(q-\frac{n}{n-\alpha}\right) \times\left(\frac{(n-\alpha) p}{n u}\right)^{\prime}=\frac{q(p-1) n}{(p-1) n} .
$$

By the Hölder inequality, we obtain

$$
\int_{\mathbb{R}^{n}}|f(x)|^{\frac{n u}{n-\alpha}} M_{\alpha} f(x)^{q-\frac{n}{n-\alpha}} d \mu(x) \leq\left(\|f\|_{L^{p}}\right)^{\frac{n u}{n-\alpha}}\left(\left\|M_{\alpha} f\right\|_{L^{q}}\right)^{q-\frac{n}{n-\alpha}} .
$$

As a result,

$$
\left(\left\|M_{\alpha} f\right\|_{L^{q}}\right)^{q} \lesssim\left(\|f\|_{L^{p}}\right)^{\frac{n}{n-\alpha}}\left(\left\|M_{\alpha} f\right\|_{L^{q}}\right)^{q-\frac{n}{n-\alpha}} .
$$

If we arrange this inequality, we obtain

$$
\left\|M_{\alpha} f\right\|_{L^{q}} \lesssim\|f\|_{L^{p}}
$$

In addition to the linear operators above we sometimes consider the commutator generated by BMO and these operators. Here we recall the BMO (bounded mean oscillation) as follows:

Definition 2.14 $\left(\mathrm{BMO}\left(\mathbb{R}^{n}\right)\right.$ (space)). Define

$$
\|f\|_{\mathrm{BMO}} \equiv \sup _{Q \in \mathcal{Q}} \frac{1}{|Q|} \int_{Q}\left|f(y)-m_{Q}(f)\right| \mathrm{d} y=\sup _{Q \in \mathcal{Q}} m_{Q}\left(\left|f-m_{Q}(f)\right|\right)
$$

for $f \in L_{\text {loc }}^{1}\left(\mathbb{R}^{n}\right)$. The "norm" $\|\cdot\|_{\mathrm{BMO}}$ is called the $\mathrm{BMO}\left(\mathbb{R}^{n}\right)$ norm. The space $\mathrm{BMO}\left(\mathbb{R}^{n}\right)$ collects all $f \in L_{\text {loc }}^{1}\left(\mathbb{R}^{n}\right)$ such that $\|f\|_{\mathrm{BMO}}$ is finite. Usually $\mathrm{BMO}\left(\mathbb{R}^{n}\right)$ is considered modulo the constant functions.

As the following theorem shows, the BMO functions have high local integrability.

Theorem 2.15 (John-Nirenberg inequality). For any $\lambda>0$, a cube $Q$ and $a$ nonconstant $\mathrm{BMO}\left(\mathbb{R}^{n}\right)$-function $b$,

where $D$ depends only on the dimension.

$$
\mid\left\{x \in Q:\left|b(x)-m_{Q}(b)\right|>\lambda\right\} \| \lesssim_{n} \exp \left(-\frac{D \lambda}{\|b\|_{\mathrm{BMO}}}\right),
$$

We will consider the commutators generated by BMO and these operators. For the properties of these commutators we refer to [25]. Let $a \in \operatorname{BMO}\left(\mathbb{R}^{n}\right)$. Then the we can consider the operators

$$
f \in L_{\mathrm{c}}^{\infty}\left(\mathbb{R}^{n}\right) \mapsto[a, T] f=a \cdot T f-T[a \cdot f], \quad\left[a, I_{\alpha}\right] f=a \cdot I_{\alpha} f-I_{\alpha}[a \cdot f]
$$

despite the ambiguity which the definition of $\operatorname{BMO}\left(\mathbb{R}^{n}\right)$ causes. 
Thanks to the following theorem, we can extend our results of generalized Morrey spaces by mimicking the proof of the boundedness for the similar operators. However, due to the similarity we do not consider the boundedness and the definition of these operators. Instead, we give some references when needed.

Theorem 2.16. Let $0<\alpha<n, a \in \mathrm{BMO}\left(\mathbb{R}^{n}\right)$ and $T$ be a generalized CalderónZygmund operator.

- Let $1<p<\infty$. We have $\|[a, T] f\|_{L^{p}} \lesssim\|a\|_{\text {BMO }}\|f\|_{L^{p}}$ for all $f \in L_{\mathrm{c}}^{\infty}\left(\mathbb{R}^{n}\right)$.

- Let $1<p<q<\infty$ satisfy $\frac{1}{q}=\frac{1}{p}-\frac{\alpha}{n}$. Then we have $\left\|\left[a, I_{\alpha}\right] f\right\|_{L^{q}} \lesssim$ $\|a\|_{\mathrm{BMO}}\|f\|_{L^{p}}$ for all $f \in L_{\mathrm{c}}^{\infty}\left(\mathbb{R}^{n}\right)$.

For the proof of Theorem 2.16 we refer to [25].

\section{Generalized Morrey spaces}

Likewise we can replace $q$ by a function $\Phi$.

3.1. Definition of generalized Morrey spaces. From the observation above, we are led to the following definition:

Definition 3.1. Let $0<q<\infty$ and $\varphi:(0, \infty) \rightarrow[0, \infty)$ be a function which does not satisfy $\varphi \equiv 0$.

- [73] Define the generalized Morrey space $\mathcal{M}_{q}^{\varphi}\left(\mathbb{R}^{n}\right)$ to be the set of all measurable functions $f$ such that

$$
\|f\|_{\mathcal{M}_{q}^{\varphi}} \equiv \sup _{x \in \mathbb{R}^{n}, r>0} \varphi(r)\left(\frac{1}{|Q(x, r)|} \int_{Q(x, r)}|f(y)|^{q} d y\right)^{\frac{1}{q}}<\infty .
$$

- [28] Define the weak generalized Morrey space $\mathrm{w} \mathcal{M}_{q}^{\varphi}\left(\mathbb{R}^{n}\right)$ to be the set of all measurable functions $f$ such that

$$
\|f\|_{\mathrm{w} \mathcal{M}_{q}^{\varphi}} \equiv \sup _{\lambda>0}\left\|\lambda \chi_{(\lambda, \infty)}(|f|)\right\|_{\mathcal{M}_{q}^{\varphi}}<\infty .
$$

Although we torelate the case where $\varphi(t)=0$ for some $t>0$, it turns out that there is no need to consider such possibility.

Before we go into more details, clarifying remarks may be in order.

Remark 3.2. In [73] Nakai defined the generalized Morrey space $\mathcal{M}_{q}^{\varphi}\left(\mathbb{R}^{n}\right)$ to be the set of all measurable functions $f$ such that

$$
\|f\|_{\mathcal{M}_{q}^{\varphi}} \equiv \sup _{x \in \mathbb{R}^{n}, r>0} \frac{1}{\varphi(r)}\left(\frac{1}{|Q(x, r)|} \int_{Q(x, r)}|f(y)|^{q} d y\right)^{\frac{1}{q}}<\infty,
$$

or more generally

$$
\|f\|_{\mathcal{M}_{q}^{\varphi}} \equiv \sup _{x \in \mathbb{R}^{n}, r>0} \frac{1}{\varphi(x, r)}\left(\frac{1}{|Q(x, r)|} \int_{Q(x, r)}|f(y)|^{q} d y\right)^{\frac{1}{q}}<\infty .
$$


See also $[19,29,33,60,76,120,123]$. Here we follow the notation by Sawano, Sugano and Tanaka [100, 101, 102], for example. See also [71]. See [3, 12, 86, 88, 89, 109] for another notation of generalized Morrey spaces, for example. Despite the difference of the notation, the idea of defining the predual space depends on $[60,109]$.

Remark 3.3. Some prefer to call $\varphi$ the weight (see [26] for example), while some prefer to call $\|\cdot \times w\|_{\mathcal{M}_{q}^{\varphi}}$ the weighted generalized Morrey norm (see [41] for example).

We can recover the Lebesgue space $L^{q}\left(\mathbb{R}^{n}\right)$ by letting $\varphi(t) \equiv t^{\frac{n}{q}}$ for $t>0$ as we have mentioned. To compare Morrey spaces with generalized Morrey spaces, we sometimes call Morrey spaces classical Morrey spaces. In addition to the function $\varphi(t)=t^{\frac{n}{p}}$, we consider the following typical functions:

\section{Example 3.4.}

- The function $\varphi \equiv 1$ generates $L^{\infty}\left(\mathbb{R}^{n}\right)$ thanks to the Lebesgue convergence theorem.

- Let $0<q<\infty$ and $a \in \mathbb{R}$. Define $\varphi(t)=t^{\frac{n}{q}}(\log (e+t))^{a}$ for $t>0$. We remark that $\mathcal{M}_{q}^{\varphi}\left(\mathbb{R}^{n}\right) \neq\{0\}$ if and only if $a \leq 0$. In fact, we have

$$
\|f\|_{\mathcal{M}_{q}^{\varphi}}=\sup _{x \in \mathbb{R}^{n}, r>0}(\log (e+r))^{a}\|f\|_{L^{q}(Q(x, r))} .
$$

Thus, if $f$ is a measurable function such that $\|f\|_{\mathcal{M}_{q}^{\varphi}}<\infty$ and if $a>0$, then we have $\|f\|_{L^{q}(Q(x, r))}=0$ for any cube $Q(x, r)$. Thus, $f=0$ a.e.. Convesely if $a \leq 0$, then $L^{q}\left(\mathbb{R}^{n}\right) \subset \mathcal{M}_{q}^{\varphi}\left(\mathbb{R}^{n}\right)$.

- Let $0<q \leq p_{1}<p_{2}<\infty$. Then $\varphi(t)=t^{\frac{n}{p_{1}}}+t^{\frac{n}{p_{2}}}, t>0$ can be used to express the intersection of $\mathcal{M}_{q}^{p_{1}}\left(\mathbb{R}^{n}\right) \cap \mathcal{M}_{q}^{p_{2}}\left(\mathbb{R}^{n}\right)$. In general, for $0<q<\infty$ and $\varphi_{1}, \varphi_{2}:(0, \infty) \rightarrow[0, \infty)$ satisfying $\varphi_{1}\left(t_{1}\right) \neq 0$ and $\varphi\left(t_{2}\right) \neq 0$ for some $t_{1}, t_{2}>0 \mathcal{M}_{q}^{\varphi_{1}}\left(\mathbb{R}^{n}\right) \cap \mathcal{M}_{q}^{\varphi_{2}}\left(\mathbb{R}^{n}\right)=\mathcal{M}_{q}^{\varphi_{1}+\varphi_{2}}\left(\mathbb{R}^{n}\right)$ with equivalence of norms.

- Let $0<q \leq p_{1}<p_{2}<\infty$. Let $\varphi(t)=\chi_{\mathbb{Q} \cap(0, \infty)}(t) t^{\frac{n}{p_{1}}}+\chi_{(0, \infty) \backslash \mathbb{Q}}(t) t^{\frac{n}{p_{2}}}$ for $t>0$. Then we have $\mathcal{M}_{q}^{\varphi}\left(\mathbb{R}^{n}\right)=\mathcal{M}_{q}^{p_{1}}\left(\mathbb{R}^{n}\right) \cap \mathcal{M}_{q}^{p_{2}}\left(\mathbb{R}^{n}\right)$ and for any $f \in L^{0}\left(\mathbb{R}^{n}\right)$

$$
\|f\|_{\mathcal{M}_{q}^{\varphi}}=\max \left\{\|f\|_{\mathcal{M}_{q}^{p_{1}}},\|f\|_{\mathcal{M}_{q}^{p_{2}}}\right\} .
$$

- We can consider the norm

$$
\sup _{x \in \mathbb{R}^{n}, r \in(0,1)}|Q(x, r)|^{\frac{1}{p}}\left(\frac{1}{|Q(x, r)|} \int_{Q(x, r)}|f(y)|^{q} d y\right)^{\frac{1}{q}}
$$

for $0<q<p<\infty$. In fact, we take $\varphi(t)=t^{\frac{n}{p}} \chi_{[0,1]}(t)$ for $t>0$.

- Likewise we can consider the norm

$$
\sup _{x \in \mathbb{R}^{n}, r \in[1, \infty)}|Q(x, r)|^{\frac{1}{p}}\left(\frac{1}{|Q(x, r)|} \int_{Q(x, r)}|f(y)|^{q} d y\right)^{\frac{1}{q}}
$$

for $0<q<p<\infty$. In fact, we take $\varphi(t)=t^{\frac{n}{p}} \chi_{[1, \infty)}(t)$ for $t>0$. 
- Let $0<q<\infty$ The uniformly locally $L^{q}$-integrable space $L_{\mathrm{uloc}}^{q}\left(\mathbb{R}^{n}\right)$ is the set of all measurable functions $f$ for which

$$
\sup _{x \in \mathbb{R}^{n}, r \in(0,1)}\left(\int_{Q(x, r)}|f(y)|^{q} d y\right)^{\frac{1}{q}}=\sup _{x \in \mathbb{R}^{n}}\left(\int_{Q(x, 1)}|f(y)|^{q} d y\right)^{\frac{1}{q}}
$$

is finite. As before, if we let $\varphi(t)=t^{\frac{n}{q}} \chi_{(0,1]}(t)$ for $t>0$, then we obtain $L_{\mathrm{uloc}}^{q}\left(\mathbb{R}^{n}\right)=\mathcal{M}_{q}^{\varphi}\left(\mathbb{R}^{n}\right)$. We can define the weak uniformly locally $L^{q}$ integrable space $\mathrm{w} L_{\mathrm{uloc}}^{q}\left(\mathbb{R}^{n}\right)$ similarly. For $f \in L^{0}\left(\mathbb{R}^{n}\right)$, the norm is given by

$$
\|f\|_{\mathrm{w} L_{\mathrm{uloc}}^{q}} \equiv \sup _{\lambda>0} \lambda\left\|\chi_{(\lambda, \infty]}(|f|)\right\|_{L_{\mathrm{uloc}}^{q}} .
$$
follows:

The fifth example deserves a name. We define the small Morrey space as

Definition 3.5. Let $0<q<p<\infty$. The small Morrey space $m_{q}^{p}\left(\mathbb{R}^{n}\right)$ is the set of all measurable functions $f$ for which the quantity

$$
\|f\|_{m_{q}^{p}} \equiv \sup _{x \in \mathbb{R}^{n}, r \in(0,1)}|Q(x, r)|^{\frac{1}{p}}\left(\frac{1}{|Q(x, r)|} \int_{Q(x, r)}|f(y)|^{q} d y\right)^{\frac{1}{q}}
$$

is finite. The weak small Morrey space $\mathrm{w} m_{q}^{p}\left(\mathbb{R}^{n}\right)$ is defined similarly. For $f \in$ $L^{0}\left(\mathbb{R}^{n}\right)$, the norm is given by

$$
\|f\|_{\mathrm{w} m_{q}^{p}} \equiv \sup _{\lambda>0} \lambda\left\|\chi_{(\lambda, \infty]}(|f|)\right\|_{m_{q}^{p}} .
$$

Example 3.6. [14, Proposition A] Let $x \in \mathbb{R}^{n}$ and $r>0$. Then

$$
\left\|\chi_{Q(x, r)}\right\|_{\mathcal{M}_{q}^{\varphi}}=\sup _{t>0} \varphi(t) \min \left(t^{-\frac{n}{q}}, r^{-\frac{n}{q}}\right) .
$$

In fact, simply observe that

$$
\begin{aligned}
\left\|\chi_{Q(x, r)}\right\|_{\mathcal{M}_{q}^{\varphi}} & \equiv \sup _{R>0} \varphi(R)\left(\frac{|Q(x, R) \cap Q(x, r)|}{|Q(x, R)|}\right)^{\frac{1}{q}} \\
& =\sup _{t>0} \varphi(t) \min \left(t^{-\frac{n}{q}}, r^{-\frac{n}{q}}\right) .
\end{aligned}
$$

The following $\min (1, q)$-triangle inequality holds:

Lemma 3.7. Let $0<q<\infty$ and $\varphi:(0, \infty) \rightarrow(0, \infty)$ be a function. Then

$$
\|f+g\|_{\mathcal{M}_{q}^{\varphi}}^{\min (1, q)} \leq\|f\|_{\mathcal{M}_{q}^{\varphi}}^{\min (1, q)}+\|g\|_{\mathcal{M}_{q}^{\varphi}} \min (1, q)
$$

for all $f, g \in \mathcal{M}_{q}^{\varphi}\left(\mathbb{R}^{n}\right)$.

Proof. This is similar to classical Morrey spaces: Use the $\min (1, q)$-triangle inequality for the Lebesgue space $L^{q}\left(\mathbb{R}^{n}\right)$.

Proposition 3.8. Let $0<q<\infty$, and let $\varphi:(0, \infty) \rightarrow[0, \infty)$ be a function satisfying $\varphi\left(t_{0}\right) \neq 0$ for some $t_{0}>0$. Then $\mathcal{M}_{q}^{\varphi}\left(\mathbb{R}^{n}\right)$ is a quasi-Banach space and if $q \geq 1$, then $\mathcal{M}_{q}^{\varphi}\left(\mathbb{R}^{n}\right)$ is a Banach space. 
Proof. The norm inequality follows from Lemma 3.7. The proof of the completeness is a routine, which we omit.

Proposition 3.8 guarantees that the (quasi-)norm of $\mathcal{M}_{q}^{\varphi}\left(\mathbb{R}^{n}\right)$ is complete. However, it may happen that $\mathcal{M}_{q}^{\varphi}\left(\mathbb{R}^{n}\right)=\{0\}$ as is seen from Example 3.10. We check that this extraordinary thing never happens if $\varphi$ satisfies a mild condition.

Proposition 3.9. [80, Lemma 2.2(2)] Let $0<q<\infty$, and let $\varphi:(0, \infty) \rightarrow$ $[0, \infty)$ be a function satisfying $\varphi\left(t_{0}\right) \neq 0$ for some $t_{0}>0$. Then the following are equivalent:

(a) $L_{\mathrm{c}}^{\infty}\left(\mathbb{R}^{n}\right) \subset \mathcal{M}_{q}^{\varphi}\left(\mathbb{R}^{n}\right)$.

(b) $\mathcal{M}_{q}^{\varphi}\left(\mathbb{R}^{n}\right) \neq\{0\}$.

(c) $\sup _{t>0} \varphi(t) \min \left(t^{-\frac{n}{q}}, 1\right)<\infty$.

Proof. It is clear that $(a)$ implies $(b)$.

Assume $(b)$. Then there exists $f \in \mathcal{M}_{q}^{\varphi}\left(\mathbb{R}^{n}\right) \backslash\{0\}$. We may assume that $f(0) \neq 0$ and that $x=0$ is the Lebesgue point of $|f|^{q}$. Then since $f \in L_{\text {loc }}^{q}\left(\mathbb{R}^{n}\right)$, by the Lebesgue differential theorem,

$$
\frac{1}{|Q(r)|} \int_{Q(r)}|f(y)|^{q} d y \sim 1
$$

for all $0<r<1$. Here the implicit constants depend on $f$. Thus,

$$
\sup _{0<t \leq 1} \varphi(t) \sim \sup _{0<t \leq 1} \varphi(t)\left(\frac{1}{|Q(t)|} \int_{Q(t)}|f(y)|^{q} d y\right)^{\frac{1}{q}} \leq\|f\|_{\mathcal{M}_{q}^{\varphi}}<\infty .
$$

Here the implicit constants depend on $f$ again. Meanwhile

$$
\begin{aligned}
\sup _{t \geq 1} t^{-\frac{n}{q}} \varphi(t) & \lesssim \sup _{t \geq 1} \varphi(t)\left(\frac{1}{|Q((t+1,0,0, \ldots, 0), t)|} \int_{Q((t+1,0,0, \ldots, 0), t)}|f(y)|^{q} d y\right)^{\frac{1}{q}} \\
& \leq\|f\|_{\mathcal{M}_{q}^{\varphi}}<\infty .
\end{aligned}
$$

Thus we conclude $(c)$.

Finally, if $(c)$ holds, then $\chi_{Q(x, 1)} \in \mathcal{M}_{q}^{\varphi}\left(\mathbb{R}^{n}\right)$ for any $x \in \mathbb{R}^{n}$. Since $\mathcal{M}_{q}^{\varphi}\left(\mathbb{R}^{n}\right)$ is a linear space, $\chi_{Q(x, m)} \in \mathcal{M}_{q}^{\varphi}\left(\mathbb{R}^{n}\right)$ for any $x \in \mathbb{R}^{n}$. Since any function $g \in L_{\mathrm{c}}^{\infty}\left(\mathbb{R}^{n}\right)$ admits the estimate of the form $|g| \leq N \chi_{Q(x, m)}$ for some $m, N \in \mathbb{N}$, we conclude $(a)$.

Here we consider the case where $\mathcal{M}_{q}^{\varphi}\left(\mathbb{R}^{n}\right)$ is close to $\mathcal{M}_{q}^{p}\left(\mathbb{R}^{n}\right)$ in a certain sense.

Example 3.10. Let $0<q \leq p<\infty$ and $\mathbb{B}=\left(\beta_{1}, \beta_{2}\right) \in \mathbb{R}^{2}$. We write

$$
\ell^{\mathbb{B}}(r)=\ell^{\left(\beta_{1}, \beta_{2}\right)}(r) \equiv \begin{cases}(1+|\log r|)^{\beta_{1}} & (0<r \leq 1), \\ (1+|\log r|)^{\beta_{2}} & (1<r<\infty) .\end{cases}
$$

Set $-\mathbb{B}=\left(-\beta_{1},-\beta_{2}\right)$. 
- We set $\varphi(t) \equiv t^{\frac{n}{q}} \ell^{-\mathbb{B}}(t), t>0$. Note that $\mathcal{M}_{q}^{\varphi}\left(\mathbb{R}^{n}\right) \neq\{0\}$ if and only if $\beta_{2} \geq$ 0 . Indeed, according to Proposition 3.9 the case $\beta_{2}<0$ must be excluded in order that $\mathcal{M}_{q}^{\varphi}\left(\mathbb{R}^{n}\right) \neq\{0\}$. Convesely if $\beta_{2} \geq 0$, then $L_{\mathrm{c}}^{\infty}\left(\mathbb{R}^{n}\right) \subset \mathcal{M}_{q}^{\varphi}\left(\mathbb{R}^{n}\right)$.

- Let $0<q<p<\infty$. We set $\varphi(t) \equiv t^{\frac{n}{p}} \ell^{-\mathbb{B}}(t), t>0$. Then $L_{\mathrm{c}}^{\infty}\left(\mathbb{R}^{n}\right) \subset$ $\mathcal{M}_{q}^{\varphi}\left(\mathbb{R}^{n}\right)$.

- We set $\varphi(t) \equiv \ell^{-\mathbb{B}}(t), t>0$. For $a \in \mathbb{R}$, we set $f(x) \equiv(1+|x|)^{-a}, x \in \mathbb{R}^{n}$. Let us see that $\mathcal{M}_{q}^{\varphi}\left(\mathbb{R}^{n}\right)$ is close to $L^{\infty}\left(\mathbb{R}^{n}\right)$ if $\beta_{1} \geq 0$.

- By the Lebesgue differentiation theorem, $\|f\|_{\infty} \leq 0$ if $\beta_{1}<0$, so that $f=0$ a.e. So, if $\beta_{1}<0$, then $\mathcal{M}_{q}^{\varphi}\left(\mathbb{R}^{n}\right)=\{0\}$.

- Let $\beta_{1} \geq 0>\beta_{2}$. Then $f \in \mathcal{M}_{q}^{\varphi}\left(\mathbb{R}^{n}\right)$ if and only if $a<0$.

- Let $\beta_{1}, \beta_{2} \geq 0$. Then $f \in \mathcal{M}_{q}^{\varphi}\left(\mathbb{R}^{n}\right)$ if and only if $a \leq 0$.

We have the following scaling law:

Lemma 3.11. [80, Lemma 2.5] Let $0<\eta, q<\infty$ and $\varphi:(0, \infty) \rightarrow(0, \infty)$ be a function. Then $f \in \mathcal{M}_{q}^{\varphi}\left(\mathbb{R}^{n}\right)$ if and only if $|f|^{\eta} \in \mathcal{M}_{q / \eta}^{\varphi^{\eta}}\left(\mathbb{R}^{n}\right)$. Furthemore in this case $\left\||f|^{\eta}\right\|_{\mathcal{M}_{q / \eta}^{\varphi^{\eta}}}=\|f\|_{\mathcal{M}_{q}^{\varphi^{\eta}}}$.

Proof. We content ourselves with showing the equality $\left\||f|^{\eta}\right\|_{\mathcal{M}_{q / \eta}^{\varphi^{\eta}}}=\|f\|_{\mathcal{M}_{q}^{\varphi}}$. We calculate

$$
\left\||f|^{\eta}\right\|_{\mathcal{M}_{q / \eta}^{\varphi^{\eta}}}=\sup _{Q=Q(a, r)} \frac{\varphi(r)^{\eta}\|f\|_{L^{q}(Q)^{\eta}}^{\eta}}{|Q|^{\frac{\eta}{q}}}=\|f\|_{\mathcal{M}_{q}^{\varphi}}{ }^{\eta}
$$

by using $\left\||f|^{\eta}\right\|_{\frac{q}{\eta}}=\|f\|_{q^{\eta}}$.

The nesting property holds like classical Morrey spaces.

Lemma 3.12. Let $0<q_{1} \leq q_{2}<\infty$ and $\varphi:(0, \infty) \rightarrow(0, \infty)$ be a function. Then $\mathcal{M}_{q_{2}}^{\varphi}\left(\mathbb{R}^{n}\right) \subset \mathcal{M}_{q_{1}}^{\varphi}\left(\mathbb{R}^{n}\right)$.

Proof. The proof of Lemma 3.12 hinges on the Hölder inequality. Let $f \in L^{0}\left(\mathbb{R}^{n}\right)$. We write out the norms fully:

$$
\begin{aligned}
\|f\|_{\mathcal{M}_{q_{1}}^{p}} & \equiv \sup _{x \in \mathbb{R}^{n}, r>0} \varphi(r)\left(\frac{1}{|B(x, r)|} \int_{B(x, r)}|f(y)|^{q_{1}} d y\right)^{\frac{1}{q_{1}}} \\
\|f\|_{\mathcal{M}_{q_{0}}^{p}} & =\sup _{x \in \mathbb{R}^{n}, r>0} \varphi(r)\left(\frac{1}{|B(x, r)|} \int_{B(x, r)}|f(y)|^{q_{0}} d y\right)^{\frac{1}{q_{0}}}
\end{aligned}
$$

By the Hölder inequality (for probability measures), we have

$$
\left(\frac{1}{|B(x, r)|} \int_{B(x, r)}|f(y)|^{q_{1}} d y\right)^{\frac{1}{q_{1}}} \leq\left(\frac{1}{|B(x, r)|} \int_{B(x, r)}|f(y)|^{q_{0}} d y\right)^{\frac{1}{q_{0}}} .
$$

Thus inserting inequality (19) into (17) and (18), we obtain $\mathcal{M}_{q_{2}}^{\varphi}\left(\mathbb{R}^{n}\right) \subset \mathcal{M}_{q_{1}}^{\varphi}\left(\mathbb{R}^{n}\right)$. 
3.2. The class $\mathcal{G}_{q}$. It will turn out demanding to consider all possible functions $\varphi$. We will single out good functions. We answer the question of what functions are good.

Definition 3.13. An increasing function $\varphi:(0, \infty) \rightarrow(0, \infty)$ is said to belong to the class $\mathcal{G}_{q}$ if $t^{-\frac{n}{q}} \varphi(t) \geq s^{-\frac{n}{q}} \varphi(s)$ for all $0<t \leq s<\infty$.

Remark that $\mathcal{G}_{q_{1}} \subset \mathcal{G}_{q_{2}}$ if $0<q_{2}<q_{1}<\infty$.

Here we list a series of the functions in $\mathcal{G}_{q}$.

Example 3.14. Let $0<q<\infty$.

- Let $u \in \mathbb{R}$, and let $\varphi(t)=t^{u}$ for $t>0$. Then $\varphi$ belongs to $\mathcal{G}_{q}$ if and only if $0 \leq u \leq \frac{n}{q}$.

- Let $0<u \leq \frac{n}{q}, L \gg 1$ and let $\varphi(t)=\frac{t^{u}}{\log (L+t)}$ for $t>0$. Then $\varphi$ belongs to $\mathcal{G}_{q}$.

- If $\varphi_{1}, \varphi_{2} \in \mathcal{G}_{q}$, then $\varphi_{1}+\varphi_{2}, \max \left(\varphi_{1}, \varphi_{2}\right), \min \left(\varphi_{1}, \varphi_{2}\right) \in \mathcal{G}_{q}$.

- Let $0 \leq u \ll 1$, and let $\varphi(t)=\frac{t^{u}}{\log (e+t)}$ for $t \geq 0$. Then $\varphi \notin \mathcal{G}_{q}$ because $\varphi$ is not increasing. property:

We start with a simple observation that any function in $\mathcal{G}_{q}$ enjoys the doubling

Proposition 3.15. If $\varphi \in \mathcal{G}_{q}$ with $0<q<\infty$, then $\varphi(r) \leq \varphi(2 r) \leq 2^{\frac{n}{q}} \varphi(r)$ for all $r>0$.

Proof. The left inequality is a consequence of the fact that $\varphi$ is increasing, while the right inequality follows from the fact that $t \mapsto t^{-\frac{n}{q}} \varphi(t)$ is decreasing.

The next proposition justifies that we can naturally use the class $\mathcal{G}_{q}$.

Proposition 3.16. [74, p. 446] Let $0<q<\infty$ be fixed. Then for any function $\varphi:(0, \infty) \rightarrow[0, \infty)$ satisfying $0<\sup _{t>0} \varphi(t) \min \left(t^{-\frac{n}{q}}, 1\right)<\infty$, there exists a function $\varphi^{*}:(0, \infty) \rightarrow(0, \infty) \in \mathcal{G}_{q}$ such that $\mathcal{M}_{q}^{\varphi}\left(\mathbb{R}^{n}\right)=\mathcal{M}_{q}^{\varphi^{*}}\left(\mathbb{R}^{n}\right)$ with equivalence of norms.

Proof. Let $f$ be a measurable function.

We claim that

$$
\frac{1}{|Q|} \int_{Q}|f(x)|^{q} d x \leq 2^{n} \sup _{Q^{\prime} \in \mathcal{Q}: Q^{\prime} \subset Q, \ell\left(Q^{\prime}\right)=t^{\prime}} \frac{1}{\left|Q^{\prime}\right|} \int_{Q^{\prime}}|f(x)|^{q} d x
$$

for any cube $Q \in \mathcal{Q}$ and any positive number $t^{\prime} \leq \ell(Q)$. In fact, let

$$
N=\left[1+\frac{\ell(Q)}{t^{\prime}}\right] \in[1, \infty)
$$


We write $Q=\prod_{j=1}^{n}\left[a_{j}, a_{j}+\ell(Q)\right]$, so that $\left(a_{1}, a_{2}, \ldots, a_{n}\right)$ is the "bottom" corner of Q. Define

$$
\delta_{m_{j}} \equiv \begin{cases}0 & \left(m_{j}<N\right) \\ N t^{\prime}-\ell(Q) & \left(m_{j}=N\right)\end{cases}
$$

for $m_{j} \in\{1,2, \ldots, N\}$ and

$$
Q_{m} \equiv \prod_{j=1}^{n}\left[a_{j}+\left(m_{j}-1\right) t^{\prime}-\delta_{m_{j}}, a_{j}+m_{j} t^{\prime}-\delta_{m_{j}}\right]
$$

for $m=\left(m_{1}, m_{2}, \ldots, m_{n}\right) \in\{1,2, \ldots, N\}^{n}$. Then

$$
1 \leq \sum_{m \in\{1,2, \ldots, N\}^{n}} \chi_{Q_{m}} \leq 2^{n}
$$

almost everywhere. As a consequence,

$$
\begin{aligned}
\frac{1}{|Q|} \int_{Q}|f(x)|^{q} d x & \leq \sum_{m \in\{1,2, \ldots, N\}^{n}} \frac{1}{|Q|} \int_{Q_{m}}|f(x)|^{q} d x \\
& \leq \sum_{m \in\{1,2, \ldots, N\}^{n}} \frac{t^{\prime n}}{|Q|} \frac{1}{\left|Q_{m}\right|} \int_{Q_{m}}|f(x)|^{q} d x .
\end{aligned}
$$

Since

$$
\frac{t^{\prime} N}{\ell(Q)}=\frac{t^{\prime}}{\ell(Q)}\left[1+\frac{\ell(Q)}{t^{\prime}}\right] \leq \frac{t^{\prime}}{\ell(Q)}\left(1+\frac{\ell(Q)}{t^{\prime}}\right) \leq \frac{t^{\prime}}{\ell(Q)}+1 \leq 2
$$

it follows that

$$
\frac{1}{|Q|} \int_{Q}|f(x)|^{q} d x \leq 2^{n} \max _{m \in\{1,2, \ldots, N\}^{n}} \frac{1}{\left|Q_{m}\right|} \int_{Q_{m}}|f(x)|^{q} d x
$$

Thus, (20) follows.

If we let

$$
\varphi_{1}\left(t^{\prime}\right) \equiv \inf _{t \geq t^{\prime}} \varphi(t)
$$


then it is easy to see that $\varphi_{1}(t) \leq \varphi(t)$ for all $t>0$ and hence $\|f\|_{\mathcal{M}_{q}^{\varphi_{1}}} \leq\|f\|_{\mathcal{M}_{q}^{\varphi}}$. Furthermore,

$$
\begin{aligned}
& \sup _{Q \in \mathcal{Q}} \varphi(\ell(Q))\left(\frac{1}{|Q|} \int_{Q}|f(x)|^{q} d x\right)^{\frac{1}{q}} \\
& \leq 2^{\frac{n}{q}} \sup _{Q \in \mathcal{Q}} \inf _{t^{\prime} \in(0, \ell(Q)]} \sup _{Q^{\prime} \in \mathcal{Q}: Q^{\prime} \subset Q, \ell\left(Q^{\prime}\right)=t^{\prime}} \varphi(\ell(Q))\left(\frac{1}{\left|Q^{\prime}\right|} \int_{Q^{\prime}}|f(x)|^{q} d x\right)^{\frac{1}{q}} \\
& \leq 2^{\frac{n}{q}} \sup _{Q \in \mathcal{Q}} \inf _{t^{\prime} \in(0, \ell(Q)]} \sup _{Q^{\prime} \in \mathcal{Q}: \ell\left(Q^{\prime}\right)=t^{\prime}} \varphi(\ell(Q))\left(\frac{1}{\left|Q^{\prime}\right|} \int_{Q^{\prime}}|f(x)|^{q} d x\right)^{\frac{1}{q}} \\
& =2^{\frac{n}{q}} \sup _{Q \in \mathcal{Q}} \inf _{t^{\prime} \in(0, \ell(Q)]} \sup _{Q^{\prime} \in \mathcal{Q}: \ell\left(Q^{\prime}\right)=t^{\prime}} \varphi_{1}\left(t^{\prime}\right)\left(\frac{1}{\left|Q^{\prime}\right|} \int_{Q^{\prime}}|f(x)|^{q} d x\right)^{\frac{1}{q}} \\
& =2^{\frac{n}{q}} \sup _{Q \in \mathcal{Q}} \inf _{t^{\prime} \in(0, \ell(Q)]} \sup _{Q^{\prime} \in \mathcal{Q}: \ell\left(Q^{\prime}\right)=t^{\prime}} \varphi_{1}\left(\ell\left(Q^{\prime}\right)\right)\left(\frac{1}{\left|Q^{\prime}\right|} \int_{Q^{\prime}}|f(x)|^{q} d x\right)^{\frac{1}{q}} \\
& \leq 2^{\frac{n}{q}}\|f\|_{\mathcal{M}_{q}^{\varphi_{1}} .}
\end{aligned}
$$

Thus it follows that

Next, if we let

$$
\|f\|_{\mathcal{M}_{q}^{\varphi_{1}}} \leq\|f\|_{\mathcal{M}_{q}^{\varphi}} \leq 2^{\frac{n}{q}}\|f\|_{\mathcal{M}_{q}^{\varphi_{1}}} .
$$

$$
\varphi^{*}(t) \equiv t^{\frac{n}{q}} \sup _{t^{\prime} \geq t} \varphi_{1}\left(t^{\prime}\right) t^{\prime-\frac{n}{q}}=\sup _{s \geq 1} \varphi_{1}(s t) s^{-\frac{n}{q}} \quad(t>0),
$$

then $\|f\|_{\mathcal{M}_{q}^{\varphi_{1}}}=\|f\|_{\mathcal{M}_{q}^{\varphi^{*}}}$. In fact, since $\varphi_{1}$ is increasing, $\varphi^{*}$ is increasing. From the definition of $\varphi^{*}, \varphi^{*}(t) \geq \varphi_{1}(t)$ for all $t>0$ and thus $\|f\|_{\mathcal{M}_{q}^{\varphi_{1}}} \leq\|f\|_{\mathcal{M}_{q}^{\varphi^{*}}}$. On the other hand, for any $r>0$ and $\varepsilon>0$, we can find $r^{\prime} \geq r$ such that

$$
\varphi^{*}(r) \leq(1+\varepsilon) r^{\frac{n}{q}} \varphi_{1}\left(r^{\prime}\right) r^{-\frac{n}{q}} .
$$

Thus for any cube $Q(x, r)$,

$$
\begin{aligned}
& \varphi^{*}(r)\left(\frac{1}{|Q(x, r)|} \int_{Q(x, r)}|f(y)|^{q} d y\right)^{\frac{1}{q}} \\
& \leq(1+\varepsilon) \varphi_{1}\left(r^{\prime}\right)\left(\frac{1}{\left|Q\left(x, r^{\prime}\right)\right|} \int_{Q(x, r)}|f(y)|^{q} d y\right)^{\frac{1}{q}} \\
& \leq(1+\varepsilon) \varphi_{1}\left(r^{\prime}\right)\left(\frac{1}{\left|Q\left(x, r^{\prime}\right)\right|} \int_{Q\left(x, r^{\prime}\right)}|f(y)|^{q} d y\right)^{\frac{1}{q}} \\
& \leq(1+\varepsilon)\|f\|_{\mathcal{M}_{q}^{\varphi_{1}}} .
\end{aligned}
$$

Taking the supremum over $x$ and $r$, we have $\|f\|_{\mathcal{M}_{q}^{\varphi^{*}}} \leq(1+\varepsilon)\|f\|_{\mathcal{M}_{q}^{\varphi_{1}}}$. Since $\varepsilon>0$ is arbitrary, we conclude $\|f\|_{\mathcal{M}_{q}^{\varphi^{*}}} \leq\|f\|_{\mathcal{M}_{q}^{\varphi_{1}}}$.

In view of Proposition 3.16, it follows that we can always suppose that $\varphi \in \mathcal{G}_{q}$. 
Remark 3.17. Some authors suppose that there exists $\delta>0$ such that

$$
\varphi(r) \leq \delta^{-1} \quad(0<r \leq 1)
$$

and that

$$
r^{\frac{n}{q}} \varphi(r)>\delta \quad(1<r<\infty) .
$$

As the next proposition shows, $\mathcal{G}_{q}$ is a good class in addition to the nice property that it naturally arises in generalized Morrey spaces.

Proposition 3.18. Let $\varphi \in \mathcal{G}_{q}$ with $0<q<\infty$. Then we can find a continuous function $\varphi^{*} \in \mathcal{G}_{q}$ such that $\varphi^{*}$ is strictly increasing and that $\varphi \sim \varphi^{*}$.

Proof. We look for $\varphi^{*}$ in a couple of steps.

- Consider

$$
\varphi_{0}(t) \equiv t^{\frac{n}{q}} \int_{t}^{2 t} \varphi(s) \frac{d s}{s^{\frac{n}{q}+1}}=\int_{1}^{2} \varphi(t s) \frac{d s}{s^{\frac{n}{q}+1}} \quad(t>0) .
$$

Since $\varphi$ is increasing, the function $\varphi_{0}$ is increasing. Also, by the fact that $\varphi$ is doubling, we see that $\varphi_{0}$ and $\varphi$ are equivalent. Thus, we may assume that $\varphi$ is continuous.

- We define

$$
\psi(t) \equiv \varphi(t) \chi_{(0,1]}(t)+\left(\frac{1-e^{-t}}{1-e^{-1}}\right)^{\frac{n}{q}} \varphi(1) \chi_{(1, \infty)}(t) \quad(t>0),
$$

Since we can check that $t \in(0,1] \mapsto t^{-\frac{n}{q}} \psi(t)$ and $t \in[1, \infty) \mapsto t^{-\frac{n}{q}} \psi(t)$ is both decreasing, $t \in(0, \infty) \mapsto t^{-\frac{n}{q}} \psi(t)$ is decreasing. Likewise we can check that $\psi$ is increasing. Thus, $\psi \in \mathcal{G}_{q}$. Since $\psi+\varphi \simeq \varphi$, we can assume that $\varphi$ is strictly increasing in $(1, \infty)$ and that $\varphi$ is continuous.

- Finally, we consider

$$
\varphi^{*}(t) \equiv \sum_{k=0}^{\infty} \frac{\varphi\left(2^{k} t\right)}{2^{k N}} \quad(t>0)
$$

where $N \equiv \frac{n}{q}+1$. Then it is easy to check that $\varphi^{*}$ is equivalent to $\varphi$ since each term is dominated by $2^{-k} \varphi$. Likewise since $\varphi \in \mathcal{G}_{q}, \varphi^{*} \in \mathcal{G}_{q}$. Finally since $\varphi$ is strictly increasing in $(1, \infty)$, the function $\varphi\left(2^{k} t\right)$ is strictly increasing on $\left(2^{-k}, \infty\right)$ for any $k \in \mathbb{N}$. Since $k \in \mathbb{N}$ is arbitrary, $\varphi^{*}$ is strictly increasing.

Thus, we are led to the following definition:

Definition 3.19. The class $W$ stands for the set of all continuous functions $\varphi$ : $(0, \infty) \rightarrow(0, \infty)$. That is, $W=C((0, \infty),(0, \infty))$.

We apply what we have obtained to small Morrey spaces. 
Example 3.20. Let $0<q<\infty$. Let us see how we modify $\psi$ in $\mathcal{M}_{q}^{\psi}\left(\mathbb{R}^{n}\right)$ to obtain the equivalent space $\mathcal{M}_{q}^{\varphi}\left(\mathbb{R}^{n}\right)$ with $\varphi \in \mathcal{G}_{q}$.

- Let $\varphi(t) \equiv \max \left(t^{\frac{n}{p}}, 1\right)$ and $\psi(t) \equiv t^{\frac{n}{p}} \chi_{(0,1]}(t)$ for $t>0$. Then with the equivalence of norms $m_{q}^{p}\left(\mathbb{R}^{n}\right)=\mathcal{M}_{q}^{\psi}\left(\mathbb{R}^{n}\right)=\mathcal{M}_{q}^{\varphi}\left(\mathbb{R}^{n}\right)$.

- Let $\varphi(t) \equiv \max \left(t^{a}, 1\right)$ with $a \geq \frac{n}{q}$ and $\psi(t) \equiv t^{\frac{n}{q}} \chi_{(0,1]}(t)$ for $t>0$. Then with the equivalence of norms $L_{\mathrm{uloc}}^{q}\left(\mathbb{R}^{n}\right)=\mathcal{M}_{q}^{\psi}\left(\mathbb{R}^{n}\right)=\mathcal{M}_{q}^{\varphi}\left(\mathbb{R}^{n}\right)$.

Note that $\varphi \in \mathcal{G}_{q} \cap W$ but that $\psi \in \mathcal{G}_{q} \backslash W$ in both cases.

So far we have shown that we may assume that $\varphi \in \mathcal{G}_{q}$. As a result, we may assume that $\varphi$ is doubling. This observation makes the definition of the norm $\|\cdot\|_{\mathcal{M}_{q}^{\varphi}}$ more flexible.

Remark 3.21. In (15), cubes can be replaced with balls; an equivalent norms will be obtained. Precisely use the norm given by

$$
\|f\|_{\mathcal{M}_{q}^{\varphi}} \equiv \sup _{x \in \mathbb{R}^{n}, r>0} \varphi(r)\left(\frac{1}{|B(x, r)|} \int_{B(x, r)}|f(y)|^{q} d y\right)^{\frac{1}{q}}
$$

to go through the same argument given for the norm defined by means of cubes.

Related to this definition, we give some definitions related to the class $\mathcal{G}_{q}$. Although we show that it is sufficient to limit ourselves to $\mathcal{G}_{q}$, we still feel that this class is too narrow as the function of $\varphi(t)=t \log \left(e+t^{-1}\right)$ shows. So, it is convenient to relax the condition on $\varphi$. The following definition will serve to this purpose.

Definition 3.22. Let $\ell>0$.

- A function $\varphi:(0, \ell] \rightarrow(0, \infty)$ is said to be almost decreasing if $\varphi(s) \lesssim \varphi(t)$ for all $0 \leq t<s \leq \ell$.

- A function $\varphi:(0, \ell] \rightarrow(0, \infty)$ is said to be almost increasing if $\varphi(s) \gtrsim \varphi(t)$ for all $0 \leq t<s \leq \ell$.

- A function $\varphi:(0, \infty) \rightarrow(0, \infty)$ is said to be almost decreasing if $\varphi(s) \lesssim \varphi(t)$ for all $0 \leq t<s<\infty$.

- A function $\varphi:(0, \infty) \rightarrow(0, \infty)$ is said to be almost increasing if $\varphi(s) \gtrsim \varphi(t)$ for all $0 \leq t<s<\infty$.

The implicit constants in these inequality are called the almost decreasing/increasing constants.

\section{Example 3.23.}

- Let $a, b$ be real parameters with $b \neq 0$. Let $\varphi_{a, b}(t)=t^{a}(\log (e+t))^{b}$ for $t>0$. Then $\varphi_{a, b}$ is almost increasing for any $a>0$. If $a<0$, then $\varphi_{a, b}$ is almost decreasing.

- The function $\varphi(t)=t+\sin 2 t, t>0$ is almost increasing but not increasing.

- The function $\varphi(t)=1+e^{t}(1-\cos t), t>0$ is not almost increasing because $\varphi(2 \pi m)=1$ and $\varphi(2 \pi m+\pi)=1+2 e^{2 \pi m+\pi}$ for all $m \in \mathbb{N}$. 
- Let $0<p, q<\infty$ and $\beta_{1}, \beta_{2} \in \mathbb{R}$. We write

$$
\ell^{\mathbb{B}}(r) \equiv \begin{cases}(1+|\log r|)^{\beta_{1}} & (0<r \leq 1), \\ (1+|\log r|)^{\beta_{2}} & (1<r<\infty) .\end{cases}
$$

We set

$$
\varphi(t)=t^{\frac{n}{p}} \ell^{-\mathbb{B}}(t) \quad(t>0)
$$

as we did in Example 3.10. We observe that $\varphi$ is almost increasing for all $p>0$ and $\beta_{1}, \beta_{2} \in \mathbb{R}$. We also note that $t \mapsto \varphi(t) t^{-\frac{n}{q}}$ is almost decreasing if and only if $p=q$ and $\beta_{1} \leq 0 \leq \beta_{2}$ or $p>q$. Note that $\varphi$ is an equivalent to a function $\psi \in \mathcal{G}_{q}$ if and only if either $p=q, \beta_{1} \leq 0 \leq \beta_{2}$ or $p>q$ since we can neglect the effect of "log".

- Let $E \subset(0, \infty)$ be a non-Lebegsgue measurable set. Then $\varphi=1+\chi_{E}$ is almost increasing and almost decreasing. Note that $\varphi$ is not measurable.

- A simple but still standard example is as follows: Let $m \in \mathbb{N}$ and define

$$
\varphi(t) \equiv \frac{t^{\frac{n}{q}}}{l_{m}(t)} \quad(t>0)
$$

where $l_{m}(t)$ is given inductively by:

$$
l_{0}(t) \equiv t, \quad l_{m}(t) \equiv \log \left(3+l_{m-1}(t)\right) \quad(m=1,2, \ldots)
$$

for $t>0$. The for all $0<q<\infty$ and $m \in \mathbb{N}, \varphi \in \mathcal{G}_{q}$.

As the following lemma shows, we can always replace an almost increasing function with an increasing function.

Lemma 3.24. If a function $\varphi:(0, \infty) \rightarrow(0, \infty)$ is almost increasing with the almost increasing constant $C_{0}>0$, then there exists an increasing function $\psi$ : $(0, \infty) \rightarrow(0, \infty)$ such that $\varphi(t) \leq \psi(t) \leq C_{0} \varphi(t)$ for all $t>0$.

Proof. Simply set $\psi(t)=\sup _{0<s \leq t} \varphi(s), t>0$.

Having set down the condition of $\varphi$, we move on to the norm estimate of the function. The lemma below gives an estimate for the norm of $\chi_{B(R)}$ in $\mathcal{M}_{q}^{\varphi}\left(\mathbb{R}^{n}\right)$.

Lemma 3.25. [60, Lemma 4.1] Let $0<q<\infty$ and $\varphi \in \mathcal{G}_{q}$. Then $\left\|\chi_{Q(x, R)}\right\|_{\mathcal{M}_{q}^{\varphi}}=$ $\varphi(R)$ for all $R>0$.

Proof. One method is to reexamine Example 3.6. Here we give a direct proof. It is easy to see that $\left\|\chi_{Q(x, R)}\right\|_{\mathcal{M}_{q}^{\varphi}} \geq \varphi(R)$. To prove the opposite inequality we consider

$$
\varphi(r) \frac{1}{|Q(y, r)|^{\frac{1}{q}}}\left\|\chi_{Q(y, r) \cap Q(x, R)}\right\|_{q}
$$

for any cube $Q=Q(y, r)$. When $R \leq r$, then

$$
\varphi(r) \frac{\left\|\chi_{Q(y, r) \cap Q(x, R)}\right\|_{q}}{|Q(y, r)|^{\frac{1}{q}}} \leq \varphi(r) \frac{\left\|\chi_{Q(x, R)}\right\|_{q}}{|Q(y, r)|^{\frac{1}{q}}} \leq \varphi(R) \frac{\left\|\chi_{Q(x, R)}\right\|_{q}}{|Q(y, R)|^{\frac{1}{q}}}=\varphi(R) .
$$


since $\varphi \in \mathcal{G}_{q}$. When $R>r$, then

$$
\varphi(r) \frac{\left\|\chi_{Q(y, r) \cap Q(x, R)}\right\|_{q}}{|Q(y, r)|^{\frac{1}{q}}} \leq \varphi(r) \frac{\left\|\chi_{Q(y, r)}\right\|_{q}}{|Q(y, r)|^{\frac{1}{q}}}=\varphi(r) \leq \varphi(R)
$$

again by virtue of the fact that $\varphi \in \mathcal{G}_{q}$.

A direct consequence of the above quantitative information is:

Corollary 3.26. [80, Corollary 2.3] Let $0<q<\infty$ and $\varphi:(0, \infty) \rightarrow(0, \infty)$ be a function in the class $\mathcal{G}_{q}$. If $N_{0}>n / q$, then $(1+|\cdot|)^{-N_{0}} \in \mathcal{M}_{q}^{\varphi}\left(\mathbb{R}^{n}\right)$.

Proof. Since $\varphi \in \mathcal{G}_{q}$, we have $\varphi(t) t^{-\frac{n}{q}} \leq \varphi(1)$ for all $t \geq 1$. we have

$$
\left\|(1+|\cdot|)^{-N_{0}}\right\|_{\mathcal{M}_{q}^{\varphi}} \lesssim \sum_{j=1}^{\infty} \frac{\left\|\chi_{Q(j)}\right\|_{\mathcal{M}_{q}^{\varphi}}}{\max (1, j-1)^{N_{0}}} \leq \sum_{j=1}^{\infty} \frac{\varphi(j)}{\max (1, j-1)^{N_{0}}}<\infty .
$$

Here for the second inequality we invoked Lemma 3.25.

Now we consider the role of $\varphi$. We did not tolerate the case where $p=\infty$ when we define $\mathcal{M}_{q}^{p}\left(\mathbb{R}^{n}\right)$. If we define $\mathcal{M}_{q}^{\infty}\left(\mathbb{R}^{n}\right)$ similar to $\mathcal{M}_{q}^{p}\left(\mathbb{R}^{n}\right)$, then we have $\mathcal{M}_{q}^{\infty}\left(\mathbb{R}^{n}\right)=L^{\infty}\left(\mathbb{R}^{n}\right)$ by the Lebesgue differentiation theorem. The next theorem concerns a situation close to this.

Theorem 3.27. [75, Proposition 3.3] Let $0<q<\infty$ and $\varphi \in \mathcal{G}_{q}$. Then, the following are equivalent:

- $\inf _{t>0} \varphi(t)>0$

- $\mathcal{M}_{q}^{\varphi}\left(\mathbb{R}^{n}\right) \subset L^{\infty}\left(\mathbb{R}^{n}\right)$.

If these conditions are satisfied, then $\mathcal{M}_{q}^{\varphi}\left(\mathbb{R}^{n}\right)=L^{\infty}\left(\mathbb{R}^{n}\right) \cap \mathcal{M}_{q}^{\varphi-\inf _{t>0} \varphi(t)}\left(\mathbb{R}^{n}\right)$ with equivalence of norms.

Proof. If $\inf _{t>0} \varphi(t)>0$, then by the Lebesgue differentiation theorem we get

$$
\begin{aligned}
|f(x)|^{q} & =\lim _{r \downarrow 0} \frac{1}{|B(x, r)|} \int_{B(x, r)}|f(y)|^{q} d y \\
& \leq \frac{1}{\inf _{t>0} \varphi(t)^{q}} \lim _{r \downarrow 0} \frac{\varphi(r)^{q}}{|B(x, r)|} \int_{B(x, r)}|f(y)| d y \\
& \leq \frac{1}{\inf _{t>0} \varphi(t)^{q}}\|f\|_{\mathcal{M}_{q}^{\varphi}}{ }^{q}
\end{aligned}
$$

for all $f \in \mathcal{M}_{q}^{\varphi}\left(\mathbb{R}^{n}\right)$ and all Lebesgue points $x$ of $|f|^{q}$. Therefore, $f \in L^{\infty}$.

Assume that $\mathcal{M}_{q}^{\varphi}\left(\mathbb{R}^{n}\right) \subset L^{\infty}\left(\mathbb{R}^{n}\right)$. Then by the closed graph theorem, $\|f\|_{\infty} \lesssim\|f\|_{\mathcal{M}_{q}^{\varphi}}$ for all $f \in \mathcal{M}_{q}^{\varphi}\left(\mathbb{R}^{n}\right)$. If we choose $f=\chi_{B(a, r)}$, then we have $1 \lesssim \varphi(r)$. This shows that $\inf _{t>0} \varphi(t)>0$. 
Finally, by taking $\varphi_{1} \equiv \inf _{t>0} \varphi(t)$ and $\varphi_{2} \equiv \varphi-\varphi_{1}$, we obtain $\mathcal{M}_{q}^{\varphi}\left(\mathbb{R}^{n}\right)=$ $L^{\infty}\left(\mathbb{R}^{n}\right) \cap \mathcal{M}_{q}^{\varphi-\inf _{t>0} \varphi(t)}\left(\mathbb{R}^{n}\right)$ with equivalence of norms from the general formula $\mathcal{M}_{q}^{\varphi_{1}+\varphi_{2}}\left(\mathbb{R}^{n}\right)=\mathcal{M}_{q}^{\varphi_{1}}\left(\mathbb{R}^{n}\right) \cap \mathcal{M}_{q}^{\varphi_{2}}\left(\mathbb{R}^{n}\right)$ with equivalence of norms.

We also investigate the reverse inclusion to Theorem 3.27.

Theorem 3.28. [75, Proposition 3.3] Let $0<q<\infty$ and $\varphi \in \mathcal{G}_{q}$. Then, the following are equivalent:

- $\sup \varphi(t)<\infty$

- $\mathcal{M}_{q}^{\varphi}\left(\mathbb{R}^{n}\right) \supset L^{\infty}\left(\mathbb{R}^{n}\right)$.

Proof. If $\sup _{t>0} \varphi(t)<\infty$, then by letting $\psi(t) \equiv \sup _{s>0} \varphi(s), t>0$ we have $L^{\infty}\left(\mathbb{R}^{n}\right)=$ $\mathcal{M}_{q}^{\psi}\left(\mathbb{R}^{n}\right)$ with equivalence of norms. Since $\varphi \leq \psi$, we have $\mathcal{M}_{q}^{\psi}\left(\mathbb{R}^{n}\right) \subset \mathcal{M}_{q}^{\varphi}\left(\mathbb{R}^{n}\right)$. Thus $L^{\infty}\left(\mathbb{R}^{n}\right) \subset \mathcal{M}_{q}^{\varphi}\left(\mathbb{R}^{n}\right)$.

Conversely, if $L^{\infty}\left(\mathbb{R}^{n}\right) \subset \mathcal{M}_{q}^{\varphi}\left(\mathbb{R}^{n}\right)$, then by the closed graph theorem the embedding norm is finite and hence $\varphi(r)=\left\|\chi_{Q(r)}\right\|_{\mathcal{M}_{q}^{\varphi}} \lesssim\left\|\chi_{Q(r)}\right\|_{L^{\infty}}=1$ for all $r>0$. Then $\sup \varphi<\infty$.

By combining these two theorems, we obtain the following result.

Corollary 3.29. [75, Proposition 3.3] Let $0<q<\infty$ and $\varphi \in \mathcal{G}_{q}$. Then, the following are equivalent:

- $\log \varphi \in L^{\infty}(0, \infty)$, i.e., $0<\inf _{t>0} \varphi(t) \leq \sup _{t>0} \varphi(t)<\infty$,

- $L^{\infty}\left(\mathbb{R}^{n}\right)=\mathcal{M}_{q}^{\varphi}\left(\mathbb{R}^{n}\right)$. $L^{\infty}\left(\mathbb{R}^{n}\right)$.

So, if $\log \varphi$ grows or decays slowly we can say that $\mathcal{M}_{q}^{\varphi}\left(\mathbb{R}^{n}\right)$ is close to

The next example shows that when the support of the functions are torn apart, the norm does not increase even in the case of generalized Morrey spaces.

Example 3.30. Let $0<q<\infty$ and $\varphi \in \mathcal{G}_{q}$. Suppose that we have a collection of cubes $\left\{Q_{j}\right\}_{j=1}^{\infty}=\left\{Q\left(a_{j}, r_{j}\right)\right\}_{j=1}^{\infty}$ such that $\left\{3 Q_{j}\right\}_{j=1}^{\infty}=\left\{Q\left(a_{j}, 3 r_{j}\right)\right\}_{j=1}^{\infty}$ is disjoint and is contained in $Q=Q\left(a_{0}, r_{0}\right)$. Let $\left\{f_{j}\right\}_{j=1}^{\infty}$ be a collection of functions in $\mathcal{M}_{q}^{\varphi}\left(\mathbb{R}^{n}\right)$ satisfying

$$
\begin{gathered}
\left\|f_{j}\right\|_{q} \leq \varphi\left(r_{0}\right)^{-1} r_{j}{ }^{\frac{n}{q}}, \quad \operatorname{supp}\left(f_{j}\right) \subset Q_{j}, \\
\left\|f_{j}\right\|_{\mathcal{M}_{q}^{\varphi}} \leq 1
\end{gathered}
$$

for each $j \in \mathbb{N}$. Then $f \equiv \sum_{j=1}^{\infty} f_{j} \in \mathcal{M}_{q}^{\varphi}\left(\mathbb{R}^{n}\right)$. Since $f$ is supported in $Q$, we have only to consider cubes contained in $3 Q$; if a cube $Q^{\prime}$ intersects both $Q$ and $\mathbb{R}^{n} \backslash 3 Q$, then its triple $3 Q^{\prime}$ engulfs $Q$ and the radius of $Q$ is smaller than that of $Q^{\prime}$. Thus 
we have

$$
\|f\|_{\mathcal{M}_{q}^{\varphi}} \lesssim \sup _{x \in \mathbb{R}^{n}, r>0, Q(x, r) \subset 3 Q} \varphi(r)\left(\frac{1}{|Q(x, r)|} \int_{Q(x, r)}|f(y)|^{q} d y\right)^{\frac{1}{q}} .
$$

We fix a cube $Q(x, r)$ and estimate

$$
\varphi(r)\left(\frac{1}{|Q(x, r)|} \int_{Q(x, r)}|f(y)|^{q} d y\right)^{\frac{1}{q}} .
$$

We let

$$
\begin{aligned}
& J_{1} \equiv\left\{j \in \mathbb{N}: Q(x, r) \cap Q\left(a_{j}, r_{j}\right) \neq \emptyset, r_{j} \leq r\right\}, \\
& J_{2} \equiv\left\{j \in \mathbb{N}: Q(x, r) \cap Q\left(a_{j}, r_{j}\right) \neq \emptyset, r_{j}>r\right\} .
\end{aligned}
$$

Accordingly we set

$$
\mathrm{I}_{i} \equiv \varphi(r)\left(\frac{1}{|Q(x, r)|} \int_{Q(x, r)}\left|\sum_{j \in J_{i}} f_{j}(y)\right|^{q} d y\right)^{\frac{1}{q}}
$$

for $i=1,2$.

As for $I_{1}$, we use (23) and the fact that $\left\{Q\left(a_{j}, r_{j}\right)\right\}_{j=1}^{\infty}$ is disjoint:

$$
\mathrm{I}_{1} \leq \varphi(r)\left(\frac{1}{|Q(x, r)|} \sum_{j \in J_{1}} \varphi\left(r_{0}\right)^{-q} r_{j}{ }^{n}\right)^{\frac{1}{q}} \leq\left(\frac{1}{|Q(x, r)|} \sum_{j \in J_{1}} r_{j}{ }^{n}\right)^{\frac{1}{q}} \lesssim 1 .
$$

As for $I_{2}$, we have only to consider only one summand, since $Q\left(a_{j}, 3 r_{j}\right)$ engulfs $Q(x, r)$ if $j \in H_{2}$ : we can deduce $\mathrm{I}_{2} \leq 1$ using (24).

As an application of Example 3.30, we present another example. Denote by $[t]$ the integer part of $t \in \mathbb{R}$. For positive sequences $\left\{A_{k}\right\}_{k=1}^{\infty}$ and $\left\{B_{k}\right\}_{k=1}^{\infty} " A_{k} \sim B_{k}$ as $k \rightarrow \infty$ "means that $\left\{\log \left(A_{k} / B_{k}\right)\right\}_{k=1}^{\infty}$ is a bounded sequence.

Example 3.31. [75, Lemma 4.1] Let $\varphi \in \mathcal{G}_{q}$ with $0<q<\infty$. We fix $a \in \mathbb{R}^{n}$. Let $\left\{s_{k}\right\}_{k=1}^{\infty} \subset(0,1]$ be a sequence which decreases to 0 .

Keeping in mind

$$
\inf _{0<t \leq 1} \varphi(t)^{q / n} t^{-1}=\varphi(1)^{q / n}, \quad \inf _{0<t<1} \varphi(t)^{-q / n}=\varphi(1)^{-q / n},
$$

we let

$$
\begin{aligned}
\ell_{k} & \equiv\left[1+\varphi\left(s_{k}\right)^{q / n} s_{k}{ }^{-1}\right]\left(\sim \varphi\left(s_{k}\right)^{q / n} s_{k}{ }^{-1}\right) \\
m_{k} & \equiv\left[1+\varphi\left(s_{k}\right)^{-q / n}\right]\left(\sim \varphi\left(s_{k}\right)^{-q / n}\right) .
\end{aligned}
$$

Then

$$
\ell_{k} s_{k} m_{k} \sim \varphi\left(s_{k}\right)^{\frac{q}{n}} s_{k}{ }^{-1} s_{k} \varphi\left(s_{k}\right)^{-\frac{q}{n}}=1, \quad s_{k} \downarrow 0
$$

as $k \rightarrow \infty$. Hence

$$
\varphi\left(\left(\ell_{k} m_{k}\right)^{-1}\right)^{-q} m_{k}{ }^{-n} \sim \varphi\left(s_{k}\right)^{-q} s_{k}{ }^{n} \ell_{k}{ }^{n} \sim 1
$$


as $k \rightarrow \infty$.

Devide equally $a+[0,1]^{n}$ into $\ell_{k}^{n}$ cubes to have a non-overlapping collection

$$
\left\{b_{k, j}+\left[0, \ell_{k}{ }^{-1}\right]^{n}\right\}_{j=1,2, \ldots, \ell_{k}{ }^{n}} .
$$

Furthermore, we devide $\left[0, \ell_{k}{ }^{-1}\right]^{n}$ equally into $m_{k}{ }^{n}$ cubes to obtain a collection

$$
\left\{e_{k, j}+\left[0, m_{k}{ }^{-1}\right]^{n}\right\}_{j=1,2, \ldots, m_{k}{ }^{n}}
$$

of cubes. Then

$$
b_{k, j}+\left[0, \ell_{k}^{-1}\right]^{n}=\bigcup_{i=1}^{m_{k}{ }^{n}}\left(b_{k, j}+e_{k, i}+\left[0,\left(\ell_{k} m_{k}\right)^{-1}\right]^{n}\right) .
$$

If we set

$$
g_{k, i, j, a}=\frac{1}{\varphi\left(\left(\ell_{k} m_{k}\right)^{-1}\right)} \chi_{b_{k, j}+e_{k, i}+\left[0,\left(\ell_{k} m_{k}\right)^{-1}\right]^{n}} .
$$

and

$$
f_{k, i, a}=f_{k, i}=\sum_{j=1}^{\ell_{k}{ }^{n}} \frac{\chi_{b_{k, j}+e_{k, i}+\left[0,\left(\ell_{k} m_{k}\right)^{-1}\right]^{n}}}{\varphi\left(\left(\ell_{k} m_{k}\right)^{-1}\right)}=\sum_{j=1}^{\ell_{k}{ }^{n}} g_{k, i, j, a} .
$$

Then, each $f_{k, i, a}$ is supported in $a+[0,1]^{n}$ and each $g_{k, i, j, a}$ is supported in $b_{k, j}+$

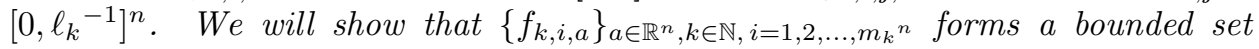
in $\mathcal{M}_{q}^{\varphi}\left(\mathbb{R}^{n}\right)$. This is achieved by verifying the assumption of Example 3.30. Let $F_{1}, F_{2}, \ldots, F_{3^{n}}$ be a partition of $\left\{1,2, \ldots, \ell_{k}{ }^{n}\right\}$ such that

$$
\left\{b_{k, j}+\left[-\ell_{k}^{-1}, 2 \ell_{k}^{-1}\right]^{n}\right\}_{j \in F_{l^{\prime}}{ }^{n}}
$$

is not overlapping for each $l^{\prime}=1,2, \ldots, 3^{n}$. To check this we need to verify (23) and (24).

From Lemma 3.25, we have (23). Meanwhile,

$$
\left\|g_{k, i, j, a}\right\|_{q}=\varphi\left(\left(\ell_{k} m_{k}\right)^{-1}\right)\left(\ell_{k} m_{k}\right)^{-\frac{n}{q}} \sim \ell_{k}^{-\frac{n}{q}} .
$$

Thus for each $l^{\prime}=1,2, \ldots, 3^{n}$

$$
\left\{\frac{1}{\varphi\left(\left(\ell_{k} m_{k}\right)^{-1}\right)} \sum_{j \in F_{l^{\prime}}} \chi_{b_{k, j}+e_{k, i}+\left[0,\left(\ell_{k} m_{k}\right)^{-1}\right]^{n}}\right\}_{a \in \mathbb{R}^{n}, k \in \mathbb{N}, i=1,2, \ldots, m_{k}{ }^{n}}
$$

is a bounded set. So $\left\{f_{k, i, a}\right\}_{a \in \mathbb{R}^{n}, k \in \mathbb{N}, i=1,2, \ldots, m_{k}{ }^{n}}$ forms a bounded set in $\mathcal{M}_{q}^{\varphi}\left(\mathbb{R}^{n}\right)$.

From these examples, we obtain the following conclusion:

Corollary 3.32. [75, Corollary 4.11] Let $0<q_{1}, q_{2}<\infty$, and let $\varphi_{1} \in \mathcal{G}_{q_{1}}$ and $\varphi_{2} \in \mathcal{G}_{q_{2}}$. Assume in addition that $\inf _{t>0} \varphi_{1}(t)=0$. Then $\mathcal{M}_{q_{1}}^{\varphi_{1}}\left(\mathbb{R}^{n}\right) \subset \mathcal{M}_{q_{2}}^{\varphi_{2}}\left(\mathbb{R}^{n}\right)$ if and only if $q_{1} \geq q_{2}$ and $\varphi_{1} \gtrsim \varphi_{2}$. Furthemore, if $q_{1}<q_{2}$, there exists a compactly supported function $f \in \mathcal{M}_{q_{2}}^{\varphi_{2}}\left(\mathbb{R}^{n}\right) \backslash \mathcal{M}_{q_{1}}^{\varphi_{1}}\left(\mathbb{R}^{n}\right)$. In particular, for $0<q_{1} \leq p_{1}<\infty$ and $0<q_{2} \leq p_{2}<\infty, \mathcal{M}_{q_{1}}^{p_{2}}\left(\mathbb{R}^{n}\right) \subset \mathcal{M}_{q_{2}}^{p_{2}}\left(\mathbb{R}^{n}\right)$ if and only if $q_{1} \geq q_{2}$ and $p_{1}=p_{2}$. 
Proof. The "if" part is trivial thanks to Lemma 3.12. Let us concentrate on the "only if" part.

Assume $q_{1}<q_{2}$. We employ Example 3.31 with $q=q_{1}$ and $\varphi=\varphi_{1}$. Then

$$
\left\|\sum_{j \in F_{l^{\prime}}} \chi_{b_{k, j}+e_{k, i}+\left[0,\left(\ell_{k} m_{k}\right)^{-1}\right]^{n}}\right\|_{L^{q_{2}}} \sim\left(\ell_{k}^{n}\left(\ell_{k} m_{k}\right)^{-n}\right)^{\frac{1}{q_{2}}}=m_{k}^{-\frac{n}{q_{2}}} .
$$

As a result,

$$
\begin{aligned}
\frac{1}{\varphi\left(\left(\ell_{k} m_{k}\right)^{-1}\right)}\left\|\sum_{j \in F_{l^{\prime}}} \chi_{b_{k, j}+e_{k, i}+\left[0,\left(\ell_{k} m_{k}\right)^{-1}\right]^{n}}\right\|_{L^{q_{2}}} \sim \frac{1}{\varphi\left(\left(\ell_{k} m_{k}\right)^{-1}\right) m_{k}^{\frac{n}{q_{2}}}} \\
=m_{k}^{\frac{n}{q}-\frac{n}{q_{2}}} \rightarrow \infty,
\end{aligned}
$$

since $\inf _{t>0} \varphi_{1}(t)=0$. Thus, $\mathcal{M}_{q_{1}}^{\varphi_{1}}\left(\mathbb{R}^{n}\right)$ contains a function which does not belong to $L_{\text {loc }}^{q_{2}}\left(\mathbb{R}^{n}\right)$. Consequently, if $\mathcal{M}_{q_{1}}^{\varphi_{1}}\left(\mathbb{R}^{n}\right) \subset \mathcal{M}_{q_{2}}^{\varphi_{2}}\left(\mathbb{R}^{n}\right)$, then $q_{1} \geq q_{2}$. Finally, we must have $\varphi_{1} \gtrsim \varphi_{2}$ from Lemma 3.25 if $\mathcal{M}_{q_{1}}^{\varphi_{1}}\left(\mathbb{R}^{n}\right) \subset \mathcal{M}_{q_{2}}^{\varphi_{2}}\left(\mathbb{R}^{n}\right)$.

When $\inf _{t>0} \varphi_{1}(t)>0$, the situation is different.

Example 3.33. When $\varphi_{1}$ is constant, we use Theorem 3.28 to have the following characterization. Let $0<q_{1}, q_{2}<\infty$, and let $\varphi_{1}=1 \in \mathcal{G}_{q_{1}}$ and $\varphi_{2} \in \mathcal{G}_{q_{2}}$. Then $L^{\infty}\left(\mathbb{R}^{n}\right)=\mathcal{M}_{q_{1}}^{\varphi_{1}}\left(\mathbb{R}^{n}\right) \subset \mathcal{M}_{q_{2}}^{\varphi_{2}}\left(\mathbb{R}^{n}\right)$ if and only if $\sup _{t>0} \varphi_{2}(t)<\infty$.

For the later purpose we use the following characterization of $\mathcal{M}_{q}^{\varphi}\left(\mathbb{R}^{n}\right)$, which is defined to be the closure of $\mathcal{M}_{q}^{\varphi}\left(\mathbb{R}^{n}\right) \cap L_{\mathrm{c}}^{0}\left(\mathbb{R}^{n}\right)$ in $\mathcal{M}_{q}^{\varphi}\left(\mathbb{R}^{n}\right)$.

Lemma 3.34. For $0<q \leq p<\infty$, we have

$$
\mathcal{M}_{q}^{\varphi}\left(\mathbb{R}^{n}\right)=\left\{f \in \mathcal{M}_{q}^{\varphi}\left(\mathbb{R}^{n}\right): \lim _{R \rightarrow \infty}\left\|\chi_{\mathbb{R}^{n} \backslash B(R)} f\right\|_{\mathcal{M}_{q}^{\varphi}}=0\right\} .
$$

Proof. Assume that $f \in \mathcal{M}_{q}^{\varphi}\left(\mathbb{R}^{n}\right)$ satisfies

$$
\lim _{R \rightarrow \infty}\left\|\chi_{\mathbb{R}^{n} \backslash B(R)} f\right\|_{\mathcal{M}_{q}^{\varphi}}=0 .
$$

Then $f=\lim _{R \rightarrow \infty} \chi_{B(R)} f$ in $\mathcal{M}_{q}^{\varphi}\left(\mathbb{R}^{n}\right)$. Conversely, if $f \in \mathcal{M}_{q}^{\varphi}\left(\mathbb{R}^{n}\right)$ and $\varepsilon>0$, then we can find $g \in \mathcal{M}_{q}^{\varphi}\left(\mathbb{R}^{n}\right) \cap L_{\mathrm{c}}^{0}\left(\mathbb{R}^{n}\right)$ such that $\|f-g\|_{\mathcal{M}_{q}^{\varphi}}<\varepsilon$. By the triangle inequality

$$
\left\|\chi_{\mathbb{R}^{n} \backslash B(R)} f\right\|_{\mathcal{M}_{q}^{\varphi}} \lesssim\left\|\chi_{\mathbb{R}^{n} \backslash B(R)} g\right\|_{\mathcal{M}_{q}^{\varphi}}+\|f-g\|_{\mathcal{M}_{q}^{\varphi}} .
$$

Let $R>0$ be large enough as to have $\operatorname{supp}(g) \subset B(R)$. Then the above inequality reads as:

as required.

$$
\left\|\chi_{\mathbb{R}^{n} \backslash B(R)} f\right\|_{\mathcal{M}_{q}^{\varphi}} \lesssim\|f-g\|_{\mathcal{M}_{q}^{\varphi}}(<\varepsilon),
$$

We also have the following characterization of the space $\overline{\mathcal{M}}_{q}^{\varphi}\left(\mathbb{R}^{n}\right)$, the closure of $L^{\infty}\left(\mathbb{R}^{n}\right) \cap \mathcal{M}_{q}^{\varphi}\left(\mathbb{R}^{n}\right)$ in $\mathcal{M}_{q}^{\varphi}\left(\mathbb{R}^{n}\right)$ : 
Lemma 3.35. Let $0<q<\infty$ and $\varphi \in \mathcal{G}_{q}$. If $f \in \overline{\mathcal{M}}_{q}^{\varphi}\left(\mathbb{R}^{n}\right)$, then

$$
\lim _{R \rightarrow \infty}\left\|\chi_{\{|f|>R\}} f\right\|_{\mathcal{M}_{q}^{\varphi}}=0 .
$$

Proof. Let $\varepsilon>0$ be fixed. Since $f \in \overline{\mathcal{M}}_{q}^{\varphi}\left(\mathbb{R}^{n}\right)$, we can choose $g \in L^{\infty}\left(\mathbb{R}^{n}\right) \cap$ $\mathcal{M}_{q}^{\varphi}\left(\mathbb{R}^{n}\right)$ so that $\|g-f\|_{\mathcal{M}_{q}^{\varphi}}<\varepsilon$. Let $R>\|g\|_{\infty}$ We estimate

$$
\left\|\chi_{\{|f|>2 R\}} f\right\|_{\mathcal{M}_{q}^{\varphi}} \lesssim\|f-g\|_{\mathcal{M}_{q}^{\varphi}}+\left\|\chi_{\{|f|>2 R\}} g\right\|_{\mathcal{M}_{q}^{\varphi}}
$$

If $|g| \leq R$ and $|f|>2 R$, then $|g| \leq R \leq|f-g|$. Thus

$$
\left\|\chi_{\{|f|>2 R\}} f\right\|_{\mathcal{M}_{q}^{\varphi}} \lesssim 2\|f-g\|_{\mathcal{M}_{q}^{\varphi}}+\left\|\chi_{\{|g|>R\}} g\right\|_{\mathcal{M}_{q}^{\varphi}} \leq 2 \varepsilon .
$$

As a result (29) holds.

The generalized tilde subspace $\widetilde{\mathcal{M}}_{q}^{\varphi}\left(\mathbb{R}^{n}\right)$ is defined to be the completion of $L^{\infty}\left(\mathbb{R}^{n}\right) \cap \mathcal{M}_{q}^{\varphi}\left(\mathbb{R}^{n}\right)$ in $\mathcal{M}_{q}^{\varphi}\left(\mathbb{R}^{n}\right)$.

Proposition 3.36. Let $0<q<\infty$ and $\varphi \in \mathcal{G}_{q}$. Then $\widetilde{\mathcal{M}}_{q}^{\varphi}\left(\mathbb{R}^{n}\right)=\overline{\mathcal{M}}_{q}^{\varphi}\left(\mathbb{R}^{n}\right) \cap$ $\stackrel{\mathcal{M}}{q}_{q}^{\varphi}\left(\mathbb{R}^{n}\right)$.

Proof. It is easy to show that $\widetilde{\mathcal{M}}_{q}^{\varphi}\left(\mathbb{R}^{n}\right) \subset \overline{\mathcal{M}}_{q}^{\varphi}\left(\mathbb{R}^{n}\right) \cap \mathcal{M}_{q}^{\varphi}\left(\mathbb{R}^{n}\right)$. Let $f \in \overline{\mathcal{M}}_{q}^{\varphi}\left(\mathbb{R}^{n}\right) \cap$ $\mathcal{M}_{q}^{*}\left(\mathbb{R}^{n}\right)$. By Lemmas 3.34 and 3.35 , we see that

$$
f=\lim _{R \rightarrow \infty} \chi_{B(R) \cap\{|f|>R\}} f .
$$

Thus $f \in \widetilde{\mathcal{M}}_{q}^{\varphi}\left(\mathbb{R}^{n}\right)$.

Although we can define many other closed subspaces as we did for Morrey spaces, we content ourselves with these three definitions, which we actually use.

Remark 3.37. Here the space $\widetilde{\mathcal{M}}_{q}^{\varphi}\left(\mathbb{R}^{n}\right)$ is introduced. See $[3,12,66,88,89]$ for a different class of closed subspaces.

\section{Boundedness properties of the operators in Generalized Morrey SPACES}

Having set down the boundedness properties of generalized Morrey spaces, we are now interested in the boundedness properties of the operators.

4.1. Maximal operator in generalized Morrey spaces and the class $\mathbb{Z}_{0}$. After we generalize the parameter $p$ in the space $\mathcal{M}_{q}^{p}\left(\mathbb{R}^{n}\right)$, we realize that the boundedness of the maximal operator is obtained due to the condition on $q \in(1, \infty)$.

Theorem 4.1. [73, Theorem 1], [75, Corollary 5.6], [91, Theorem 2.3] Let $1<q<$ $\infty$ and $\varphi \in \mathcal{G}_{q}$. Then

$$
\|M f\|_{\mathcal{M}_{q}^{\varphi}} \lesssim\|f\|_{\mathcal{M}_{q}^{\varphi}}
$$

for all measurable functions $f$. 
We observe that we did not requere anything other than $\varphi \in \mathcal{G}_{q}$ as an evidence of the fact that the parametre $q$ play a central role for the boundedness of the Hardy-Littlewood maximal operator. Theorem 4.1 extends the result in [10] from Morrey spaces to generalized Morrey spaces.

Proof. Once we assume $\varphi \in \mathcal{G}_{q}$, the proof of this theorem will be an adaptation of the classical case. Fix a cube $Q(x, r)$. We need to prove

$$
\varphi(r)\left(\frac{1}{|Q(x, r)|} \int_{Q(x, r)} M f(y)^{q} d y\right)^{\frac{1}{q}} \lesssim\|f\|_{\mathcal{M}_{q}^{\varphi}} .
$$

We let $f_{1} \equiv \chi_{Q(x, 5 r)} f$ and $f_{2} \equiv f-f_{1}$. We need to prove

$$
\varphi(r)\left(\frac{1}{|Q(x, r)|} \int_{Q(x, r)} M f_{1}(y)^{q} d y\right)^{\frac{1}{q}} \lesssim\|f\|_{\mathcal{M}_{q}^{\varphi}}
$$

and

$$
\varphi(r)\left(\frac{1}{|Q(x, r)|} \int_{Q(x, r)} M f_{2}(y)^{q} d y\right)^{\frac{1}{q}} \lesssim\|f\|_{\mathcal{M}_{q}^{\varphi}} .
$$

The proof of (30) follows from the boundedness of the Hardy-Littlewood maximal operator and the fact that $\varphi(5 r) \simeq \varphi(r)$ for any $r>0$. As for $(31)$, we use

$$
M\left[\chi_{\mathbb{R}^{n} \backslash 5 Q} f\right](y) \lesssim \sup _{R: Q \subset R \in \mathcal{Q}} \frac{1}{|R|} \int_{R}|f(z)| d z \quad(y \in Q)
$$

with $Q=Q(x, r)$. Then by virtue of the fact that $\varphi$ is increasing, we obtain

$$
\begin{aligned}
\varphi(r)\left(\frac{1}{|Q(x, r)|} \int_{Q(x, r)} M f_{2}(y)^{q} d y\right)^{\frac{1}{q}} & \lesssim \varphi(r) \times \sup _{R: Q \subset R \in \mathcal{Q}} \frac{1}{|R|} \int_{R}|f(z)| d z \\
& \lesssim \sup _{R: Q \subset R \in \mathcal{Q}} \varphi(r) \times \frac{1}{|R|} \int_{R}|f(z)| d z \\
& \lesssim\|f\|_{\mathcal{M}_{1}^{\varphi}} \\
& \leq\|f\|_{\mathcal{M}_{q}^{\varphi}},
\end{aligned}
$$

where for the last inequality, we used the nesting property of $\mathcal{M}_{q}^{\varphi}\left(\mathbb{R}^{n}\right), 1 \leq q<$ $\infty$.

As we have seen, any function $\varphi:(0, \infty) \rightarrow(0, \infty)$ will do. So we have the following boundedness for small Morrey spaces.

Example 4.2. Let $1<q \leq p<\infty$. Then $M$ is bounded on $m_{q}^{p}\left(\mathbb{R}^{n}\right)$ and hence $L_{\text {uloc }}^{q}\left(\mathbb{R}^{n}\right)$. In fact, $M$ is bounded on $\mathcal{M}_{q}^{\varphi}\left(\mathbb{R}^{n}\right) \sim m_{q}^{p}\left(\mathbb{R}^{n}\right)$ thanks to Theorem 4.1 , where $\varphi(t)=\max \left(t^{\frac{n}{p}}, 1\right), t>0$.

Similar to Theorem 4.1, we can prove the following theorem:

Theorem 4.3. [73, Theorem 1], [75, Corollary 6.2] Let $1 \leq q<\infty$ and $\varphi \in \mathcal{G}_{q}$. Then $\|M f\|_{\mathrm{w}_{\mathcal{M}}^{\varphi}} \lesssim\|f\|_{\mathcal{M}_{q}^{\varphi}}$ for all measurable functions $f$. 
Once again any function $\mathcal{G}_{q}$ will do as long as $\varphi \in \mathcal{G}_{q}$. Theorem 4.3 extends the result in [10] from Morrey spaces to generalized Morrey spaces.

Proof. Simply resort to the weak- $(1,1)$ boundedness of $M$ and modify the proof of Theorem 4.1.

We move on to the vector-valued inequality. We use the following estimate:

Lemma 4.4. For all measurable functions $f$ and cubes $Q$, we have

$$
M\left[\chi_{\mathbb{R}^{n} \backslash 5 Q} f\right](y) \lesssim \sup _{Q \subset R \in \mathcal{Q}} m_{R}(|f|) \quad(y \in Q) .
$$

Proof. We write out $M\left[\chi_{\mathbb{R}^{n} \backslash 5 Q} f\right](y)$ in full:

$$
M\left[\chi_{\mathbb{R}^{n} \backslash 5 Q} f\right](y)=\sup _{R \in \mathcal{Q}} \frac{\chi_{R}(y)}{|R|}\|f\|_{L^{1}(R \backslash 5 Q)},
$$

where $R$ runs over all cubes. In order that $\chi_{R}(y)\|f\|_{L^{1}(R \backslash 5 Q)}$ be nonzero, we need to have $y \in R$ and $R \backslash 5 Q \neq \emptyset$. Thus, $R$ meets both $Q$ and $\mathbb{R}^{n} \backslash 5 Q$. If $R \in \mathcal{Q}$ is a cube that meets both $Q$ and $\mathbb{R}^{n} \backslash 5 Q$, then $\ell(R) \geq 2 \ell(Q)$ and $2 R \supset Q$. Thus, (32) follows.

Unlike the usual maximal inequality, we need the integral condition (33).

Theorem 4.5. Let $1<q<\infty, 1<u \leq \infty$ and $\varphi \in \mathcal{G}_{q}$. Assume in addition that

$$
\int_{r}^{\infty} \frac{d s}{\varphi(s) s} \lesssim \frac{1}{\varphi(r)} \quad(r>0) .
$$

Then for all $\left\{f_{j}\right\}_{j=1}^{\infty} \subset \mathcal{M}_{q}^{\varphi}\left(\mathbb{R}^{n}\right)$,

$$
\left\|\left(\sum_{j=1}^{\infty} M f_{j}^{u}\right)^{\frac{1}{u}}\right\|_{\mathcal{M}_{q}^{\varphi}} \lesssim\left\|\left(\sum_{j=1}^{\infty}\left|f_{j}\right|^{u}\right)^{\frac{1}{u}}\right\|_{\mathcal{M}_{q}^{\varphi}} .
$$

Proof. When $u=\infty$, the result is clear from Theorem 4.1. The proof is essentially the same as the classical case except that we truly use (33). The proof of the estimate of the inner term remains unchanged except in that we need to generalize the parameter $p$ to the function $\varphi$. Let $f_{j, 1}=\chi_{5 Q} f_{j}$ and $f_{j, 2}=f_{j}-f_{j, 1}$. We can handle $f_{j, 1}$ 's in a standard manner as before. Going through a similar argument to the classical case and using Lemma 4.4, we will have

$$
\left\{\frac{\varphi(\ell(Q))^{q}}{|Q|} \int_{Q}\left(\sum_{j=1}^{\infty} M f_{j, 2}(y)^{u}\right)^{\frac{q}{u}} d y\right\}^{\frac{1}{q}} \lesssim \sum_{k=1}^{\infty} \frac{\varphi(\ell(Q))}{\left|2^{k} Q\right|} \int_{2^{k} Q}\left(\sum_{j=1}^{\infty}\left|f_{j}(z)\right|^{u}\right)^{\frac{1}{u}} d z
$$


If we use the definition of the Morrey norm, we obtain

$$
\left\{\frac{\varphi(\ell(Q))^{q}}{|Q|} \int_{Q}\left(\sum_{j=1}^{\infty} M f_{j, 2}(y)^{u}\right)^{\frac{q}{u}} d y\right\}^{\frac{1}{q}} \lesssim \sum_{k=1}^{\infty} \frac{\varphi(\ell(Q))}{\varphi\left(2^{k} \ell(Q)\right)}\left\|\left(\sum_{j=1}^{\infty}\left|f_{j}\right|^{u}\right)^{\frac{1}{u}}\right\|_{\mathcal{M}_{q}^{\varphi}} .
$$

Since $\varphi \in \mathcal{G}_{q}$, we obtain

$$
\sum_{k=1}^{\infty} \frac{\varphi(\ell(Q))}{\varphi\left(2^{k} \ell(Q)\right)} \lesssim \int_{\ell(Q)}^{\infty} \frac{\varphi(\ell(Q))}{\varphi(t) t} d t
$$

If we use $(33)$ and $\varphi \in \mathcal{G}_{q}$, then we have

$$
\sum_{k=1}^{\infty} \frac{\varphi(\ell(Q))}{\varphi\left(2^{k} \ell(Q)\right)} \lesssim 1
$$

Inserting this estimate into (35), we obtain the counterpart to the classical case.

Since (33) is an important condition, we are interested in its characterization. In fact, we have the following useful one.

Theorem 4.6. Assume that $\varphi:(0, \infty) \rightarrow(0, \infty)$ is an almost increasing function. Then the following are equivalent:

- $\varphi$ satisfies $(33)$.

- There exists $m_{0} \in \mathbb{N}$ such that

$$
\varphi\left(2^{m_{0}} r\right)>2 \varphi(r)
$$

for all $r>0$

Proof. Assume that (33) holds. Let $m_{0}^{\prime}$ be a positive integer such that $\varphi\left(2^{m_{0}^{\prime}-1} r\right) \leq$ $2 \varphi(r)$ for all $r>0$. Thus since $\varphi$ is almost increasing,

$$
\frac{\log m_{0}^{\prime}}{2 \varphi(r)} \leq \int_{r}^{2^{m_{0}^{\prime}-1} r} \frac{d s}{\varphi(s) s} \lesssim \frac{1}{\varphi(r)} .
$$

Since $\varphi(r)>0$, we have an upper bound $M$ for $m_{0}^{\prime}$. Thus if we set $m_{0}=M+1$, we obtain the desired number $m_{0}$.

If (33) holds, then

$$
\int_{r}^{\infty} \frac{d s}{\varphi(s) s}=\sum_{j=1}^{\infty} \int_{2^{m_{0}(j-1)} r}^{2^{m_{0} j} r} \frac{d s}{\varphi(s) s} \lesssim \sum_{j=1}^{\infty} \int_{2^{m_{0}(j-1)} r}^{2^{m_{0} j} r} \frac{d s}{2^{j} \varphi(r) s} \lesssim \frac{1}{\varphi(r)},
$$

as required.

As we have mentioned, we need (33) for the vector-valued maximal inequality. We give an example showing that (33) is absolutely necessary: By no means (33) is artificial as the following proposition shows: 
Proposition 4.7. Let $1<q<\infty, 1<u<\infty$ and $\varphi \in \mathcal{G}_{q}$. Assume in addition that

$$
\left\|\left(\sum_{j=1}^{\infty} M f_{j}{ }^{u}\right)^{\frac{1}{u}}\right\|_{\mathrm{w}_{\mathcal{M}}^{\varphi}} \lesssim\left\|\left(\sum_{j=1}^{\infty}\left|f_{j}\right|^{u}\right)^{\frac{1}{u}}\right\|_{\mathcal{M}_{q}^{\varphi}} .
$$

holds for all sequences of measurable functions $\left\{f_{j}\right\}_{j=1}^{\infty}$. Then (33) holds.

We exclude the case where $u=\infty$, where (37) still holds without (33).

Proof. Assume to the contrary; for all $m \in \mathbb{N} \cap[2, \infty)$, there would exist $r_{m}>0$ such that $\varphi\left(2^{m} r_{m}\right) \leq 2 \varphi\left(r_{m}\right)$ for $r=r_{m}$. Fix $m \in \mathbb{N}$ for the time being. Let us consider $f_{j}=\chi_{[1, j]}(m) \chi_{B\left(2^{j} r_{m}\right) \backslash B\left(2^{j-1} r_{m}\right)}$ for $j \in \mathbb{N}$. Observe

$$
\left\|\left(\sum_{j=1}^{\infty}\left|f_{j}\right|^{u}\right)^{\frac{1}{u}}\right\|_{\mathcal{M}_{q}^{\varphi}}=\left\|\left(\sum_{j=1}^{m} \chi_{B\left(2^{j} r_{m}\right) \backslash B\left(2^{j-1} r_{m}\right)}\right)^{\frac{1}{u}}\right\|_{\mathcal{M}_{q}^{\varphi}} .
$$

As a result,

$$
\left\|\left(\sum_{j=1}^{\infty}\left|f_{j}\right|^{u}\right)^{\frac{1}{u}}\right\|_{\mathcal{M}_{q}^{\varphi}} \leq\left\|\chi_{B\left(2^{m} r_{m}\right)}\right\|_{\mathcal{M}_{q}^{\varphi}} \leq \varphi\left(2^{m} r_{m}\right) \lesssim \varphi\left(r_{m}\right) .
$$

Let $x \in B\left(r_{m}\right)$. For $j>m$, we have $M f_{j}(x)=0$. Meanwhile for $1 \leq j \leq m$, we have

$$
\begin{aligned}
M f_{j}(x) & \geq \frac{1}{\left|B\left(x, 2^{j+1} r_{m}\right)\right|} \int_{B\left(x, 2^{j+1} r_{m}\right)} f_{j}(y) d y \\
& \geq \frac{1}{\left|B\left(x, 2^{j+1} r_{m}\right)\right|} \int_{B\left(2^{j} r_{m}\right)} \chi_{B\left(2^{j} r_{m}\right) \backslash B\left(2^{j-1} r_{m}\right)}(y) d y \\
& =\frac{2^{n}-1}{4^{n}} \geq \frac{1}{4^{n}} .
\end{aligned}
$$

Consequently,

$$
\left\|\left(\sum_{j=1}^{\infty} M f_{j}{ }^{u}\right)^{\frac{1}{u}}\right\|_{\mathrm{w} \mathcal{M}_{q}^{\varphi}} \geq \varphi\left(r_{m}\right) \sum_{j=1}^{m}\left(\frac{1}{4^{n}}\right)^{u} \sim \varphi\left(r_{m}\right) m^{\frac{1}{u}} .
$$

By our assumption, we have

$$
\varphi\left(r_{m}\right) m^{\frac{1}{u}} \lesssim\left\|\left(\sum_{j=1}^{\infty} M f_{j}^{u}\right)^{\frac{1}{u}}\right\|_{\mathrm{w}_{\mathcal{M}}^{\varphi}} \lesssim\left\|\left(\sum_{j=1}^{\infty}\left|f_{j}\right|^{u}\right)^{\frac{1}{u}}\right\|_{\mathcal{M}_{q}^{\varphi}} \lesssim \varphi\left(r_{m}\right),
$$

or equivalently

$$
m \leq D
$$


where $D$ does not depend on $m$. This contradicts to the fact that $m \in \mathbb{N} \cap[2, \infty)$ is arbitrary.

Example 4.8. Let $1 \leq q \leq p<\infty$. Then

$$
\left\|\left(\sum_{j=1}^{\infty} M f_{j}^{u}\right)^{\frac{1}{u}}\right\|_{\mathrm{w} m_{q}^{p}} \lesssim\left\|\left(\sum_{j=1}^{\infty}\left|f_{j}\right|^{u}\right)^{\frac{1}{u}}\right\|_{m_{q}^{p}} \quad\left(\left\{f_{j}\right\}_{j=1}^{\infty} \subset m_{q}^{p}\left(\mathbb{R}^{n}\right)\right)
$$

fails. In particular,

$$
\left\|\left(\sum_{j=1}^{\infty} M f_{j}{ }^{u}\right)^{\frac{1}{u}}\right\|_{\mathrm{w} L_{\text {uloc }}^{q}} \lesssim\left\|\left(\sum_{j=1}^{\infty}\left|f_{j}\right|^{u}\right)^{\frac{1}{u}}\right\|_{L_{\text {uloc }}^{q}}\left(\left\{f_{j}\right\}_{j=1}^{\infty} \subset L_{\text {uloc }}^{q}\left(\mathbb{R}^{n}\right)\right)
$$

fails. In fact, let $\varphi(t) \equiv \max \left(t^{\frac{n}{p}}, 1\right)$ for $t>0$ as before. Then $\varphi$ fails (33) because $\int_{u}^{\infty} \frac{d r}{\varphi(r)} \sim \log \frac{1}{u}$.

Proposition 4.7 led us to the conclusion that (33) is fundamental. The following proposition will be fundamental in the study of the boundedness of the operators in generalized Morrey spaces.

Theorem 4.9. [73, Lemma 2] If a nonnegative locally integrable function $\psi$ and a positive constant $D>0$ satisfy

$$
\int_{r}^{\infty} \psi(t) \frac{d t}{t} \leq D \psi(r) \quad(r>0)
$$

then

$$
\int_{r}^{\infty} \psi(t) t^{\varepsilon} \frac{d t}{t} \leq \frac{r^{\varepsilon}}{1-D \varepsilon} \cdot \int_{r}^{\infty} \psi(t) t^{-1} d t \leq \frac{D}{1-D \varepsilon} \cdot \psi(r) r^{\varepsilon} \quad(r>0)
$$

for all $0<\varepsilon<D^{-1}$.

Proof. Let

For $0<r<R$

$$
\Psi(r)=\int_{r}^{\infty} \psi(t) t^{-1} d t \quad(r>0)
$$

$$
\int_{r}^{R} \psi(t) t^{\varepsilon} \frac{d t}{t}=\left[-\Psi(t) t^{\varepsilon}\right]_{r}^{R}+\int_{r}^{R} \Psi(t) \varepsilon t^{\varepsilon} \frac{d t}{t} \leq \Psi(r) r^{\varepsilon}+\varepsilon D \int_{r}^{R} \psi(t) t^{\varepsilon} \frac{d t}{t} .
$$

Therefore

It remains to let $R \rightarrow \infty$.

$$
\int_{r}^{R} \psi(t) t^{\varepsilon} \frac{d t}{t} \leq \frac{1}{1-\varepsilon D} \Psi(r) r^{\varepsilon} \leq \frac{D}{1-\varepsilon D} \psi(r) r^{\varepsilon} .
$$

We change variables to have the following variant: 
Theorem 4.10. [73, Lemma 2] Let $\psi:(0, \infty) \rightarrow(0, \infty)$ be a measurable function satisfying

$$
\int_{0}^{r} \psi(t) \frac{d t}{t} \leq D \psi(r) \quad(r>0)
$$

for some $D>0$ independent of $r>0$. If $0<\varepsilon<D^{-1}$, then

$$
\int_{0}^{r} \psi(t) \frac{d t}{t^{1+\varepsilon}} \leq \frac{1}{1-D \varepsilon} r^{-\varepsilon} \int_{0}^{r} \psi(t) \frac{d t}{t} \leq \frac{D}{1-D \varepsilon} r^{-\varepsilon} \psi(r) .
$$

Proof. Set

$$
\eta(t)=\psi\left(\frac{1}{t}\right) \quad(t>0)
$$

Then our assumption reads as:

$$
\int_{r}^{\infty} \eta(t) \frac{d t}{t} \leq D \eta(r) \quad(r>0)
$$

Thus,

$$
\int_{r}^{\infty} \eta(t) \frac{d t}{t^{1-\varepsilon}} \leq \frac{1}{1-D \varepsilon} r^{\varepsilon} \int_{r}^{\infty} \eta(t) \frac{d t}{t} \leq \frac{D}{1-D \varepsilon} r^{\varepsilon} \eta(r) \quad(r>0)
$$

according to Theorem 4.9. If we express this inequality in terms of $\psi$, we obtain the desired result. (33).

In the next proposition, we further characterize and apply our key assumption

Proposition 4.11. [80, Proposition 2.7] Let $\varphi$ be a nonnegative locally integrable function such that $\varphi(s) \lesssim \varphi(r)$ for all $r, s>0$ with $\frac{1}{2} \leq \frac{r}{s} \leq 2$. There exists a constant $\varepsilon>0$ such that

$$
\frac{t^{\varepsilon}}{\varphi(t)} \lesssim \frac{r^{\varepsilon}}{\varphi(r)} \quad(t \geq r)
$$

holds if and only if holds (33), or equivalently, $\varphi$ satisfies (38) for some $\varepsilon>0$. If one of these conditions is satisfied, then

$$
\int_{r}^{\infty} \frac{d s}{\varphi(s)^{u} s} \lesssim \frac{1}{\varphi(r)^{u}} \quad(r>0)
$$

for all $0<u<\infty$, where the implicit constant depends only on $u$.

Proof. The implication (33) $\Longrightarrow$ (38) follows from Proposition 4.9.

Assume (38). Then we have

$$
\frac{t^{\varepsilon}}{\varphi(t)} \lesssim \int_{t}^{2 t} \frac{d v}{v^{1-\varepsilon} \varphi(v)} \lesssim \frac{r^{\varepsilon}}{\varphi(r)}
$$

thanks to the doubling property of $\varphi$, proving (39).

If we assume (39), then we have

$$
\int_{r}^{\infty} \frac{d s}{\varphi(s) s}=\int_{r}^{\infty} \frac{s^{\varepsilon}}{\varphi(s)} \frac{d s}{s^{1+\varepsilon}} \lesssim \int_{r}^{\infty} \frac{r^{\varepsilon}}{\varphi(r)} \frac{d s}{s^{1+\varepsilon}}=\frac{1}{\varepsilon \varphi(r)},
$$


which implies (33). Note that (39) also implies (40) because $\varphi^{u}$ satisfies (39) as well.

Let $0<u<\infty$ be fixed. Inequality (40) is necessary for (33); simply apply Proposition 4.11 to $\varphi^{u}$

Example 4.12. Let $\varphi \in \mathcal{G}_{q}$ satisfy (40). Then we have

$$
\int_{r}^{\infty} \frac{1}{\varphi(s) s^{1-\varepsilon}} d s \lesssim \frac{r^{\varepsilon}}{\varphi(r)} \quad(r>0)
$$

Hence

$$
\frac{s^{\varepsilon}}{\varphi(s)} \lesssim \frac{r^{\varepsilon}}{\varphi(r)}
$$

whenever $0<r \leq s<\infty$. As a result,

$$
\int_{0}^{1} \varphi(r) \frac{d r}{r}<\infty
$$

Let $\varphi(t) \equiv \max \left(t^{\frac{n}{p}}, 1\right)$ for $t>0$ as before. Then $\varphi$ clearly fails to satisfy this condition.

We generalize condition (33) as follows:

Definition 4.13. Let $\gamma \in \mathbb{R}$.

- The (upper) Zygmund class $\mathbb{Z}^{\gamma}$ is defined to be the set of all measurable functions $\varphi:(0, \infty) \rightarrow(0, \infty)$ for which $\lim _{r \downarrow 0} \varphi(r)=0$ and

$$
\int_{0}^{r} \varphi(t) t^{-\gamma-1} d t \lesssim \varphi(r) r^{-\gamma} \quad(r>0)
$$

- The (lower) Zygmund class $\mathbb{Z}_{\gamma}$ is defined to be the set of all measurable functions $\varphi:(0, \infty) \rightarrow(0, \infty)$ for which $\lim _{r \downarrow 0} \varphi(r)=0$ and

$$
\int_{r}^{\infty} \varphi(t) t^{-\gamma-1} d t \lesssim \varphi(r) r^{-\gamma} \quad(r>0) .
$$

Note that (33) reads as $\frac{1}{\varphi} \in \mathbb{Z}_{0}$.

Example 4.14. Let $\varphi(t)=t^{p}$ with $1<p<\infty$, and let $\gamma \in \mathbb{R}$.

- $\varphi \in \mathbb{Z}^{\gamma}$ if and only if $p>\gamma$.

- $\varphi \in \mathbb{Z}_{\gamma}$ if and only if $p<\gamma$.

- $1 \notin \mathbb{Z}^{\gamma}$ if and only if $\gamma<0$.

We present an example of the functions in $\mathcal{M}_{q}^{\varphi}\left(\mathbb{R}^{n}\right)$. Let $0<\eta<\infty$. We define the powered Hardy-Littlewood maximal operator $M^{(\eta)}$ by

$$
M^{(\eta)} f(x) \equiv \sup _{R>0}\left(\frac{1}{|B(x, R)|} \int_{B(x, R)}|f(y)|^{\eta} \mathrm{d} y\right)^{\frac{1}{\eta}} \quad\left(x \in \mathbb{R}^{n}\right) .
$$


Example 4.15. [80, Proposition 2.11] Let $0<q<\infty$ and $\varphi \in \mathcal{G}_{q}$. Define

$$
f \equiv \sum_{j=-\infty}^{\infty} \frac{\chi_{\left[2^{-j-1}, 2^{-j}\right]^{n}}}{\varphi\left(2^{-j}\right)}, \quad g \equiv \sup _{j \in \mathbb{Z}} \frac{\chi_{\left[0,2^{-j}\right]^{n}}}{\varphi\left(2^{-j}\right)}
$$

We claim that the following are equivalent;
(a) $f \in \mathcal{M}_{q}^{\varphi}\left(\mathbb{R}^{n}\right)$,
(b) $g \in \mathcal{M}_{q}^{\varphi}\left(\mathbb{R}^{n}\right)$,
(c) $\frac{1}{\varphi} \in \mathbb{Z}^{\frac{n}{q}}$.

Let $0<u<q$. Note that $f \leq g \leq 2^{n} M^{(u)} f$, where $M^{(u)}$ denotes the powered Hardy-Littlewood maximal operator. Observe that $M^{(u)}$ is bounded on $\mathcal{M}_{q}^{\varphi}\left(\mathbb{R}^{n}\right)$. Thus $(a)$ and $(b)$ are equivalent. Since $f$ is expressed as $f=f_{0}\left(\|\cdot\|_{\infty}\right)$, that is, there exists a function $f_{0}:[0, \infty) \rightarrow \mathbb{R}$ such that $f(x)=f_{0}\left(\|x\|_{\infty}\right)$ for all $x \in \mathbb{R}^{n}$, where $\|\cdot\|_{\infty}$ denotes the $\ell^{\infty}$-norm, it follows that $(a)$ and $(c)$ are equivalent.

Example 4.16. [80, Proposition 2.11] Let $0<q<\infty$, and let $\varphi \in \mathcal{G}_{q}$. Define a decreasing function $\varphi^{\dagger}$ by:

$$
\varphi^{\dagger}(t) \equiv \varphi(t) t^{-\frac{n}{q}}
$$

for $t>0$. Define $h \equiv \sum_{j=-\infty}^{\infty} \frac{\chi_{\left[0,2^{-j}\right]^{n}}}{\varphi\left(2^{-j}\right)}$. Then $\sum_{j=l}^{\infty} \frac{1}{\varphi\left(2^{-j}\right)} \lesssim \frac{1}{\varphi\left(2^{-l}\right)}$ and that

$$
\sum_{j=\infty}^{l} \frac{1}{\varphi^{\dagger}\left(2^{-j}\right)} \lesssim \frac{1}{\varphi^{\dagger}\left(2^{-l}\right)}
$$

for all $l \in \mathbb{Z}$ if and only if $h \in \mathcal{M}_{q}^{\varphi}\left(\mathbb{R}^{n}\right)$.

To verify this, we let $f, g$ be as in Example 4.15. Suppose first $h \in \mathcal{M}_{q}^{\varphi}\left(\mathbb{R}^{n}\right)$. Then

$$
\varphi\left(2^{-l}\right)\left(\frac{1}{\left|\left[0,2^{-l}\right]^{n}\right|} \int_{\left[0,2^{-l}\right]^{n}}\left(\sum_{j=l}^{\infty} \frac{1}{\varphi\left(2^{-j}\right)}\right)^{q} d x\right)^{\frac{1}{q}} \leq\|h\|_{\mathcal{M}_{q}^{\varphi}}
$$

Thus $\sum_{j=l}^{\infty} \frac{1}{\varphi\left(2^{-j}\right)} \leq \frac{\|h\|_{\mathcal{M}_{q}^{\varphi}}}{\varphi\left(2^{-l}\right)}$ for all $l \in \mathbb{Z}$. This implies that $f \leq g \leq h \lesssim f$, where $f$ and $g$ are defined in Example 4.15. Thus from Example 4.15, $\sum_{j=\infty}^{l} \frac{1}{\varphi^{\dagger}\left(2^{-j}\right)} \lesssim$ $\frac{1}{\varphi^{\dagger}\left(2^{-l}\right)}$ holds as well.

Conversely, assume that

$$
\sum_{j=\infty}^{l} \frac{1}{\varphi^{\dagger}\left(2^{-j}\right)} \lesssim \frac{1}{\varphi^{\dagger}\left(2^{-l}\right)}
$$


and

$$
\sum_{j=l}^{\infty} \frac{1}{\varphi\left(2^{-j}\right)} \lesssim \frac{1}{\varphi\left(2^{-l}\right)}
$$

hold for all $l \in \mathbb{Z}$. Then we have $h \sim f$ from (43). Thus $f \in \mathcal{M}_{q}^{\varphi}\left(\mathbb{R}^{n}\right)$ by (42), from which it follows that $h \in \mathcal{M}_{q}^{\varphi}\left(\mathbb{R}^{n}\right)$.

We further present some examples of the functions in $\mathcal{M}_{p}^{\varphi}\left(\mathbb{R}^{n}\right)$.

Lemma 4.17. [18, Lemma 2.4] Let $0<q<\infty$ and $\varphi \in \mathcal{G}_{q} \cap \mathbb{Z}^{-\frac{n}{q}}$. Then the function $\psi(x)=\varphi(|x|)$ belongs to $\mathcal{M}_{q}^{\varphi}\left(\mathbb{R}^{n}\right)$.

Proof. First note that $\varphi \in \mathbb{Z}^{-\frac{n}{q}}$ is equivalent to

$$
\frac{1}{r^{n}} \int_{0}^{r} \varphi(t)^{q} t^{n-1} d t \lesssim \varphi(r)^{q} \quad(r>0) .
$$

Note that $\varphi(|\cdot|)$ is radial decreasing, so that for all $a \in \mathbb{R}^{n}$ and $r>0$,

$$
\left(\frac{1}{|B(a, r)|} \int_{B(a, r)} \varphi(|x|)^{q} d x\right)^{\frac{1}{q}} \leq\left(\frac{1}{|B(r)|} \int_{B(r)} \varphi(|x|)^{q} d x\right)^{\frac{1}{q}} .
$$

Combining (44) and (45) and using the spherical coordinate, we obtain the desired result.

Remark 4.18. See [110, Theorem 2.1] for the weak boundedness of the maximal operators, where the integral conditions is assumed.

Remark 4.19. See [29, Theorem 4.2] and [2, Theorem 3.4] for the strong boundedness of the maximal operators, where the integral conditions is assumed. See $[79,75,91]$ for the strong boundedness of the maximal operators, where the integral conditions is not assumed.

Remark 4.20. See [7, Theorem 1], [108, Theorem 2.9], [120, Theorem 1.9] and [127, Theorem 2.1] for the boundedness of the maximal operator on generalized Morrey spaces in the multilinear setting.

4.2. Singular integral operators on generalized Morrey spaces. Let $T$ be a singular integral operator. To define the function $T f$ for $f \in \mathcal{M}_{q}^{\varphi}\left(\mathbb{R}^{n}\right)$ we follow the same strategy as the one for $f \in \mathcal{M}_{q}^{p}\left(\mathbb{R}^{n}\right)$. To this end, we need to establish the following estimate:

Lemma 4.21. Let $1<q<\infty$ and $\varphi \in \mathcal{G}_{q}$ satisfy $\frac{1}{\varphi} \in \mathbb{Z}_{0}$. Then $\|T f\|_{\mathcal{M}_{q}^{\varphi}} \lesssim\|f\|_{\mathcal{M}_{q}^{\varphi}}$ for all $f \in L_{\mathrm{c}}^{\infty}\left(\mathbb{R}^{n}\right)$.

We note that $\frac{1}{\varphi} \in \mathbb{Z}_{0}$ appeared once again.

Proof. Let $Q$ be a fixed cube. Then we need to prove

$$
\varphi(\ell(Q))\left(\frac{1}{|Q|} \int_{Q}|T f(y)|^{q} d y\right)^{\frac{1}{q}} \lesssim\|f\|_{\mathcal{M}_{q}^{\varphi}} .
$$


To this end, we decompose $f$ according to $2 Q: f_{1}=\chi_{2 Q} f, f_{2}=f-f_{1}$. As for $f_{1}$, we have

$$
\begin{aligned}
\varphi(\ell(Q))\left(\frac{1}{|Q|} \int_{Q}\left|T f_{1}(y)\right|^{q} d y\right)^{\frac{1}{q}} & \leq \varphi(\ell(Q))\left(\frac{1}{|Q|} \int_{\mathbb{R}^{n}}\left|T f_{1}(y)\right|^{q} d y\right)^{\frac{1}{q}} \\
& \lesssim \varphi(\ell(Q))\left(\frac{1}{|Q|} \int_{\mathbb{R}^{n}}\left|f_{1}(y)\right|^{q} d y\right)^{\frac{1}{q}} \\
& \lesssim \varphi(\ell(Q))\left(\frac{1}{|Q|} \int_{2 Q}|f(y)|^{q} d y\right)^{\frac{1}{q}} \\
& \lesssim\|f\|_{\mathcal{M}_{q}^{\varphi}} .
\end{aligned}
$$

As for $f_{2}$, we use the size condition of $K$, the integral kernel of $T$, to have the local estimate:

$$
\left|T f_{2}(y)\right| \lesssim \int_{\mathbb{R}^{n} \backslash 2 Q} \frac{|f(y)| d y}{|y-c(Q)|^{n}} \lesssim \int_{\ell(Q)}^{\infty}\left(\frac{1}{\ell^{n+1}} \int_{B(c(Q), \ell)}|f(y)| d y\right) d \ell .
$$

By the definition of the norm, Lemma 3.12 and (33), we obtain

$$
\left|T f_{2}(y)\right| \lesssim \int_{\ell(Q)}^{\infty} \frac{1}{r \varphi(r)} d r \cdot\|f\|_{\mathcal{M}_{q}^{\varphi}} \lesssim \frac{1}{\varphi(\ell(Q))}\|f\|_{\mathcal{M}_{q}^{\varphi}}
$$

It remains to integrate this pointwise estimate.

To carry out our program of proving the boundedness of the singular integral operators, we need to investigate the predual and its predual.

Definition 4.22. Let $1<q<\infty$ and $\varphi \in \mathcal{G}_{q}$.

- [60, Definition 2.3], [109, Definition 4] $A(\varphi, q)$-block is said to be a measurable function A supported on a cube $Q$ satisfying $\|A\|_{L^{q^{\prime}}} \leq|Q|^{-\frac{1}{q}} \varphi(\ell(Q))$. In this case $A$ is said to be a $(\varphi, q)$-block supported on $Q$.

- [60, Definition 2.5], [109, Definition 5] The block space $\mathcal{H}_{q^{\prime}}^{\varphi}\left(\mathbb{R}^{n}\right)$ is the set of all measurable functions $f$ for which it can be written

$$
f=\sum_{j=1}^{\infty} \lambda_{j} A_{j}
$$

for some sequence $\left\{A_{j}\right\}_{j=1}^{\infty}$ of $(\varphi, q)$-blocks and $\left\{\lambda_{j}\right\}_{j=1}^{\infty} \in \ell^{1}\left(\mathbb{R}^{n}\right)$. The norm $\|f\|_{\mathcal{H}_{q^{\prime}}^{\varphi}}$ is the infimum of $\left\|\left\{\lambda_{j}\right\}_{j=1}^{\infty}\right\|_{\ell^{1}}$ where $\left\{A_{j}\right\}_{j=1}^{\infty}$ and $\left\{\lambda_{j}\right\}_{j=1}^{\infty}$ run over all expressions above.

Example 4.23. Let $1<q<\infty$ and $\varphi \in \mathcal{G}_{q}$. Let $A$ be a non-zero $L^{q^{\prime}}\left(\mathbb{R}^{n}\right)$-function supported on a cube $Q$. Then $B \equiv \frac{\varphi(\ell(Q))}{|Q|^{\frac{1}{q}}\|A\|_{L^{q^{\prime}}}} A$ is a $(\varphi, q)$-block supported on $Q$. 
Proposition 4.24. Let $1<q<\infty$ and $\varphi \in \mathcal{G}_{q}$ be such that $\varphi(t) \gtrsim t^{\frac{n}{q}}$ for alll $t>0$. Then a measurable function $f$ belongs to $\mathcal{H}_{q^{\prime}}^{\varphi}\left(\mathbb{R}^{n}\right)$ if and only if $f$ admits a decomposition:

$$
f=\lambda_{0} B+\sum_{j=1}^{\infty} \lambda_{j} A_{j}
$$

for some sequence $\left\{A_{j}\right\}_{j=1}^{\infty}$ of $(\varphi, q)$-blocks supported on cubes of volume less than or equal to 1 and $\left\{\lambda_{j}\right\}_{j=1}^{\infty} \in \ell^{1}\left(\mathbb{R}^{n}\right)$ and $B \in L^{q^{\prime}}\left(\mathbb{R}^{n}\right)$ with unit norm. Furthermore the norm $\|f\|_{\mathcal{H}_{q^{\prime}}}$ is the infimum of $\left\|\left\{\lambda_{j}\right\}_{j=0}^{\infty}\right\|_{\ell^{1}}$ where $\left\{A_{j}\right\}_{j=1}^{\infty}, B$ and $\left\{\lambda_{j}\right\}_{j=1}^{\infty}$ run over all expressions above.

Proof. Let $A$ be a $(\varphi, q)$-block supported on $Q$ with $\ell(Q) \gg 1$. Then we can say that $A$ is a $(\varphi, q)$-block suppported on $Q$ is and only if $2 A$ has the $L^{q^{\prime}}\left(\mathbb{R}^{n}\right)$-norm 1 and $A$ is supported on $Q$. So, in the decomposition in Definition 4.22 any block $A_{j}$ with the cube $Q_{j}$ satisfying $\left|Q_{j}\right| \gg 1$ can be combined into a block supported on " $\mathbb{R}^{n}$ ".

The following lemma justifies the definition above.

Lemma 4.25. [109, Lemma 2] Let $1<q<\infty$ and $\varphi \in \mathcal{G}_{q}$. If $A$ is a $(\varphi, q)$-block and $f \in \mathcal{M}_{q}^{\varphi}\left(\mathbb{R}^{n}\right)$, then $\|A \cdot f\|_{L^{1}} \leq\|f\|_{\mathcal{M}_{q}^{\varphi}}$.

Proof. Since $A$ is a $(\varphi, q)$-block, we can find a cube $Q$ such that $\operatorname{supp}(A) \subset Q$ and that $\|A\|_{L^{q^{\prime}}} \leq|Q|^{-\frac{1}{q}} \varphi(\ell(Q))$. By the Hölder inequality,

$$
\|A \cdot f\|_{L^{1}}=\left\|A \cdot f \chi_{Q}\right\|_{L^{1}} \leq\|A\|_{L^{q^{\prime}}}\left\|f \chi_{Q}\right\|_{L^{q}} \leq|Q|^{-\frac{1}{q}} \varphi(\ell(Q))\left\|f \chi_{Q}\right\|_{L^{q}} \leq\|f\|_{\mathcal{M}_{q}^{\varphi}},
$$

as required.

About the definition above, the following proposition is fundamental:

Proposition 4.26. [60, Lemma 4.2] Let $1<q<\infty$ and $\varphi \in \mathcal{G}_{q}$. Let $f \in \mathcal{M}_{q}^{\varphi}\left(\mathbb{R}^{n}\right)$ and $g \in \mathcal{H}_{q^{\prime}}^{\varphi}\left(\mathbb{R}^{n}\right)$. Then $\|f \cdot g\|_{L^{1}} \leq\|f\|_{\mathcal{M}_{q}^{\varphi}}\|g\|_{\mathcal{H}_{q^{\prime}}^{\varphi}}$.

Proof. Let $\varepsilon>0$ be fixed. Then we can decompose $g$ as

$$
g=\sum_{k \in K} \lambda_{k} b_{k}
$$

where $K \subset \mathbb{N}$ is an index set,

$$
\sum_{k \in K}\left|\lambda_{k}\right| \leq(1+\varepsilon)\|g\|_{\mathcal{H}_{q^{\prime}}^{\varphi}}
$$

and each $b_{k}$ is a $(\varphi, q)$-block. According to Lemma 4.25, we have

$$
\|f \cdot g\|_{L^{1}} \leq \sum_{k \in K}\left|\lambda_{k}\right| \cdot\|f\|_{\mathcal{M}_{q}^{\varphi}} \leq(1+\varepsilon)\|f\|_{\mathcal{M}_{q}^{\varphi}}\|g\|_{\mathcal{H}_{q^{\prime}}^{\varphi}} .
$$

Consequently

$$
\|f \cdot g\|_{L^{1}} \leq(1+\varepsilon)\|f\|_{\mathcal{M}_{q}^{\varphi}}\|g\|_{\mathcal{H}_{q^{\prime}}^{\varphi}}
$$


Since $\varepsilon>0$ is arbitrary, it follows that

$$
\|f \cdot g\|_{L^{1}} \leq\|f\|_{\mathcal{M}_{q}^{\varphi}}\|g\|_{\mathcal{H}_{q^{\prime}}^{\varphi}} .
$$

For $j \in \mathbb{Z}$ and $k=\left(k_{1}, k_{2}, \ldots, k_{n}\right) \in \mathbb{Z}^{n}$ define the set $Q_{j k}$ by

$$
Q_{j k} \equiv\left[\frac{k_{1}}{2^{j}}, \frac{k_{1}+1}{2^{j}}\right) \times \cdots \times\left[\frac{k_{n}}{2^{j}}, \frac{k_{n}+1}{2^{j}}\right)=\prod_{l=1}^{n}\left[\frac{k_{l}}{2^{j}}, \frac{k_{l}+1}{2^{j}}\right) .
$$

A dyadic cube is a set of the form $Q_{j k}$ for some $j \in \mathbb{Z}, k=\left(k_{1}, k_{2}, \ldots, k_{n}\right) \in \mathbb{Z}^{n}$. The set of all dyadic cubes is denoted by $\mathcal{D} ; \mathcal{D}=\mathcal{D} \equiv\left\{Q_{j k}: j \in \mathbb{Z}, k \in \mathbb{Z}^{n}\right\}$. For $j \in \mathbb{Z}$ the set of dyadic cubes of the $j$-th generation is given by

$$
\mathcal{D}_{j}=\mathcal{D}_{j}\left(\mathbb{R}^{n}\right) \equiv\left\{Q_{j k}: k \in \mathbb{Z}^{n}\right\}=\left\{Q \in \mathcal{D}: \ell(Q)=2^{-j}\right\} .
$$

It is easy to see that $\mathcal{H}_{q^{\prime}}^{\varphi}\left(\mathbb{R}^{n}\right)$ is a normed space. Similar to the classical case, we can prove the following decomposition theorem:

Theorem 4.27. Let $1<q<\infty, \varphi \in \mathcal{G}_{q}$, and let $f \in \mathcal{H}_{q^{\prime}}^{\varphi}\left(\mathbb{R}^{n}\right)$. Then $f$ can be decomposed as

$$
f=\sum_{Q \in \mathcal{D}} \lambda(Q) b(Q)
$$

where $\lambda(Q)$ is a non-negative number with

$$
\sum_{Q \in \mathcal{D}} \lambda(Q) \leq 3^{n}\|f\|_{\mathcal{H}_{q^{\prime}}^{\varphi}}
$$

and $b(Q)$ is a $(\varphi, q)$-block supported in $Q$.

Proof. We suppose that $\|f\|_{\mathcal{H}_{q^{\prime}}^{\varphi}}<1$. It suffices to find a decomposition

$$
f=\sum_{Q \in \mathcal{D}} \lambda(Q) b(Q)
$$

where $\lambda(Q)$ is a non-negative number with

$$
\sum_{Q \in \mathcal{D}} \lambda(Q)<3^{n}
$$

First, decompose $f$ as

$$
f=\sum_{k \in K} \lambda_{k} b_{k}
$$

where $K \subset \mathbb{N}$ is an index set,

$$
\sum_{k \in K}\left|\lambda_{k}\right|<1
$$

and each $b_{k}$ is a $(\varphi, q)$-block. We will divide $K$ into the $\operatorname{disjoint}$ sets $K(Q) \subset \mathbb{N}$, $Q \in \mathcal{D}$, as

$$
K=\bigcup_{Q \in \mathcal{D}} K(Q)
$$


so that $\operatorname{supp}\left(b_{k}\right) \subset 3 Q$ and $\left|Q_{k}\right| \geq|Q|$ whenever $k \in K(Q)$. We achieve this as follows: Let $\mathcal{D}=\left\{Q^{(j)}\right\}_{j=1}^{\infty}$ be an emumeration of $\mathcal{D}$. For each $k \in K$, we write

$$
j_{k} \equiv \min \left\{j: \operatorname{supp}\left(b_{k}\right) \subset 3 Q^{(j)},\left|Q_{k}\right| \geq\left|Q^{(j)}\right|\right\}
$$

for each $k$. We set

$$
K\left(Q^{(j)}\right) \equiv\left\{k \in K: j_{k}=j\right\} .
$$

We set

$$
\lambda(Q) \equiv 3^{n} \sum_{k \in K(Q)}\left|\lambda_{k}\right|, \quad b(Q) \equiv \begin{cases}\frac{1}{\lambda(Q)} \sum_{k \in K(Q)} \lambda_{k} b_{k} & (\lambda(Q) \neq 0), \\ 0 & (\lambda(Q) \neq 0) .\end{cases}
$$

We now rewrite $f$ as

$$
\begin{aligned}
f & =\sum_{k \in K} \lambda_{k} b_{k}=\sum_{Q \in \mathcal{D}}\left(\sum_{k \in K(Q)} \lambda_{k} b_{k}\right) \\
& =\sum_{Q \in \mathcal{D}}\left\{3^{n} \sum_{k \in K(Q)}\left|\lambda_{k}\right|\right\} \cdot\left\{\left(3^{n} \sum_{k \in K(Q)}\left|\lambda_{k}\right|\right)^{-1} \sum_{Q \in \mathcal{D}} \lambda(Q) b(Q)\right\} .
\end{aligned}
$$

By (47), we have

$$
\sum_{Q \in \mathcal{D}} \lambda(Q)=3^{n} \sum_{Q \in \mathcal{D}}\left(\sum_{k \in K(Q)}\left|\lambda_{k}\right|\right)=3^{n} \sum_{k \in K}\left|\lambda_{k}\right|<3^{n} .
$$

Since each $b_{k}$ is a $(\varphi, q)$-block, we obtain

$$
\begin{aligned}
\left(3^{n} \sum_{k \in K(Q)}\left|\lambda_{k}\right|\right)^{-1}\left\|\sum_{k \in K(Q)} \lambda_{k} b_{k}\right\|_{q^{\prime}} & \leq\left(3^{n} \sum_{k \in K(Q)}\left|\lambda_{k}\right|\right)^{-1} \sum_{k \in K(Q)}\left|\lambda_{k}\right| \cdot\left\|b_{k}\right\|_{q^{\prime}} \\
& \leq\left(3^{n} \sum_{k \in K(Q)}\left|\lambda_{k}\right|\right)^{-1} \varphi(\ell(Q))|Q|^{-\frac{1}{q}} \sum_{k \in K(Q)}\left|\lambda_{k}\right| \\
& \leq \varphi(\ell(3 Q))|3 Q|^{-\frac{1}{q}},
\end{aligned}
$$

which implies that $b(Q)$ is a $(\varphi, q)$-block supported in $3 Q$. These complete the proof.

Example 4.28. Let $1<q<\infty, \varphi \in \mathcal{G}_{q}$, and let $f \in \mathcal{H}_{q^{\prime}}^{\varphi}\left(\mathbb{R}^{n}\right)$. Assume that $\inf _{t>0} t^{-\frac{n}{q}} \varphi(t)>0$, so that Proposition 4.24 is applicable. Then $f$ can be decomposed as

$$
f=B+\sum_{Q \in \mathcal{D},|Q| \leq 1} \lambda(Q) b(Q),
$$


where $\lambda(Q)$ is a non-negative number with

$$
\|B\|_{L^{q^{\prime}}}+\sum_{Q \in \mathcal{D}} \lambda(Q) \lesssim\|f\|_{\mathcal{H}_{q^{\prime}}^{\varphi}}
$$

and $b(Q)$ is a $(\varphi, q)$-block supported in $Q$.

Corollary 4.29. Let $1<q<\infty$ and $\varphi \in \mathcal{G}_{q}$. Then every function in $\mathcal{H}_{q^{\prime}}^{\varphi}\left(\mathbb{R}^{n}\right)$ is locally integrable.

Proof. Simply combine Lemma 3.12, Proposition 4.26 and the fact that $\chi_{Q} \in$ $\mathcal{M}_{q}^{\varphi}\left(\mathbb{R}^{n}\right)$ for any cube $Q$.

Proposition 4.30. Let $1<q<\infty$ and $\varphi \in \mathcal{G}_{q}$. Assume in addition that $\varphi$ satisfies (33). Suppose that $f$ and $f_{k},(k=1,2, \ldots)$, are nonnegative, that each $f_{k} \in \mathcal{H}_{q^{\prime}}^{\varphi^{\prime}}\left(\mathbb{R}^{n}\right)$, that $\left\|f_{k}\right\|_{\mathcal{H}_{q^{\prime}}^{\varphi^{\prime}}} \leq 1$ and that $f_{k} \uparrow f$ a.e. Then $f \in \mathcal{H}_{q^{\prime}}^{\varphi^{\prime}}\left(\mathbb{R}^{n}\right)$ and $\|f\|_{\mathcal{H}_{q^{\prime}}^{\varphi^{\prime}}} \leq 1$.

Proof. By Theorem $4.27 f_{k}$ can be decomposed as

$$
f_{k}=\sum_{Q \in \mathcal{D}} \lambda_{k}(Q) b_{k}(Q)
$$

where $\lambda_{k}(Q)$ is a non-negative number with

$$
\sum_{Q \in \mathcal{D}} \lambda_{k}(Q) \leq 3^{n}
$$

and $b_{k}(Q)$ is a $(\varphi, q)$-block supported in $Q$ and

$$
\left\|b_{k}(Q)\right\|_{q^{\prime}} \leq \frac{\varphi(\ell(Q))}{|Q|^{\frac{1}{q}}} .
$$

Using (48), (49) and the weak-compactness of the Lebesgue space $L^{q^{\prime}}(Q)$ we now apply a diagonalization argument and, hence, we can select an increasing sequence $\left\{k_{j}\right\}_{j=1}^{\infty}$ of integers that satisfies the following:

$$
\begin{gathered}
\lim _{j \rightarrow \infty} \lambda_{k_{j}}(Q)=\lambda(Q), \\
\lim _{j \rightarrow \infty} b_{k_{j}}(Q)=b(Q) \text { in the weak-topology of } L^{q^{\prime}}(Q),
\end{gathered}
$$

where $b(Q)$ is a $(\varphi, q)$-block supported in $Q$. We set

$$
f_{0} \equiv \sum_{Q \in \mathcal{D}} \lambda(Q) b(Q) .
$$

Then, by the Fatou theorem and (48),

$$
\sum_{Q \in \mathcal{D}} \lambda(Q) \leq \liminf _{j \rightarrow \infty} \sum_{Q \in \mathcal{D}} \lambda_{k_{j}}(Q) \leq 3^{n},
$$

which implies $f_{0} \in \mathcal{H}_{q^{\prime}}^{\varphi}\left(\mathbb{R}^{n}\right)$. 
We will verify that

$$
\lim _{j \rightarrow \infty} \int_{Q_{0}} f_{k_{j}}(x) d x=\int_{Q_{0}} f_{0}(x) d x
$$

for all $Q_{0} \in \mathcal{D}$. Once (53) is established, we will see that $f=f_{0}$ and hence $f \in \mathcal{H}_{q^{\prime}}^{\varphi}\left(\mathbb{R}^{n}\right)$ by virtue of the Lebesgue differentiation theorem because at least we know that $f_{0}$ locally in $L^{q^{\prime}}\left(\mathbb{R}^{n}\right)$. By the definition of $f_{k_{j}}$, we have

$$
\int_{Q_{0}} f_{k_{j}}(x) d x=\sum_{l=-\infty}^{\infty} \sum_{\substack{Q \in \mathcal{D}_{l} \\ Q_{0} \cap Q \neq \emptyset}} \lambda_{k_{j}}(Q) \int_{Q_{0}} b_{k_{j}}(Q)(x) d x .
$$

Note that

$$
\left\|b_{k_{j}}(Q)\right\|_{1} \leq\left|Q_{0} \cap Q\right|^{\frac{1}{q}}\left\|b_{k_{j}}(Q)\right\|_{q^{\prime}} \leq \frac{\varphi(\ell(Q))\left|Q \cap Q_{0}\right|^{\frac{1}{q}}}{|Q|^{\frac{1}{q}}} \leq \frac{\varphi(\ell(Q))}{|Q|^{\frac{1}{q}}}
$$

for any cube $Q$ containing $Q_{0}$.

We distinguish two cases. If

$$
\lim _{t \rightarrow \infty} \varphi(t) t^{-\frac{n}{q}}=0,
$$

then for all $\varepsilon>0$ there exists $l \in \mathbb{N}$ such that

$$
\sum_{l=N}^{\infty} \sum_{\substack{Q \in \mathcal{D}_{l} \\ Q 0 \cap Q \neq \emptyset}}\left|\lambda_{k_{j}}(Q) \int_{Q_{0}} b_{k_{j}}(Q)(x) d x\right|<\varepsilon
$$

Thus, we are in the position of using the Lebesgue convergence theorem based on Example 4.12. Thus we obtain (53). If

$$
\lim _{t \rightarrow \infty} \varphi(t) t^{-\frac{n}{q}}>0
$$

then we go through a similar argument using Example 4.28 to obtain (53).

Since $f_{k} \uparrow f$ a.e., we must have by (53)

$$
\int_{Q_{0}} f(x) d x=\int_{Q_{0}} f_{0}(x) d x
$$

for all $Q_{0} \in \mathcal{D}$. This yields $f=f_{0}$ a.e., by the Lebesgue differentiation theorem, and, hence, $f \in \mathcal{H}_{q^{\prime}}^{\varphi}\left(\mathbb{R}^{n}\right)$. Since we have verified $f \in \mathcal{H}_{q^{\prime}}^{\varphi}\left(\mathbb{R}^{n}\right)$, it follows that

$$
\|f\|_{\mathcal{H}_{q^{\prime}}^{\varphi}}=\sup \left\{\left|\int_{\mathbb{R}^{n}} f_{k}(x) g(x) d x\right|: k=1,2, \ldots,\|g\|_{\mathcal{M}_{q}^{\varphi}} \leq 1\right\} \leq 1 .
$$

This completes the proof of the theorem.

The proof of the following theorem is completely the same as the classical case once Proposition 4.30 is proved.

Theorem 4.31. Let $1<q<\infty$, and let $\varphi \in \mathcal{G}_{q}$ satisfy $\frac{1}{\varphi} \in \mathbb{Z}_{0}$. 
- The dual of $\mathcal{H}_{q^{\prime}}^{\varphi}\left(\mathbb{R}^{n}\right)$ is $\mathcal{M}_{q}^{\varphi}\left(\mathbb{R}^{n}\right)$. More precisely, we have the following mappings:

- Any $f \in \mathcal{M}_{q}^{\varphi}\left(\mathbb{R}^{n}\right)$ defines a continuous functional $L_{f}$ by:

$$
L_{f}: g \mapsto \int_{\mathbb{R}^{n}} f(x) g(x) d x \in \mathbb{C}
$$

on $\mathcal{H}_{q^{\prime}}^{\varphi}\left(\mathbb{R}^{n}\right)$.

- Conversely, every continuous functional $L$ on $\mathcal{H}_{q^{\prime}}^{\varphi}\left(\mathbb{R}^{n}\right)$ can be realized with $f \in \mathcal{M}_{q}^{\varphi}\left(\mathbb{R}^{n}\right)$ as $L=L_{f}$.

- The correspondence $f \in \mathcal{M}_{q}^{\varphi}\left(\mathbb{R}^{n}\right) \mapsto L_{f} \in\left(\mathcal{H}_{q^{\prime}}^{\varphi}\left(\mathbb{R}^{n}\right)\right)^{*}$ is an isomorphism. Furthermore

$$
\begin{aligned}
& \|f\|_{\mathcal{M}_{q}^{\varphi}}=\sup \left\{\left|\int_{\mathbb{R}^{n}} f(x) g(x) d x\right|: g \in \mathcal{H}_{q^{\prime}}^{\varphi}\left(\mathbb{R}^{n}\right),\|g\|_{\mathcal{H}_{q^{\prime}}^{\varphi}}=1\right\} \\
& \text { and } \\
& \|g\|_{\mathcal{H}_{q^{\prime}}^{\varphi}}=\sup \left\{\left|\int_{\mathbb{R}^{n}} f(x) g(x) d x\right|: f \in \mathcal{M}_{q}^{\varphi}\left(\mathbb{R}^{n}\right),\|f\|_{\mathcal{M}_{q}^{\varphi}}=1\right\} .
\end{aligned}
$$

- The dual of $\widetilde{\mathcal{M}}_{q}^{\varphi}\left(\mathbb{R}^{n}\right)$ is $\mathcal{H}_{q^{\prime}}^{\varphi}\left(\mathbb{R}^{n}\right)$ in the following sense.

- Any $f \in \mathcal{H}_{q^{\prime}}^{\varphi}\left(\mathbb{R}^{n}\right)$ defines a continuous functional $L_{f}^{\prime}$ by:

$$
L_{f}: g \mapsto \int_{\mathbb{R}^{n}} f(x) g(x) d x \in \mathbb{C}
$$

on $\widetilde{\mathcal{M}}_{q}^{\varphi}\left(\mathbb{R}^{n}\right)$.

- Conversely, every continuous functional L on $\widetilde{\mathcal{M}}_{q}^{\varphi}\left(\mathbb{R}^{n}\right)$ can be realized with $f \in \mathcal{H}_{q^{\prime}}^{\varphi}\left(\mathbb{R}^{n}\right)$ as $L=L_{f}^{\prime}$.

- The correspondence $f \in \mathcal{H}_{q^{\prime}}^{\varphi}\left(\mathbb{R}^{n}\right) \mapsto L_{f}^{\prime} \in\left(\widetilde{\mathcal{M}}_{q}^{\varphi}\left(\mathbb{R}^{n}\right)\right)^{*}$ is an isomorphism. Furthermore

$$
\begin{aligned}
& \|f\|_{\widetilde{\mathcal{M}}_{q}^{\varphi}}=\sup \left\{\left|\int_{\mathbb{R}^{n}} f(x) g(x) d x\right|: g \in \mathcal{H}_{q^{\prime}}^{\varphi}\left(\mathbb{R}^{n}\right),\|g\|_{\mathcal{H}_{q^{\prime}}^{\varphi}}=1\right\} \\
& \quad \text { and } \\
& \|g\|_{\mathcal{H}_{q^{\prime}}^{\varphi}}=\sup \left\{\left|\int_{\mathbb{R}^{n}} f(x) g(x) d x\right|: f \in \widetilde{\mathcal{M}}_{q}^{\varphi}\left(\mathbb{R}^{n}\right),\|f\|_{\widetilde{\mathcal{M}}_{q}^{\varphi}}=1\right\} .
\end{aligned}
$$

Proof.

- (a) This is a consequence of Proposition 4.26.

(b) We let $Q_{j}=2^{j}[-1,1]^{n}$. For the sake of the simplicity we denote $L^{q^{\prime}}\left(Q_{j}\right)$ by the set of $L^{q^{\prime}}\left(\mathbb{R}^{n}\right)$-functions supported on $Q_{j}$. The functional $g \mapsto L(g)$ is well defined and bounded on $L^{q^{\prime}}\left(Q_{j}\right)$. Thus by the duality $L^{p^{\prime}}-L^{p}$ there exists $f_{j}$ such that

$$
L(g)=\int_{Q_{j}} f_{j}(x) g(x) d x
$$


for all $g \in L^{q}\left(Q_{j}\right)$. By the uniqueness of this theorem we can find an $L_{\text {loc }}^{q}\left(\mathbb{R}^{n}\right)$ function $f$ such that $\left.f\right|_{Q_{j}}=f_{j}$ a.e. for any $j$.

We will prove $f \in \mathcal{M}_{q}^{p}\left(\mathbb{R}^{n}\right)$. For this purpose, we take an arbitrary $Q$ and estimate;

$$
I \equiv \varphi(\ell(Q))\left(\frac{1}{|Q|} \int_{Q}|f(x)|^{q} d x\right)^{\frac{1}{q}} .
$$

For a fixed cube $Q$ and a fixed function $f$ we set

$$
g(x) \equiv \chi_{Q}(x) \operatorname{sgn}(f(x))|f(x)|^{q-1} \quad x \in \mathbb{R}^{n} .
$$

Then we can write

$$
I=\varphi(\ell(Q))\left(\frac{1}{|Q|} \int_{Q} f(x) g(x) d x\right)^{\frac{1}{q}}=\varphi(\ell(Q))\left(\frac{1}{|Q|} L(g)\right)^{\frac{1}{q}} .
$$

Notice that the function $\frac{|Q|^{\frac{1}{p}-\frac{1}{q}}}{\|g\|_{q^{\prime}}} g$ is a $\left(p^{\prime}, q^{\prime}\right)$-block. Hence, we have $|L(g)| \leq \varphi(\ell(Q))^{-1}\|L\|_{*}|Q|^{\frac{1}{q}}\|g\|_{q^{\prime}}$. As a result we have $I \leq\|L\|_{*}$. This is the desired result.

(c) Carefully reexamine the proof of $(a)$ and (b). The proof of $(c)$ is already included in them.

- The heart of the matters is to prove (b); (a) and (c) are obtained similarly to (1). Let $L^{\prime}: \widetilde{\mathcal{M}}_{q}^{\varphi}\left(\mathbb{R}^{n}\right) \rightarrow \mathbb{C}$ be a bounded linear mapping. Since $\varphi^{-1} \in$ $\mathbb{Z}_{0}$, we have $\varphi(t) \lesssim t^{1 / P}, 0<t \leq 1$ for some $P>1$. For such $P>0$, we have $L^{P}\left(\mathbb{R}^{n}\right) \cap L_{\mathrm{c}}^{0}\left(\mathbb{R}^{n}\right) \hookrightarrow \mathcal{M}_{q}^{\varphi}\left(\mathbb{R}^{n}\right)$. As a consequence, for each $k \in$ $\mathbb{N}$ the mapping $f \in L^{P}\left(\mathbb{R}^{n}\right) \mapsto L^{\prime}\left(\chi_{[-k, k]^{n}} f\right) \in \mathbb{C}$ is a bounded linear mapping that can be realized by some $f_{k} \in L^{P^{\prime}}\left(\mathbb{R}^{n}\right)$. Furthermore, $f_{k}=$ $f_{k+1} \chi_{[-k, k]^{n}}$ from the $L P\left(\mathbb{R}^{n}\right)-L^{P^{\prime}}\left(\mathbb{R}^{n}\right)$-duality. So, $f=\lim _{k \rightarrow \infty} f_{k}$ exists almost everywhere. Let $h \in L_{\mathbf{c}}^{\infty}\left(\mathbb{R}^{n}\right)$ with $\operatorname{supp}(h) \subset[-k, k]^{n}$ for some $k \in \mathbb{N}$. As a result

$$
\int_{\mathbb{R}^{n}}\left|h(x) f_{k}(x)\right| d x=L\left(h \overline{\operatorname{sgn}}\left(f_{k}\right)\right) \leq\|L\|_{\widetilde{\mathcal{M}}_{q}^{\varphi} \rightarrow \mathbb{C}}\|h\|_{\widetilde{\mathcal{M}}_{q}^{\varphi}} .
$$

Since $h$ is arbitrary, it follows that $\left\|f_{k}\right\|_{\mathcal{H}_{q^{\prime}}^{\varphi}} \leq\|L\|_{\widetilde{\mathcal{M}}_{q}^{\varphi} \rightarrow \mathbb{C}}$. Using $f_{k}=$ $f_{k+1} \chi_{[-k, k]^{n}}$ and Proposition 4.30, we see that $f \in \mathcal{H}_{q^{\prime}}^{\varphi}\left(\mathbb{R}^{n}\right)$ and that $L_{f}^{\prime}\left|L_{\mathrm{c}}^{\infty}\left(\mathbb{R}^{n}\right)=L\right| L_{\mathrm{c}}^{\infty}\left(\mathbb{R}^{n}\right)$. Since $L_{\mathrm{c}}^{\infty}\left(\mathbb{R}^{n}\right)$ is dense in $\widetilde{\mathcal{M}}_{q}^{\varphi}\left(\mathbb{R}^{n}\right)$, it follows that $L=L_{f}^{\prime}$, as required.

We have the following conclusion.

Theorem 4.32. [73, Theorem 2] Let $1<q<\infty$ and let $\varphi \in \mathcal{G}_{q}$ satisfy $\frac{1}{\varphi} \in$ $\mathbb{Z}_{0}$. Then any singular integral operator, which is initially defined for $L_{\mathrm{c}}^{\infty}\left(\mathbb{R}^{n}\right)$ functions, can be naturally extended to a bounded linear operator on $\mathcal{M}_{q}^{\varphi}\left(\mathbb{R}^{n}\right)$. More precisely, $T$ has the following properties: 
- For all $f \in L_{\mathrm{c}}^{\infty}\left(\mathbb{R}^{n}\right), T f \in \widetilde{\mathcal{M}}_{q}^{\varphi}\left(\mathbb{R}^{n}\right)$ and satisfies $\|T f\|_{\widetilde{\mathcal{M}}_{q}^{\varphi}} \lesssim\|f\|_{\widetilde{\mathcal{M}}_{q}^{\varphi}}$. In particular $T$ extends to a bounded linear operator on $\widetilde{\mathcal{M}}_{q}^{\varphi}\left(\mathbb{R}^{n}\right)$.

- The adjoint operator $T^{*}$ is bounded on $\mathcal{H}_{q^{\prime}}^{\varphi}\left(\mathbb{R}^{n}\right)$.

- The adjoint $T^{* *}$ of $T^{*}$ is a bounded linear operator on $\mathcal{M}_{q}^{\varphi}\left(\mathbb{R}^{n}\right)$.

Theorem 4.32 extends the result by Peetre in [84] from Morrey spaces to generalized Morrey spaces. The proof is based on [87].

Proof. It suffices to prove the first assertion, since the remaining assertions are general facts on functional analysis. Since $\varphi \in \mathcal{G}_{q}, L_{\mathrm{c}}^{\infty}\left(\mathbb{R}^{n}\right)$ is a subset of $\widetilde{\mathcal{M}}_{q}^{\varphi}\left(\mathbb{R}^{n}\right)$. Let $f \in L_{\mathrm{c}}^{\infty}\left(\mathbb{R}^{n}\right)$. Then there exists $R>0$ such that $f$ is supported on $Q(R)$. We can decompose

$$
|T f| \lesssim \chi_{Q(2 R)}|T f|+(R+|\cdot|)^{-n} \int_{Q(R)}|f(y)| d y \in \widetilde{\mathcal{M}}_{q}^{\varphi}\left(\mathbb{R}^{n}\right),
$$

since

and hence

$$
(R+|\cdot|)^{-n \eta} \lesssim\left(M \chi_{Q(R)}\right)^{\eta} \in \mathcal{M}_{q}^{\varphi}\left(\mathbb{R}^{n}\right) \quad\left(q^{-1}<\eta<1\right)
$$

$$
(R+|\cdot|)^{-n}=\lim _{L \rightarrow \infty} \chi_{Q(L)}(R+|\cdot|)^{-n}
$$

in $\mathcal{M}_{q}^{\varphi}\left(\mathbb{R}^{n}\right)$. It thus remains to show that $\|T f\|_{\widetilde{\mathcal{M}}_{q}^{\varphi}} \lesssim\|f\|_{\widetilde{\mathcal{M}}_{q}^{\varphi}}$ for all $f \in L_{\mathrm{c}}^{\infty}\left(\mathbb{R}^{n}\right)$. We argue as follows: Let $Q$ be a cube. We need to show

$$
\varphi(\ell(Q))\left(\frac{1}{|Q|} \int_{Q}|T f(y)|^{q} d y\right)^{\frac{1}{q}} \lesssim\|f\|_{\mathcal{M}_{q}^{\varphi}} .
$$

We decompose this estimate according to $3 Q$ : Since $T$ is known to be $L^{q}\left(\mathbb{R}^{n}\right)$ bounded,

$$
\begin{aligned}
\varphi(\ell(Q))\left(\frac{1}{|Q|} \int_{Q}\left|T\left[\chi_{3 Q} f\right](y)\right|^{q} d y\right)^{\frac{1}{q}} & \leq \varphi(\ell(Q))\left(\frac{1}{|Q|} \int_{\mathbb{R}^{n}}\left|T\left[\chi_{3 Q} f\right](y)\right|^{q} d y\right)^{\frac{1}{q}} \\
& \lesssim \varphi(\ell(Q))\left(\frac{1}{|Q|} \int_{3 Q}|f(y)|^{q} d y\right)^{\frac{1}{q}} \\
& \leq \varphi(3 \ell(Q))\left(\frac{1}{|Q|} \int_{3 Q}|f(y)|^{q} d y\right)^{\frac{1}{q}} \\
& \lesssim\|f\|_{\mathcal{M}_{q}^{\varphi} .}
\end{aligned}
$$

Meanwhile, using the size condition of $T$, we have

$$
\begin{aligned}
\varphi(\ell(Q))\left(\frac{1}{|Q|} \int_{Q}\left|T\left[\chi_{\mathbb{R}^{n} \backslash 3 Q} f\right](y)\right|^{q} d y\right)^{\frac{1}{q}} & \leq \varphi(\ell(Q)) \sup _{x \in Q}\left|T\left[\chi_{\mathbb{R}^{n} \backslash 3 Q} f\right](x)\right| \\
& \lesssim \varphi(\ell(Q)) \int_{\mathbb{R}^{n} \backslash 3 Q} \frac{|f(y)|}{|y-c(Q)|^{n}} d y \\
& \lesssim \varphi(\ell(Q)) \sum_{m=1}^{\infty} \frac{1}{\left|2^{m} Q\right|} \int_{2^{m+1} Q \backslash 2^{m} Q}|f(y)| d y
\end{aligned}
$$


Since $\frac{1}{\varphi} \in \mathbb{Z}_{0}$, we obtain

$$
\begin{aligned}
& \varphi(\ell(Q))\left(\frac{1}{|Q|} \int_{Q}\left|T\left[\chi_{\mathbb{R}^{n} \backslash 3 Q} f\right](y)\right|^{q} d y\right)^{\frac{1}{q}} \\
& \lesssim \varphi(\ell(Q)) \sum_{m=1}^{\infty} \frac{1}{\varphi\left(2^{m} \ell(Q)\right)} \frac{\varphi\left(2^{m} \ell(Q)\right)}{\left|2^{m} Q\right|} \int_{2^{m+1} Q \backslash 2^{m} Q}|f(y)| d y \\
& \lesssim \varphi(\ell(Q)) \sum_{m=1}^{\infty} \frac{1}{\varphi\left(2^{m} \ell(Q)\right)}\|f\|_{\mathcal{M}_{q}^{\varphi}} \\
& \lesssim \varphi(\ell(Q))\|f\|_{\mathcal{M}_{q}^{\varphi}} \int_{\ell(Q)}^{\infty} \frac{1}{\varphi(s) s} d s \\
& \lesssim\|f\|_{\mathcal{M}_{q}^{\varphi} .}
\end{aligned}
$$

Thus we have $\|T f\|_{\widetilde{\mathcal{M}}_{q}^{\varphi}} \lesssim\|f\|_{\widetilde{\mathcal{M}}_{q}^{\varphi}}$ for all $f \in L_{\mathrm{c}}^{\infty}\left(\mathbb{R}^{n}\right)$.

We did not use (33) for the proof of boundedness of the Hardy-Littlewood maximal operator. However for the proof of boundedness of the singular integral operators, (33) is absolutely necessary as the following proposition shows:

Proposition 4.33. Let $1<q<\infty$ and $\varphi \in \mathcal{G}_{q}$. If $\left\|R_{1} f\right\|_{\mathrm{w} \mathcal{M}_{q}^{\varphi}} \lesssim\|f\|_{\mathcal{M}_{q}^{\varphi}}$ for all $f \in L_{\mathrm{c}}^{\infty}\left(\mathbb{R}^{n}\right)$, then $\frac{1}{\varphi} \in \mathbb{Z}_{0}$, where $R_{1}$ denotes the first Riesz transform.

Proof. Let $V \equiv\left\{x=\left(x_{1}, x_{2}, \ldots, x_{n}\right) \in \mathbb{R}^{n}: 2 x_{1}>|x|\right\}$. Assume that $\frac{1}{\varphi} \notin \mathbb{Z}_{0}$, so that for any $m \in \mathbb{N} \cap[3, \infty)$ there exists $r_{m}>0$ such that $\varphi\left(2^{m} r_{m}\right) \leq 2 \varphi\left(r_{m}\right)$. Then, consider $f_{m}=\chi_{V \cap B\left(2^{m-1} r_{m}\right) \backslash B\left(2 r_{m}\right)}$. Let $x \in V \cap B\left(r_{m}\right)$. If $y \in V \cap$ $B\left(2^{m-1} r_{m}\right) \backslash B\left(2 r_{m}\right)$, then $x-y \in V$ and $r_{m} \leq|x-y| \leq 2^{m} r_{m}$. Thus,

$$
\begin{aligned}
R_{1} f_{m}(x) & =\int_{\mathbb{R}^{n}} \frac{x_{1}-y_{1}}{|x-y|^{n+1}} f_{m}(y) d y \\
& =\int_{V \cap B\left(2^{m-1} r_{m}\right) \backslash B\left(2 r_{m}\right)} \frac{y_{1}}{|y|^{n+1}} d y \\
& \geq C \int_{V \cap B\left(2^{m-1} r_{m}\right) \backslash B\left(2 r_{m}\right)} \frac{1}{|y|^{n}} d y \\
& \simeq \log m .
\end{aligned}
$$

Since $V$ is a cone, we have

$$
\chi_{B\left(r_{m}\right)} \lesssim M \chi_{V \cap B\left(r_{m}\right)}
$$

We use this estimate and the boundedness of $M$ on $\mathcal{M}_{q}^{\varphi}\left(\mathbb{R}^{n}\right)$ to obtain

$$
\varphi\left(r_{m}\right) \lesssim\left\|\chi_{B\left(r_{m}\right)}\right\|_{\mathcal{M}_{q}^{\varphi}} \lesssim\left\|M \chi_{V \cap B\left(r_{m}\right)}\right\|_{\mathcal{M}_{q}^{\varphi}} \lesssim\left\|\chi_{V \cap B\left(r_{m}\right)}\right\|_{\mathcal{M}_{q}^{\varphi}}
$$


By using the inequality $\log m \lesssim\left|R_{1} f_{m}(x)\right|$ for $x \in V \cap B\left(r_{m}\right)$ and the boundedness of $R_{1}$ on $\mathcal{M}_{q}^{\varphi}\left(\ell^{u}\right)$, we have

$$
\begin{aligned}
\log (m) \varphi\left(r_{m}\right) & \lesssim\left\|R_{1} f_{m}\right\|_{\mathrm{w} \mathcal{M}_{q}^{\varphi}} \\
& \lesssim\left\|f_{m}\right\|_{\mathcal{M}_{q}^{\varphi}} \\
& \leq\left\|\chi_{B\left(2^{m} r_{m}\right)}\right\|_{\mathcal{M}_{q}^{\varphi}} \\
& \lesssim \varphi\left(2^{m} r_{m}\right) \\
& \sim \varphi\left(r_{m}\right) .
\end{aligned}
$$

This implies $\log m \leq D$ where $D$ is independent of $m$, contradictory to the fact that $m \geq 3$ is arbitrary. Hence, there exists some $m_{0} \in \mathbb{N}$ such that $\varphi\left(2^{m_{0}} r\right)>2 \varphi(r)$. Thus the integral condition (33) holds. $m_{q}^{p}\left(\mathbb{R}^{n}\right)$.

We disprove that $T$ can not be exteded to a bounded linear operator on

Example 4.34. Let $1 \leq q \leq p<\infty$. Then

$$
\left\|R_{1} f\right\|_{\mathrm{w}_{m_{q}^{p}}} \lesssim\|f\|_{m_{q}^{p}} \quad\left(f \in L_{\mathrm{c}}^{\infty}\left(\mathbb{R}^{n}\right)\right)
$$

and

$$
\left\|R_{1} f\right\|_{\mathrm{w} L_{\mathrm{uloc}}^{q}} \lesssim\|f\|_{L_{\mathrm{uloc}}^{q}} \quad\left(f \in L_{\mathrm{c}}^{\infty}\left(\mathbb{R}^{n}\right)\right)
$$

fail. In fact, let $\varphi(t)=\min \left(t^{\frac{n}{p}}, 1\right), t>0$ as in Example 3.4. Then $\varphi$ fails (33) because $\int_{1}^{\infty} \frac{d r}{\varphi(r)}=\infty$.

We end this section with extension to the vector-valued inequality.

Theorem 4.35. Let $1<q<\infty, 1<u<\infty$ and $\varphi \in \mathcal{G}_{q}$. Let also $T$ be a singular integral operartor. Assume in addition that (33) holds. Then for all $\left\{f_{j}\right\}_{j=1}^{\infty} \subset$ $\mathcal{M}_{q}^{\varphi}\left(\mathbb{R}^{n}\right)$,

$$
\left\|\left(\sum_{j=1}^{\infty}\left|T f_{j}\right|^{u}\right)^{\frac{1}{u}}\right\|_{\mathcal{M}_{q}^{\varphi}} \lesssim\left\|\left(\sum_{j=1}^{\infty}\left|f_{j}\right|^{u}\right)^{\frac{1}{u}}\right\|_{\mathcal{M}_{q}^{\varphi}}
$$

for all $\left\{f_{j}\right\}_{j=1}^{\infty} \subset \mathcal{M}_{q}^{\varphi}\left(\mathbb{R}^{n}\right)$.

Proof. Similar to Theorem 4.5. We also use a well-known inequality:

$$
\left\|\left(\sum_{j=1}^{\infty}\left|T f_{j}\right|^{u}\right)^{\frac{1}{u}}\right\|_{L^{q}} \lesssim\left\|\left(\sum_{j=1}^{\infty}\left|f_{j}\right|^{u}\right)^{\frac{1}{u}}\right\|_{L^{q}}
$$

for all $\left\{f_{j}\right\}_{j=1}^{\infty} \subset L^{q}\left(\mathbb{R}^{n}\right)$. 
Remark 4.36. One can consider the singular integral operators having rough kernel. Let $\Omega: S^{n-1} \equiv\left\{x \in \mathbb{R}^{n}:|x|=1\right\} \rightarrow \mathbb{C}$ be a measurable function having enough integrability and having integral zero. Then define $T_{\Omega}$ by

$$
T_{\Omega} f(x)=\int_{\mathbb{R}^{n}} \frac{\Omega(x-y)}{|x-y|^{n}} f(y) d y .
$$

See [5] for example.

Remark 4.37. N. Samko introduced a method of defining singular integral operators on generalized Morrey spaces by considering p-admissible singular integral operators in [88, Definition 3.3]. See also [31, Theorem 4.5] and [2, Theorem 4.3]. See [57] for a similar approach in the weighted setting.

Remark 4.38. See [84], where Peetre proved the boundedness of the singular integral operators on generalized Morrey-Campanato spaces.

Remark 4.39. See [29, Theorem 6.2] for the boundedness of the singular integral operators.

Remark 4.40. See [33, Theorems 5.3, 5.6 and 6.3] and [31, Theorem 5.6] for the boundedness of the commutators generated by singular integral operators and BMO.

Remark 4.41. Chen and Ding dealt with the parabolic singular integral operators in $[8]$.

Remark 4.42. Pang, Li and Wang dealt with the oscillatory integral operators in [83].

Remark 4.43. See [108, Theorems 2.3, 2.4 and 3.1], [120, Theorem 1.7] and [127, Theorem 3.1] for the boundedness of the singular integral operator on generalized Morrey spaces in the multilinear setting.

\section{Remark 4.44.}

- Wang discussed the boundedness of the intrinsic square functions in [122], where Wang did not have to resort to the duality argument. See also [31, Corollary 6.7], [23, Theorems 5 and 6], [57, Section 5.2] and [44, Theorem 1.4] for similar approaches. See $[9,124]$ for the case of the commutators.

- Karaman, Guliyev and Serbetci handled the pseudo-differential operators in [57, Section 5.1].

- Karaman, Guliyev and Serbetci handled the Marcinkiewicz operators in [57, Section 5.3].

- Karaman, Guliyev and Serbetci handled the Bochner-Riesz operators in [57, Section 5.4].

Remark 4.45. Let $T$ be a singular integral operator. In [60, Theorem 3.2] Komori and Mizuhara considered the operator of the form $(f, g) \in \mathcal{H}_{q^{\prime}}^{\varphi}\left(\mathbb{R}^{n}\right) \times \mathcal{M}_{q}^{\varphi}\left(\mathbb{R}^{n}\right) \mapsto$ $f \cdot T g-T f \cdot g \in H^{1}\left(\mathbb{R}^{n}\right)$ and obtained a factorization theorem as an application of the $\mathcal{M}_{q^{\prime}}^{\varphi}\left(\mathbb{R}^{n}\right)$-boundednes of the commutators. 
4.3. Generalized fractional integral operators in generalized Morrey spaces. To consider the operator like $(1-\Delta)^{-1}$, we are oriented to considering

$$
I_{\rho} f(x)=\int_{\mathbb{R}^{n}} f(y) \frac{\rho(|x-y|)}{|x-y|^{n}} d y
$$

for any suitable function $f$ on $\mathbb{R}^{n}$, where $\rho:(0, \infty) \rightarrow(0, \infty)$ is a suitable measurable function. Generalized Morrey spaces allow us to consider more general fractional integral operators.

Let us discuss what condition we need in order to guarantee that $I_{\rho}$ enjoys some boundedness property. As we did in [18, p. 761], we always assume that $\rho$ satisfies the "Dini condition" for $I_{\rho}$.

$$
\int_{0}^{1} \frac{\rho(s)}{s} d s<\infty
$$

so that $I_{\rho} \chi_{Q}(x)$ is finite for any cube $Q$ and $x \in \mathbb{R}^{n}$.

In addition, we also assume that $\rho$ satisfies the "growth condition": there exist constants $C>0$ and $0<2 k_{1}<k_{2}<\infty$ such that

$$
\sup _{\frac{r}{2}<s \leq r} \rho(s) \lesssim \int_{k_{1} r}^{k_{2} r} \frac{\rho(s)}{s} d s, \quad r>0,
$$

as was proposed by Perez [85]. Condition (64) is weaker than the usual doubling condition: there exists a constant $D>0$ such that

$$
\frac{1}{D} \leq \frac{\rho(r)}{\rho(s)} \leq D
$$

whenever $r>0$ and $s>0$ satisfy $r \leq 2 s \leq 4 r$.

Proposition 4.46. If $\rho:(0, \infty) \rightarrow(0, \infty)$ satisfies the doubling condition (65), then

$$
\sup _{\frac{r}{2} \leq s \leq r} \rho(s) \leq 2 D^{2} \int_{\frac{r}{2}}^{r} \rho(s) \frac{d s}{s} \text {. }
$$

Proof. Keeping in mind $\int_{\frac{1}{2} r}^{r} \frac{d s}{s}=\log 2<1$, we calculate

$$
\sup _{\frac{r}{2} \leq s \leq r} \rho(s) \leq D \rho(r)=\frac{D}{\log 2} \int_{\frac{r}{2}}^{r} \rho(r) \frac{d s}{s} \leq \frac{D^{2}}{\log 2} \int_{\frac{r}{2}}^{r} \rho(s) \frac{d s}{s} \leq 2 D^{2} \int_{\frac{r}{2}}^{r} \rho(s) \frac{d s}{s} .
$$

Example 4.47. Let $0<\alpha<\infty$.

- $\rho(t)=t^{\alpha}$, which generates $I_{\alpha}$, satisfies the doubling condition.

- $\rho(t)=\frac{t^{\alpha}}{\log (e+t)}$ satisfies the doubling condition.

- $\rho(t)=\frac{t^{\alpha}}{1+t^{M}}$ satisfies the doubling condition. See [61] for an application to Schrödinger equations.

- $\rho(t)=t^{\alpha} e^{-t}$ satisfies the growth condition but fails the doubling condition. 
- Let $0 \leq \gamma<\infty$ and $\beta_{1}, \beta_{2} \in \mathbb{R}$. We set

$$
\ell^{\mathbb{B}}(r) \equiv \begin{cases}(1+|\log r|)^{\beta_{1}} & (0<r \leq 1) \\ (1+|\log r|)^{\beta_{2}} & (1<r<\infty) .\end{cases}
$$

as before. Then $\rho(t)=t^{\gamma} \ell^{\mathbb{B}}(t)$ satisfies (63) if and only if $\gamma=0>-1>\beta_{1}$ or $\gamma>0$. Meanwhile (65) is always satisfied. Noteworthy is the fact that we can tolerate the case $\gamma=0$ if $\beta_{1}<-1$.

To check that our example is not so artificial we consider the following kernel.

Definition 4.48. One defines $(1-\Delta)^{-\frac{s}{2}} f$ by

$$
(1-\Delta)^{-\frac{s}{2}} f=G_{s} * f
$$

where $G_{s}$ is given by:

$$
G_{s}(x)=\lim _{\varepsilon \downarrow 0} \frac{1}{(2 \pi)^{n}} \int_{\mathbb{R}^{n}} \frac{\exp \left(-|\varepsilon \xi|^{2}\right) e^{i x \cdot \xi}}{\left(1+|\xi|^{2}\right)^{\frac{s}{2}}} d \xi \quad\left(x \in \mathbb{R}^{n}\right) .
$$

The function $G_{s}$ is called the Bessel kernel.

The above examples are natural in some sense but somewhat artificial because the second example and the third one do not appear naturally in the context of other areas of mathematics. Here we present some other examples related to partial differential equations.

Example 4.49. Note that the solution to $(1-\Delta) f=g$, where $f$ is an unknown function and $g$ is a give function is given by:

$$
g=(1-\Delta)^{-1} f
$$

Although it is impossible to find $I_{\rho} \chi_{B(x, r)}(y), y \in \mathbb{R}^{n}$, we still have a partial but important estimate.

Lemma 4.50. Let $\rho:(0, \infty) \rightarrow(0, \infty)$ be a measurable function. Then inequality $\tilde{\rho}(R / 2) \lesssim I_{\rho} \chi_{B(R)}(x)$ holds whenever $x \in B(R / 2)$ and $R>0$.

Proof. Take $x \in B(R / 2)$. We write the integral in full:

$$
I_{\rho} \chi_{B(R)}(x)=\int_{\mathbb{R}^{n}} \frac{\rho(|x-y|)}{|x-y|^{n}} \chi_{B(R)}(y) d y=\int_{B(R)} \frac{\rho(|x-y|)}{|x-y|^{n}} d y .
$$

A geometric observation shows that $B(x, R / 2) \subseteq B(R)$. Hence, we have

$$
I_{\rho} \chi_{B(R)}(x) \geq \int_{B(x, R / 2)} \frac{\rho(|x-y|)}{|x-y|^{n}} d y=C \int_{0}^{R / 2} \frac{\rho(s)}{s} d s .
$$

Note that we only use the spherical coordinates to obtain the last integral. $x$ small.

In the case of the radially symmetric functions, we can calculate $I_{\rho} g_{R}(x)$ for 
Lemma 4.51. [18, Lemma 2.2] For every $R>0$ and a measurable function $\theta$ : $(0, \infty) \rightarrow[0, \infty)$ satisfying the doubling condition

$$
\theta(s) \sim \theta(r) \quad(0<r \leq s \leq 2 r)
$$

the inequality

$$
\int_{2 R}^{\infty} \frac{\theta(t) \rho(t)}{t} d t \lesssim I_{\rho} g_{R}(x) \lesssim \int_{2 R / 3}^{\infty} \frac{\theta(t) \rho(t)}{t} d t
$$

holds whenever $x \in B\left(\frac{R}{3}\right)$, where $g_{R}(x) \equiv \theta(|x|) \chi_{B(R)^{\mathrm{c}}}(x)$.

Proof. We prove the right-hand inequality, the left-hand inequality being similar. A geometric observation shows that $|x-y| \sim|y|$ for all $x \in B\left(\frac{R}{3}\right)$ and $y \in$ $\mathbb{R}^{n} \backslash B\left(\frac{2 R}{3}\right)$. Since $\theta$ satisfies $(66)$, then

$$
\begin{aligned}
I_{\rho} g_{R}(x) & =\int_{\mathbb{R}^{n} \backslash B(R)} \frac{\theta(|y|) \rho(|x-y|)}{|x-y|^{n}} d y \\
& \leq \int_{\mathbb{R}^{n} \backslash B(x, 2 R / 3)} \theta(|y|) \frac{\rho(|x-y|)}{|x-y|^{n}} d y \\
& =\int_{\mathbb{R}^{n} \backslash B\left(\frac{2 R}{3}\right)} \theta(|x-y|) \frac{\rho(|y|)}{|y|^{n}} d y \\
& \lesssim \int_{2 R / 3}^{\infty} \frac{\theta(t) \rho(t)}{t} d t \text { for } x \in B\left(\frac{R}{3}\right) .
\end{aligned}
$$

It remains to write the most right-hand side in terms of the spherical coordinates.

For covenience, write

$$
\tilde{\rho}(r) \equiv \int_{0}^{r} \frac{\rho(t)}{t} d t .
$$

Sometimes, we are interested in the case where matters are reduced to the classical fractional integral operators.

Proposition 4.52. Let $\rho:(0, \infty) \rightarrow(0, \infty)$ be a measurable function satisfying (64). Then the following are equivalent:

(a) $\rho(r) \lesssim r^{\alpha}$ for all $r>0$.

(b) $\tilde{\rho}(r) \lesssim r^{\alpha}$ for all $r>0$.

Proof. Clearly $(a)$ implies $(b)$, since $\int_{0}^{r} s^{\alpha-1} d s=\frac{1}{\alpha} r^{\alpha}$. Let us see $(b)$ implies $(a)$. Combining (64) with (b), we obtain

$$
\rho(r) \leq \sup _{\frac{r}{2}<s \leq r} \rho(s) \lesssim \int_{k_{1} r}^{k_{2} r} \frac{\rho(s)}{s} d s \lesssim \int_{0}^{k_{2} r} \frac{\rho(s)}{s} d s \lesssim\left(k_{2} r\right)^{\alpha} \sim r^{\alpha}
$$


Now we present three different criteria for the boundedness of $I_{\rho}$. We prove the following three theorems on the boundedness of $I_{\rho}$ on generalized Morrey spaces.

For the case of $q=1$, we have the following simple result:

Theorem 4.53. [18, Theorem 1.3] Let $1 \leq p<\infty$, and let $\varphi \in \mathcal{G}_{p}$ and $\psi \in \mathcal{G}_{1}$. Let also $\rho:(0, \infty) \rightarrow(0, \infty)$ be a measurable function satisfying $(64)$. Then $I_{\rho}$ is bounded from $\mathcal{M}_{p}^{\varphi}\left(\mathbb{R}^{n}\right)$ to $\mathcal{M}_{1}^{\psi}\left(\mathbb{R}^{n}\right)$ only if

$$
\frac{1}{\varphi(r)} \int_{0}^{r} \frac{\rho(t)}{t} d t+\int_{r}^{\infty} \frac{\rho(t)}{t \varphi(t)} d t \lesssim \frac{1}{\psi(r)} \quad(r>0) .
$$

Furthermore, if (68) is satisfied, then $I_{\rho}$ is bounded from $\mathcal{M}_{1}^{\varphi}\left(\mathbb{R}^{n}\right)$ to $\mathcal{M}_{1}^{\psi}\left(\mathbb{R}^{n}\right)$.

Note that the left-hand side of (68) equals

$$
\frac{1}{\varphi(r)} \int_{0}^{r} \frac{\rho(t)}{t} d t+\int_{r}^{\infty} \frac{\rho(t)}{t \varphi(t)} d t=\int_{0}^{\infty} \frac{\rho(t)}{t \varphi(\max (r, t))} d t .
$$

Proof of Theorem 4.53(Necessity). Assume that $I_{\rho}$ is a bounded linear operator from $\mathcal{M}_{p}^{\varphi}\left(\mathbb{R}^{n}\right)$ to $\mathcal{M}_{1}^{\psi}\left(\mathbb{R}^{n}\right)$. Let $r>0$. By Lemma 4.50 and the doubling property of $\psi$, we obtain

$$
\tilde{\rho}(r) \lesssim \frac{1}{r^{n}} \int_{B(r / 2)} I_{\rho} \chi_{B(r)}(x) d x \leq \frac{1}{r^{n}} \int_{B(r / 2)} I_{\rho} \chi_{B(r)}(x) d x \leq \frac{1}{\psi(r)}\left\|I_{\rho} \chi_{B(r)}\right\|_{\mathcal{M}_{1}^{\psi}}
$$

Since $\psi \in \mathcal{G}_{1}$ and $I_{\rho}$ is assumed bounded from $\mathcal{M}_{p}^{\varphi}\left(\mathbb{R}^{n}\right)$ to $\mathcal{M}_{1}^{\psi}\left(\mathbb{R}^{n}\right)$, it follows that

$$
\tilde{\rho}(r) \lesssim \frac{1}{\psi(r)}\left\|\chi_{B(r)}\right\|_{\mathcal{M}_{p}^{\varphi}}
$$

Since $\left\|\chi_{B(r)}\right\|_{\mathcal{M}_{p}^{\varphi}} \sim \varphi(r)$, we conclude

$$
\tilde{\rho}(r) \lesssim \frac{\varphi(r)}{\psi(r)}
$$

Let $g_{r}(x)=\frac{\chi_{B(r)^{c}(x)}}{\varphi(|x|)}$ for $x \in \mathbb{R}^{n}$. By Lemma 4.51 with $\theta=\frac{1}{\varphi}$, we have

$$
\int_{r}^{\infty} \frac{\rho(t)}{t \varphi(t)} d t \lesssim \psi\left(\frac{r}{6}\right)^{-1}\left\|I_{\rho} g_{r}\right\|_{\mathcal{M}_{1}^{\psi}} \lesssim \psi(r)^{-1}\left\|g_{r}\right\|_{\mathcal{M}_{1}^{\varphi}} \lesssim \psi(r)^{-1}
$$

Thus Theorem 4.53 is proved.

Proof of Theorem 4.53(Sufficiency). For a ball $B(z, r)$, we let $f_{1} \equiv f \chi_{B(z, 2 r)}$ and $f_{2} \equiv f-f_{1}$. Then a geometric observation shows $B(z, r) \subset B(y, 3 r)$ for all $y \in$ 
$B(z, 2 r)$. Hence by the Fubini theorem and the normalization,

$$
\begin{aligned}
\int_{B(z, r)}\left|I_{\rho} f_{1}(x)\right| d x & \leq \int_{B(z, r)}\left(\int_{B(z, 2 r)}|f(y)| \frac{\rho(|x-y|)}{|x-y|^{n}} d y\right) d x \\
& \leq \int_{B(z, 2 r)}\left(\int_{B(y, 3 r)}|f(y)| \frac{\rho(|x-y|)}{|x-y|^{n}} d x\right) d y \\
& =\int_{B(z, 2 r)}|f(y)| d y \times \int_{B(3 r)} \frac{\rho(|x|)}{|x|^{n}} d x
\end{aligned}
$$

By the use of the definition of the Morrey norm, (68) and the doubling condition of $\psi$, we obtain

$$
\begin{aligned}
\int_{B(z, r)}\left|I_{\rho} f_{1}(x)\right| d x & \lesssim \tilde{\rho}(3 r) \varphi(2 r)^{-1} r^{n} \\
& \lesssim \tilde{\rho}(3 r) \varphi(3 r)^{-1} r^{n} \\
& \lesssim \psi(3 r)^{-1} r^{n} \\
& \lesssim \psi(r)^{-1} r^{n}
\end{aligned}
$$

Thus the estimate for $f_{1}$ is valid. As for $f_{2}$, we let $x \in B(z, r)$. Then we have

$$
\left|I_{\rho} f_{2}(x)\right| \leq \int_{B(z, 2 r)^{\mathrm{c}}}|f(y)| \frac{\rho(|x-y|)}{|x-y|^{n}} d y \leq \int_{B(x, r)^{\mathrm{c}}}|f(y)| \frac{\rho(|x-y|)}{|x-y|^{n}} d y
$$

and decomposing the right-hand side dyadically we obtain

$$
\left|I_{\rho} f_{2}(x)\right| \leq \sum_{j=1}^{\infty} \int_{B\left(x, 2^{j} r\right) \backslash B\left(x, 2^{j-1} r\right)}|f(y)| \frac{\rho(|x-y|)}{|x-y|^{n}} d y \lesssim \int_{2 k_{1} r}^{\infty} \frac{\rho(t)}{t \varphi(t)} d t .
$$

If we use (68) once again and the doubling condition on $\psi$, then we obtain $\left|I_{\rho} f_{2}(x)\right| \lesssim$ $\psi(r)^{-1}$. Thus the estimate for $f_{2}$ is valid as well.

In the following example, we consider why we need generalized Morrey spaces.

Example 4.54. [105, Theorem 5.1], [18, Example 5.1] Let $s \in(0, n)$ and $\kappa>0$. Define $\psi(r) \equiv \frac{(1+r)^{s}}{\max \left(1, \log r^{-1}\right)} \quad(r>0)$. Let $\rho(r)=r^{s} \exp (-\kappa r), \varphi(r)=r^{s}$ for $r>0$. Then $\rho$ is a measurable function satisfying (64). Furthermore, if $0<$ $r<1, \frac{1}{\varphi(r)} \int_{0}^{r} \frac{\rho(t)}{t} d t+\int_{r}^{\infty} \frac{\rho(t)}{t \varphi(t)} d t \sim \frac{1}{\psi(r)}$ and if $r \geq 1, \frac{1}{\varphi(r)} \int_{0}^{r} \frac{\rho(t)}{t} d t+$ $\int_{r}^{\infty} \frac{\rho(t)}{t \varphi(t)} d t \lesssim \frac{1}{\psi(r)}$. Thus $\left\|(1-\Delta)^{-\frac{s}{2}} f\right\|_{\mathcal{M}_{1}^{\psi}} \lesssim_{s}\left\|I_{\rho} f\right\|_{\mathcal{M}_{1}^{\psi}} \lesssim\|f\|_{\mathcal{M}_{1}^{\frac{n}{s}}}$ for all $f \in$ $\mathcal{M}_{1}^{\frac{n}{s}}\left(\mathbb{R}^{n}\right)$. This calculation shows we cannot delete $\max \left(1, \log r^{-1}\right)$ and that $(1-$ $\Delta)^{-\frac{s}{2}}$ does not map $\mathcal{M}_{q}^{p}\left(\mathbb{R}^{n}\right)$ to $L^{\infty}\left(\mathbb{R}^{n}\right)$ when $\frac{n}{p}=s$ and $1<q \leq p<\infty$.

Example 4.54 convince us that generalized Morrey spaces occur naturally. 
Example 4.55. Let $\frac{1}{s}=\frac{1}{p}-\frac{\alpha}{n}$ with $1<p<s<\infty$ and $0<\alpha<n$. Then $I_{\alpha}$ does not map $m_{1}^{p}\left(\mathbb{R}^{n}\right)$ to $m_{1}^{s}\left(\mathbb{R}^{n}\right)$, since $\rho(t) \equiv t^{\alpha}, t \geq 0$ and $\varphi(t) \equiv \max \left(t^{\frac{n}{p}}, 1\right), t>0$ satisfies

instead of

$$
\int_{r}^{\infty} \frac{\rho(t)}{t \varphi(t)} d t=\infty
$$

$$
\int_{r}^{\infty} \frac{\rho(t)}{t \varphi(t)} d t \lesssim \frac{1}{\varphi(t)^{\frac{s}{p}}}
$$

If we consider the truncated fractional maximal operator $i_{\alpha}$ given by

$$
i_{\alpha} f(x)=\int_{|y| \leq 1} \frac{f(x-y)}{|y|^{n-\alpha}} d y
$$

then $i_{\alpha}$ maps $m_{1}^{p}\left(\mathbb{R}^{n}\right)$ to $m_{1}^{s}\left(\mathbb{R}^{n}\right)$, since

$$
\frac{1}{\varphi(r)} \int_{0}^{r} \frac{\rho(t) \chi_{(0,1)}(t)}{t} d t+\int_{r}^{\infty} \frac{\rho(t) \chi_{(0,1)}(t)}{t \varphi(t)} d t \lesssim \frac{1}{\varphi(t)^{\frac{s}{p}}} .
$$

Example 4.56. [105, Theorem 5.1], [18, Example 5.1] Let $0<s<n$. Define $\varphi(r) \equiv r^{s}$ and $\psi(r) \equiv(1+r)^{-s} \ell^{(-1,0)}(r)$ for $r>0$. Let $\rho(r) \equiv r^{n} G_{s}(r)$, where $G_{s}$ denotes the Bessel kernel, the kernel of $(1-\Delta)^{\frac{s}{2}}$. Observe that $\tilde{\rho}(r) \sim \min \left(r^{s}, 1\right)$ and hence $\frac{\tilde{\rho}(r)}{\varphi(r)} \sim \min \left(1, r^{-s}\right)$. Note also that

$$
\int_{r}^{\infty} \frac{\rho(t)}{t \varphi(t)} d t \sim \begin{cases}\log (e / r) & (r<1) \\ r^{n-s} G_{s}(r) & (r \geq 1)\end{cases}
$$

Then

$$
\frac{\tilde{\rho}(r)}{\varphi(r)}+\int_{r}^{\infty} \frac{\rho(t)}{t \varphi(t)} d t \sim \frac{1}{\psi(r)} \quad(r>0) .
$$

Hence it follows from Theorem 4.53 that $\left\|I_{\rho} f\right\|_{\mathcal{M}_{1}^{\psi}} \lesssim\|f\|_{\mathcal{M}_{1}^{\varphi}}$, extending Proposition 4.54. This triple $(\rho, \varphi, \psi)$ fulfills the assumption (68). However, $\frac{\rho}{\varphi} \notin \mathbb{Z}_{0}$ since $\frac{\rho(r)}{\varphi(r)}=\mathrm{o}(1)$ as $r \downarrow 0$ and (69) fails.

We give a result, which improves Example 4.54.

We move on to the Adams type estimate.

Theorem 4.57. [18, Theorems 1.1 and 1.2] Let $1<p<q<\infty$ and $\varphi \in \mathcal{G}_{p}$. Assume that $\rho:(0, \infty) \rightarrow(0, \infty)$ satisfies $(64)$.

- The operator $I_{\rho}$ is bounded from $\mathcal{M}_{p}^{\varphi}\left(\mathbb{R}^{n}\right)$ to $\mathcal{M}_{q}^{\varphi^{p / q}}\left(\mathbb{R}^{n}\right)$ if

$$
\frac{1}{\varphi(r)} \int_{0}^{r} \frac{\rho(t)}{t} d t+\int_{r}^{\infty} \frac{\rho(t)}{t \varphi(t)} d t \lesssim \frac{1}{\varphi(r)^{\frac{p}{q}}}
$$

for all $r>0$. If $\varphi \in \mathbb{Z}^{-\frac{n}{p}}$, then (69) is necessary for the boundedness of $I_{\rho}$ from $\mathcal{M}_{p}^{\varphi}\left(\mathbb{R}^{n}\right)$ to $\mathcal{M}_{q}^{\varphi^{p / q}}\left(\mathbb{R}^{n}\right)$. 
- Assume $\frac{\rho}{\varphi} \in \mathbb{Z}_{0}$. Then $I_{\rho}$ is bounded from $\mathcal{M}_{p}^{\varphi}\left(\mathbb{R}^{n}\right)$ to $\mathcal{M}_{q}^{\varphi^{p / q}}\left(\mathbb{R}^{n}\right)$ if and only if

$$
\tilde{\rho}(r) \lesssim \varphi(r)^{1-p / q} \quad(r>0) .
$$

So, if $\frac{\rho}{\varphi} \in \mathbb{Z}_{0}$, condition (69) simplies to (70).

\section{Remark 4.58.}

- The first half of "only if" part (70) is clear from Theorem 4.53 with $\psi=$ $\varphi^{p / q}$.

- Once we assume $\frac{\rho}{\varphi} \in \mathbb{Z}_{0}$, it is eacy to check that (70) implies (69). Indeed, if we use $\frac{\rho}{\varphi} \in \mathbb{Z}_{0}$ and $\varphi \in \mathcal{G}_{p}$, then we have

$$
\int_{r}^{\infty} \frac{\rho(s)}{s \varphi(s)} d s \lesssim \frac{\rho(r)}{\varphi(r)} .
$$

Since $\rho$ satisfies the growth condition, we have

$$
\int_{r}^{\infty} \frac{\rho(s)}{s \varphi(s)} d s \lesssim \frac{\tilde{\rho}\left(k_{2} r\right)}{\varphi(r)} .
$$

If we use (70) and the doubling condition on $\varphi$, then we obtain

$$
\int_{r}^{\infty} \frac{\rho(t)}{t \varphi(t)} d t \lesssim \frac{1}{\varphi(r)^{\frac{p}{q}}}
$$

- For the "if" part we only need the following estimate of Hedberg-type, see Lemma 4.59 below.

Proof of Theorem 4.57, necessity. According to Theorem 4.53, we have only to show

$$
\int_{2 R}^{\infty} \frac{\rho(t)}{t \varphi(t)} d t \lesssim \varphi(2 R)^{-p / q}
$$

By virtue of Lemma 4.51, we obtain

$$
\int_{2 R}^{\infty} \frac{\rho(t)}{t \varphi(t)} d t \sim\left(\frac{1}{R^{n}} \int_{B\left(\frac{R}{3}\right)} I_{\rho} g(x)^{q} d x\right)^{\frac{1}{q}} \lesssim \varphi(R)^{-p / q}\left\|I_{\rho} g_{R}\right\|_{\mathcal{M}_{q}^{\varphi^{p / q}}} .
$$

Since $I_{\rho}$ is bounded, we obtain

$$
\int_{2 R}^{\infty} \frac{\rho(t)}{t \varphi(t)} d t \lesssim \varphi(R)^{-p / q}\left\|g_{R}\right\|_{\mathcal{M}_{p}^{\varphi}} \lesssim \varphi(R)^{-p / q}\left\|\frac{1}{\varphi(|\cdot|)}\right\|_{\mathcal{M}_{p}^{\varphi}} .
$$

Recall that we are assuming $\varphi \in \mathbb{Z}^{-\frac{n}{p}}$. Now we invoke Lemma 4.17 to conclude

$$
\int_{2 R}^{\infty} \frac{\rho(t)}{t \varphi(t)} d t \lesssim \varphi(R)^{-p / q} \lesssim \varphi(2 R)^{-p / q} .
$$

Thus necessity is proven.

As we have mentioned, we want an estimate of Hedberg-type. We may ask ourselves whether $\inf _{r>0} \frac{1}{\varphi(r)^{\frac{p}{q}}}$ can be removed, that is, we may assume $\sup _{t>0} \varphi(t)=\infty$. However, it can happen that $\sup _{t>0} \varphi(t)<\infty$ as example below shows. 
Lemma 4.59. [18, Lemma 3.1] Let $1 \leq p<q<\infty$, and let $\varphi \in \mathcal{G}_{p} \cap W$ satisfy (69). Let $\rho:(0, \infty) \rightarrow(0, \infty)$ be a measurable function satisfying (64). If a measurable function $f$ satsfies $\|f\|_{\mathcal{M}_{p}^{\varphi}}=1$, then

$$
\left|I_{\rho} f(x)\right| \lesssim[M f(x)]^{p / q}+\inf _{r>0} \varphi(r)^{-\frac{p}{q}}
$$

for $x \in \mathbb{R}^{n}$.

Once this estimate is satisfied, we can conclude the proof of Theorem 4.57 as follows: We choose an arbitrary ball $B=B(z, r)$. If we integrate Lemma 4.59, then we have

$$
\frac{1}{|B|} \int_{B}\left|I_{\rho} f(x)\right|^{q} d x \lesssim \frac{1}{|B|} \int_{B}[M f(x)]^{p} d x+\inf _{u>0} \varphi(u)^{-p} .
$$

If we multiply both sides by $\varphi(r)^{p}$, then we have

$$
\frac{\varphi(r)^{p}}{|B|} \int_{B}\left|I_{\rho} f(x)\right|^{q} d x \lesssim\left(\frac{\varphi(r)^{p}}{|B|} \int_{B}[M f(x)]^{p} d x+1\right) \lesssim 1
$$

by virtue of the boundedness of the maximal operator $M$ on $\mathcal{M}_{p}^{\varphi}\left(\mathbb{R}^{n}\right)$. The ball $B$ being arbitrary, we obtain the desired result.

Proof. Recall that $k_{1}$ and $k_{2}$ appeared in the condition (64) on $\rho$. Let

$$
\rho^{*}(r) \equiv \int_{k_{1} r}^{k_{2} r} \frac{\rho(s)}{s} d s .
$$

We have

$$
\left|I_{\rho} f(x)\right| \lesssim \sum_{j=-\infty}^{-1}+\sum_{j=0}^{\infty} \frac{\rho^{*}\left(2^{j} r\right)}{\left(2^{j} r\right)^{n}} \int_{|x-y|<2^{j} r}|f(y)| d y
$$

for given $x \in \mathbb{R}^{n}$ and $r>0$. Let $\Sigma_{I}$ and $\Sigma_{I I}$ be the first and second summations above. Now we invoke the overlapping property:

$$
\sum_{j=-\infty}^{-1} \chi_{\left[2^{j} k_{1} r, 2^{j} k_{2} r\right]} \lesssim \chi_{\left(-\infty, 2^{-1} k_{2} r\right]}, \quad \sum_{j=0}^{\infty} \chi_{\left[2^{j} k_{1} r, 2^{j} k_{2} r\right]} \lesssim \chi_{\left[k_{1} r, \infty\right)} .
$$

As a result, we have

$$
\sum_{j=-\infty}^{-1} \rho^{*}\left(2^{j} r\right) \leq \sum_{j=-\infty}^{-1} \int_{2^{j} k_{1} r}^{2^{j} k_{2} r} \frac{\rho(s)}{s} d s \lesssim \int_{0}^{k_{2} r} \frac{\rho(s)}{s} d s=\tilde{\rho}\left(k_{2} r\right)
$$

and

$$
\sum_{j=0}^{\infty} \frac{\rho^{*}\left(2^{j} r\right)}{\varphi\left(2^{j} r\right)} \lesssim \int_{k_{1} r}^{\infty}\left(\sum_{j=0}^{\infty} \chi_{\left[2^{j} k_{1} r, 2^{j} k_{2} r\right]}(s)\right) \frac{\rho(s)}{s \varphi(s)} d s \lesssim \int_{k_{1} r}^{\infty} \frac{\rho(s)}{s \varphi(s)} d s .
$$

Thus thanks to $(70)$

$$
\Sigma_{I} \lesssim \sum_{j=-\infty}^{-1} \rho^{*}\left(2^{j} r\right) M f(x) \leq C \tilde{\rho}\left(k_{2} r\right) M f(x) \lesssim \varphi(r)^{1-p / q} M f(x) .
$$


Meanwhile

$$
\Sigma_{I I} \lesssim \sum_{j=0}^{\infty} \frac{\rho^{*}\left(2^{j} r\right)}{\varphi\left(2^{j} r\right)}\|f\|_{\mathcal{M}_{p}^{\varphi}} \lesssim \int_{k_{1} r}^{\infty} \frac{\rho(s)}{s \varphi(s)} d s .
$$

We use $\frac{\rho}{\varphi} \in \mathbb{Z}_{0}$ or (69) now. If we use (69), then we have

$$
\int_{r}^{\infty} \frac{\rho(t)}{t \varphi(t)} d t \lesssim \frac{1}{\varphi(r)^{\frac{p}{q}}}
$$

By the doubling property of $\varphi$, we obtain $\Sigma_{I I} \lesssim \frac{1}{\varphi(r)^{\frac{p}{q}}}$. Hence,

$$
\left|I_{\rho} f(x)\right| \lesssim, \varphi(r)^{1-p / q}\left(M f(x)+\frac{1}{\varphi(r)}\right)
$$

for all $r>0$

First assume $M f(x) \leq \inf _{r>0} \frac{1}{\varphi(r)}$. Then, the conclusion is immediate from (73).

Next, we assume $M f(x)>\inf _{r>0} \frac{1}{\varphi(r)}$. Since $\|f\|_{\mathcal{M}_{p}^{\varphi}}=1$, we have

$$
1 \geq \varphi(r)\left(\frac{1}{|Q(x, r)|} \int_{Q(x, r)}|f(y)|^{p} d y\right)^{\frac{1}{p}} \geq \frac{\varphi(r)}{|Q(x, r)|} \int_{Q(x, r)}|f(y)| d y .
$$

Hence

for all $r>0$. This implies

$$
\frac{1}{|B(x, r)|} \int_{B(x, r)}|f(y)| d y \leq \frac{1}{\varphi(r)}
$$

$$
\frac{1}{|B(x, r)|} \int_{B(x, r)}|f(y)| d y \leq \sup _{R>0} \frac{1}{\varphi(R)}
$$

for all $r>0$. Since $r>0$ and $x \in \mathbb{R}^{n}$ are arbitrary, it follows that $\operatorname{Mf}(x) \leq$ $\sup _{r>0} \frac{1}{\varphi(r)}$. We can thus find $R>0$ such that $M f(x)=2 \varphi(R)$ and, with this $R$, we can obtain the desired estimate.

In order that $I_{\rho}$ be bounded from $\mathcal{M}_{p}^{\varphi}\left(\mathbb{R}^{n}\right)$ to $\mathcal{M}_{q}^{\varphi^{p / q}}\left(\mathbb{R}^{n}\right)$, we must have

$$
\frac{\tilde{\rho}(r)}{\varphi(r)} \lesssim \frac{1}{\varphi(r)^{\frac{p}{q}}}
$$

according to Theorem 4.53 with $\psi=\varphi^{p / q}$.

We note that if $\rho(r)=r^{\alpha}$, with $0<\alpha<n$, then $I_{\rho}=I_{\alpha}$ is the classical fractional integral operator, also known as the Riesz potential, which is bounded from $L^{p}\left(\mathbb{R}^{n}\right)$ to $L^{q}\left(\mathbb{R}^{n}\right)$ if and only if $\frac{1}{p}-\frac{1}{q}=\frac{\alpha}{n}$, where $1<p, q<\infty$. The necessary part is usually proved by using the scaling arguments.

Theorem 4.57 characterizes the kernel function $\rho$ for which $I_{\rho}$ is bounded from $L^{p}\left(\mathbb{R}^{n}\right)$ to $L^{q}\left(\mathbb{R}^{n}\right)$ for $1<p<q<\infty$. We have the following result: 
Corollary 4.60. [18, Corollary 1.5] Let $1<p<q<\infty$. The operator $I_{\rho}$ is bounded from $L^{p}\left(\mathbb{R}^{n}\right)$ to $L^{q}\left(\mathbb{R}^{n}\right)$ if and only if $\rho(r) \lesssim r^{\frac{n}{p}-\frac{n}{q}}$ for all $r>0$.

For $\rho(r)=r^{\alpha}$, Corollary 4.60 further reads that the operator $I_{\rho}$ is bounded from $L^{p}\left(\mathbb{R}^{n}\right)$ to $L^{q}\left(\mathbb{R}^{n}\right)$ if and only if $\alpha=\frac{n}{p}-\frac{n}{q}$, where $1<p<q<\infty$.

With Theorems 4.53-4.57 we can characterize the function $\rho$ for which $I_{\rho}$ is bounded from one Morrey space to another.

The next corollary generalizes the previous characterization in Corollary 4.60.

Corollary 4.61. [18, Corollary 1.6] Assume that the parameters $p, q, s, t$ and $\alpha$ satisfy

$$
1<q \leq p<\infty, \quad 1<t \leq s<\infty, \quad 0<\alpha<n
$$

and

$$
\frac{1}{s}=\frac{1}{p}-\frac{\alpha}{n}, \quad \frac{t}{s}=\frac{q}{p} .
$$

Let $\rho:(0, \infty) \rightarrow(0, \infty)$ be a function satisfying the growth condition. Then the generalized fractional integral operator $I_{\rho}$ is bounded from $\mathcal{M}_{q}^{p}\left(\mathbb{R}^{n}\right)$ to $\mathcal{M}_{t}^{s}\left(\mathbb{R}^{n}\right)$ precisely when $\rho(r) \lesssim r^{\alpha}$.

We show by examples that two statements in Theorem 4.57 are of independent interest. As before we write

$$
\ell^{\mathbb{B}}(r) \equiv \begin{cases}(1+|\log r|)^{\beta_{1}} & (0<r \leq 1), \\ (1+|\log r|)^{\beta_{2}} & (1<r<\infty) .\end{cases}
$$

This function is used to describe the "log"-growth and "log"-decay properties. Also, we fix $p$ and $q$ so that $1<p<q<\infty$. The key properties we are interested in are summarized in the following table:

\begin{tabular}{llll} 
& $\frac{\rho}{\varphi} \in \mathbb{Z}_{0} \varphi \in \mathbb{Z}^{-\frac{n}{p}}$ & $(69)$ & $(70)$ \\
\hline \hline Example 4.62 + & + & + & + \\
\hline Example 4.63 + & - & + & + \\
\hline Example 4.64 - & - & + & + \\
\hline Example 4.65 - & - & + & + \\
\hline
\end{tabular}

Example 4.62. [18, Example 2] Let $\lambda<0$ satisfy $0<\left(\frac{p}{q}-1\right) \lambda<n$ and $-\frac{n}{p}<\lambda$. Take $\mu_{1}, \mu_{2} \in \mathbb{R}$ arbitrarily. Set $\beta_{i} \equiv\left(\frac{p}{q}-1\right) \mu_{i}$ for $i=1,2$. Define $\varphi(r) \equiv$ $r^{-\lambda} \ell_{-\mu_{1}-, \mu_{2}}(r)$ and $\rho(r)=\varphi(r)^{1-\frac{p}{q}}$ for $r>0$. Then this pair $(\rho, \varphi)$ fulfills the assumptions $\frac{\rho}{\varphi} \in \mathbb{Z}_{0}$ and $\varphi \in \mathbb{Z}^{-\frac{n}{p}}$ in Theorem 4.57. Indeed, for $r>0$ we have $\tilde{\rho}(r) \sim \rho(r)=\varphi(r)^{1-\frac{p}{q}}$ and

$$
\int_{r}^{\infty} \frac{\rho(t)}{t \varphi(t)} d t \sim \frac{\rho(r)}{\varphi(r)}
$$

Example 4.63 is an endpoint case of the above example. 
Example 4.63. [18, Example 1] Let $\mu_{1}, \mu_{2} \geq 0$. Set $\alpha \equiv \frac{n}{p}-\frac{n}{q}$ and $\beta_{i} \equiv\left(\frac{p}{q}-1\right) \mu_{i}$ for $i=1,2$. Define $\varphi(r) \equiv r^{\frac{n}{q}} \ell_{-\mu_{1},-\mu_{2}}(r)$ for $r>0$ and $\rho=\varphi^{1-\frac{p}{q}}$. We note that $\tilde{\rho} \sim \rho$. Then this pair $(\rho, \varphi)$ fulfills the assumptions $\frac{\rho}{\varphi}=\varphi^{-\frac{p}{q}} \in \mathbb{Z}_{0}$ and (69) but $\varphi \notin \mathbb{Z}^{-\frac{n}{p}}$ since $\ell_{-\mu_{1},-\mu_{2}} \notin \mathbb{Z}^{0}$.

The next example concerns the case where the spaces are close to $L^{\infty}\left(\mathbb{R}^{n}\right)$ and the smoothing order of $I_{\rho}$ is "almost 0 ".

Example 4.64. [18, Example 3] Let $\mu_{1}, \mu_{2}<0$. Set $\beta_{1} \equiv\left(\frac{p}{q}-1\right) \mu_{1}+1 \in(1, \infty)$ and $\beta_{2} \equiv\left(\frac{p}{q}-1\right) \mu_{2}-1 \in(-1, \infty)$. Define $\rho \equiv \ell^{\mathbb{B}}$ as we did in Example 3.10 and let $\varphi \equiv \ell_{\mu_{1}, \mu_{2}}$. Then this pair $(\rho, \varphi)$ fulfills $\varphi \notin \mathbb{Z}^{-\frac{n}{p}}$ and assumption (69) but $\frac{\rho}{\varphi}=\ell_{\beta_{1}-\mu_{1}, \beta_{2}-\mu_{2}} \notin \mathbb{Z}_{0}$. More precisely, we have $\tilde{\rho} \sim \ell_{\beta_{1}-1, \beta_{2}+1}$ since $\beta_{1}>1$, and

$$
\int_{r}^{\infty} \frac{\rho(t)}{t \varphi(t)} d t \sim \ell_{\mu_{1}+\beta_{1}-1, \mu_{2}+\beta_{2}+1}(r) \quad(r>0) .
$$

We consider a case where the target space is close to $L^{\infty}\left(\mathbb{R}^{n}\right)$.

Example 4.65. [18, Example 4] Let $1<p, q<\infty$. Let $\alpha, \beta_{1}, \mu_{1}, \mu_{2}$ satisfy $0<$ $\alpha<\frac{n}{p}, \mu_{1}+\beta_{1}<1, \mu_{2}<0$. Set $\beta_{2} \equiv\left(\frac{p}{q}-1\right) \mu_{2}-1 \in(-1, \infty)$. Define $\rho(r) \equiv$ $\min \left(1, r^{\alpha}\right) \ell^{\mathbb{B}}(r)$ as we did in Example 3.10 and let $\varphi(r) \equiv \max \left(1, r^{-\alpha}\right) \ell_{\mu_{1}, \mu_{2}}(r)$ for $r>0$. Then this pair $(\rho, \varphi)$ fulfills $\varphi \notin \mathbb{Z}^{-\frac{n}{p}}$ and assumption (69) but $\frac{\rho}{\varphi} \notin \mathbb{Z}_{0}$ More precisely,

and

$$
\frac{\tilde{\rho}(r)}{\varphi(r)} \sim \ell_{\mu_{1}+\beta_{1}, \mu_{2}+\beta_{2}+1}(r)
$$

for $r>0$.

$$
\int_{r}^{\infty} \frac{\rho(t)}{t \varphi(t)} d t \sim \ell_{\mu_{1}+\beta_{1}-1, \mu_{2}+\beta_{2}+1}(r)
$$

Based upon these preliminary results and Lemma 4.51, we will prove Theorems 4.57-4.53.

We remark that (69) includes (70). We prove an estimate. Once we prove Lemma 4.59 below, we can obtain the boundedness of $I_{\rho}$ from $\mathcal{M}_{p}^{\varphi}\left(\mathbb{R}^{n}\right)$ to $\mathcal{M}_{q}^{\varphi^{p / q}}\left(\mathbb{R}^{n}\right)$ as we will see below. Here we use the fact that the Hardy-Littlewood maximal operator $M$ is bounded on $\mathcal{M}_{p}^{\varphi}\left(\mathbb{R}^{n}\right)$, if $p>1$ and $\varphi$ is almost decreasing; see Theorem 4.1 .

We end this section with comparison of our results with the existing results. We move on to the case of Spanne type.

Remark 4.66. See [29, Theorem 5.2] for the Spanne-type boundedness of $I_{\alpha}$. See [33, Theorem 5.4], [107, Theorem 1.8], [108, Theorems 2.6, 2.7, 3.4 and 3.6] and [120, Theorem 1.5] for the case of the multilinear setting.

Remark 4.67. See [110, Theorem 2.3] for the weak boundedness of the maximal operators (on nonhomogeneous spaces), where the integral conditions is assumed. 
Remark 4.68. See [29, Theorem 5.5] and [47, Theorem 5.7] for the Adams-type boundedness of $I_{\alpha}$. Persson and Samko obtainted the Adams-type boundedness of $I_{\alpha}$ using the weighted Hardy operator; see [86, Theorem 5.4]. Guliyev and Shukurov also considered a similar situation in [46, Theorem 3.6] and [47, Theorem 5.7]. See [7, Theorem 2] for the multilinear case.

\section{Remark 4.69.}

- See [73, Theorem 3], [50] for the boundedness of $I_{\alpha}$ on generalized Morrey spaces.

- See [75, Theorem 7.1] for the boundedness of the generalized fractional integral operators on generalized Orlicz Morrey spaces (of the first kind).

- See $[17,49,50,70,117,118]$ for the study of the boundedness of $I_{\rho}$.

- See [94] for a different type of generalization of the form:

$$
I f(x)=\int_{\mathbb{R}^{n}} K(x, y) f(y) d y .
$$

Remark 4.70. In some special case, some authors obtained the necessity of the boundedness of $I_{\alpha}=I_{\rho}$ from generalized Morrey spaces to other generalized Morrey spaces. See [19, Theorems 2.3 and 3.2] as well as [49], [101].

Remark 4.71. Kurata and Sugano pointed out that the operator of the form $V^{\gamma}(-\Delta+V)^{\beta}$ with a potentail $V$ satisfying the reverse Hölder inequality falls under the scope of the results in this section [62]. Here $\beta, \gamma$ are suitable real parameters. See [62] for more details.

Remark 4.72. Many researchers handled various operators.

- In [15] Eroglu dealt with fractional oscillatory integral operators and their commutators.

- In [63] Liu and Shi considered the boundedness of the commutator generated by BMO and the fractional integral operators. See also [33, Theorem 7.1], [47, Theorem 7.11] for the Spanne type result and [47, Theorem 7.13] for the Adams type result.

4.4. Generalized fractional maximal operators in generalized Morrey spaces. We discuss the boundedness property of the generalized fractional maximal operator, defined by:

$$
M_{\rho} f(x)=\sup _{r>0} \frac{\rho(r)}{|B(x, r)|} \int_{B(x, r)}|f(y)| d y \quad\left(x \in \mathbb{R}^{n}\right),
$$

where $f \in L_{\text {loc }}^{1}\left(\mathbb{R}^{n}\right)$ and $\rho$ is a suitable function from $(0, \infty)$ to $[0, \infty)$.

Example 4.73. Let $0 \leq \alpha<n$.

- If we let $\rho(t)=t^{\alpha}$, then we obtain the fractional maximal operator $M_{\alpha}$; $M_{\rho}=M_{\alpha}$. 
- If we let $\rho(t)=\min \left(t^{\alpha}, 1\right)$, then we obtain the local fractional maximal operator $m_{\alpha} ; M_{\rho}=m_{\alpha}$, where

$$
m_{\alpha} f(x)=\sup _{0<r \leq 1} \frac{r^{\alpha}}{|B(x, r)|} \int_{B(x, r)}|f(y)| d y
$$

What $M_{\rho}$ is to $I_{\rho}$ is what $M_{\alpha}$ is to $I_{\alpha}$. So, we are interested in when $M_{\rho}$ is bounded from $\mathcal{M}_{q}^{\varphi}\left(\mathbb{R}^{n}\right)$ to $\mathcal{M}_{t}^{\psi}\left(\mathbb{R}^{n}\right)$. We start with the following necessary condition:

Proposition 4.74. [51, Theorem 1] Let $1 \leq q<\infty$ and $(\varphi, \psi) \in \mathcal{G}_{q} \times \mathcal{G}_{1}$. Assume that $M_{\rho}$ is bounded from $\mathcal{M}_{q}^{\varphi}\left(\mathbb{R}^{n}\right)$ to $\mathrm{w} \mathcal{M}_{1}^{\psi}\left(\mathbb{R}^{n}\right)$. Then $\rho \lesssim \frac{\varphi}{\psi}$. In particular, if $M_{\rho}$ is bounded from $\mathcal{M}_{q}^{\varphi}\left(\mathbb{R}^{n}\right)$ to $\mathcal{M}_{1}^{\psi}\left(\mathbb{R}^{n}\right)$, then $\rho \lesssim \frac{\varphi}{\psi}$.

Proof. Let $R>0$ be fixed. We utilize the pointwise estimate $\rho(R) \chi_{B(R)} \leq M_{\rho} \chi_{B(2 R)}$, and the doubling condition of $\varphi$ to obtain

$$
\rho(R) \lesssim \frac{\left\|\rho(R) \chi_{B(R)}\right\|_{\mathrm{w} \mathcal{M}_{1}^{\psi}}}{\psi(R)} \lesssim \frac{\left\|M_{\rho} \chi_{B(2 R)}\right\|_{\mathcal{M}_{1}^{\psi}}}{\psi(R)} \lesssim \frac{\left\|\chi_{B(2 R)}\right\|_{\mathcal{M}_{q}^{\varphi}}}{\psi(R)} \sim \frac{\varphi(R)}{\psi(R)}
$$

Our first result completely characterizes the boundedness of $M_{\rho}$ on generalized Orlicz-Morrey spaces.

Theorem 4.75. [51, Theorem 1] Let $0<a<1<q<\infty$. Let $\varphi \in \mathcal{G}_{q}$. Then, $M_{\rho}$ is bounded from $\mathcal{M}_{q}^{\varphi}\left(\mathbb{R}^{n}\right)$ to $\mathcal{M}_{a^{-1} q}^{\varphi^{a}}\left(\mathbb{R}^{n}\right)$ if and only if $\rho$ and $\varphi$ satisfy the inequality

$$
\rho(R) \lesssim \varphi(R)^{1-a}
$$

for all $R>0$.

The proof hinges on the following Hedberg inequality:

Lemma 4.76. [51, (15)] Let $0<a<1<q<\infty$. Let $\varphi \in \mathcal{G}_{q}$. Then for any $f \in \mathcal{M}_{q}^{\varphi}\left(\mathbb{R}^{n}\right)$ with $\|f\|_{\mathcal{M}_{q}^{\varphi}} \leq 1$,

$$
M_{\rho} f(x) \lesssim M f(x)^{a} \quad\left(x \in \mathbb{R}^{n}\right)
$$

Once Lemma 4.76 is proved, we have only to resort to the scaling law (Lemma 3.11) and the boundedness of $M$ on $\mathcal{M}_{q}^{\varphi}\left(\mathbb{R}^{n}\right)$.

Proof of Lemma 4.76. Remark that both $\varphi$ is bijective. Let $R>0$. By using the definition of $M$, we obtain

$$
\frac{\rho(R)}{|B(x, R)|} \int_{B(x, R)}|f(y)| d y \leq \rho(R) M f(x) \lesssim \varphi(R)^{1-a} M f(x)
$$

and

$$
\frac{\rho(R)}{|B(x, R)|} \int_{B(x, R)}|f(y)| d y \lesssim \rho(R) \frac{\|f\|_{\mathcal{M}_{1}^{\varphi}}}{\varphi(R)} \lesssim \varphi(R)^{-a}
$$


Thus, it follows that

$$
\begin{aligned}
\frac{\rho(R)}{|B(x, R)|} \int_{B(x, R)}|f(y)| d y & \lesssim \min \left\{\varphi(R)^{1-a} M f(x), \varphi(R)^{-a}\right\} \\
& \leq \sup _{t>0} \min \left\{t^{1-a} M f(x), t^{-a}\right\} \\
& =M f(x)^{a} .
\end{aligned}
$$

Since $R>0$ being arbitrary, we obtain (75).

The weak boundedness of $M_{\rho}$ can be characterized in a similar way.

Corollary 4.77. [51, Corollary 1] Let $0<a<1 \leq q<\infty$. Let $\varphi \in \mathcal{G}_{q}$. Then, $M_{\rho}$ is bounded from $\mathcal{M}_{q}^{\varphi}\left(\mathbb{R}^{n}\right)$ to $\mathrm{w} \mathcal{M}_{a^{-1} q}^{\varphi^{a}}\left(\mathbb{R}^{n}\right)$ if and only if $\rho$ and $\varphi$ satisfy (74) for all $R>0$.

We move on to the vector-valued inequality for $M_{\rho}$ on generalized OrliczMorrey spaces and generalized weak Orlicz-Morrey spaces.

Theorem 4.78. [51, Theorem 8] Let $0<a<1<q<\infty$ and let $1 \leq u<\infty$. Let $\varphi \in \mathcal{G}_{q}$.

- If $\rho$ and $\varphi$ satisfy (33) and (74), then for $\left\{f_{j}\right\}_{j=1}^{\infty} \subset \mathcal{M}_{q}^{\varphi}\left(\mathbb{R}^{n}\right)$

$$
\left\|\left(\sum_{j=1}^{\infty} M_{\rho} f_{j}^{u}\right)^{\frac{1}{u}}\right\|_{\mathcal{M}_{a^{-1} q}^{\varphi^{a}}} \lesssim\left\|\left(\sum_{j=1}^{\infty}\left|f_{j}\right|^{u}\right)^{\frac{1}{u}}\right\|_{\mathcal{M}_{q}^{\varphi}} .
$$

- Conversely, if

$$
\left\|\left(\sum_{j=1}^{\infty} M_{\rho} f_{j}{ }^{u}\right)^{\frac{1}{u}}\right\|_{\mathrm{w}_{\mathcal{M}_{1}^{\varphi}}{ }^{a}} \lesssim\left\|\left(\sum_{j=1}^{\infty}\left|f_{j}\right|^{u}\right)^{\frac{1}{u}}\right\|_{\mathcal{M}_{q}^{\varphi}}
$$

for $\left\{f_{j}\right\}_{j=1}^{\infty} \subset \mathcal{M}_{q}^{\varphi}\left(\mathbb{R}^{n}\right)$, then $\rho, \varphi$ and $\psi$ satisfy (74). Moreover, under the assumption that $\rho \sim \varphi / \psi$, inequality (76) holds if and only if $\varphi$ satisfies (33).

Proof.

- Using (74), we may assume that $\rho=\varphi^{1-a}$. Then since $\varphi$ is a doubling function and $0<a<1$, we have

$$
M_{\varphi^{1-a}} f_{j} \lesssim I_{\varphi^{1-a}}\left|f_{j}\right| .
$$

Thus,

$$
\left(\sum_{j=1}^{\infty} M_{\rho} f_{j}{ }^{u}\right)^{\frac{1}{u}} \lesssim\left(\sum_{j=1}^{\infty}\left(I_{\varphi^{1-a}}\left|f_{j}\right|\right)^{u}\right)^{\frac{1}{u}} \lesssim I_{\varphi^{1-a}}\left[\left(\sum_{j=1}^{\infty}\left|f_{j}\right|^{u}\right)^{\frac{1}{u}}\right]
$$


Since we can verify (69), it remains to resort to the boundedness of $I_{\varphi^{1-a}}$ from $\mathcal{M}_{q}^{\varphi}\left(\mathbb{R}^{n}\right)$ to $\mathcal{M}_{a^{-1} q}^{\varphi^{a}}\left(\mathbb{R}^{n}\right)$.

- We let

$$
f_{j} \equiv\left\{\begin{array}{ll}
f, & (j=1), \\
0, & (j \geq 1),
\end{array} \quad f \in \mathcal{M}_{1}^{\varphi}\left(\mathbb{R}^{n}\right) .\right.
$$

Then we have the boundedness of $M_{\rho}$ on $\mathcal{M}_{1}^{\varphi}\left(\mathbb{R}^{n}\right)$. Hence, by Theorem 4.75 , we conclude that the inequality (74) holds.

Finally, under the assumption that $\rho \sim \varphi / \psi$, we prove that inequality (76) holds if and only if $\varphi$ satisfies (33). To do this, it is enough to show that (33) follows from (76). Now, assume that the integral condition (33) fails. Then, for any $m \in \mathbb{N}$, there exists $r_{m}>0$ such that

$$
\varphi\left(2^{m} r_{m}\right) \leq 2 \varphi\left(r_{m}\right) .
$$

Letting $f_{j}=\chi_{[1, m]}(j) \chi_{B\left(2^{j} r_{m}\right) \backslash B\left(2^{j-1} r_{m}\right)}, j \in \mathbb{N}$, we have

$$
\left\|f_{j}\right\|_{\mathcal{M}_{\Phi}^{\varphi}\left(\ell^{u}\right)} \leq\left\|\chi_{B\left(2^{m} r_{m}\right)}\right\|_{\mathcal{M}_{\Phi}^{\varphi}} \sim \varphi\left(2^{m} r_{m}\right) \leq 2 \varphi\left(r_{m}\right) .
$$

Since $\theta \in \mathcal{G}_{n}$ and $\rho \sim \varphi / \psi=\varphi / \theta(\varphi), \rho(r) \lesssim \rho(s)$ for all $r \leq s$. Due to this fact and the inequality $M_{\rho} f_{j} \gtrsim \rho\left(2^{j} r_{m}\right) \chi_{B\left(r_{m}\right)}$, we have

$$
\begin{aligned}
\left\|M_{\rho} f_{j}\right\|_{\mathrm{w} \mathcal{M}_{1}^{\psi}\left(\ell^{u}\right)} & \gtrsim\left\|\left(\sum_{j=1}^{m} \rho\left(2^{j} r_{m}\right)^{u}\right)^{\frac{1}{u}} \chi_{B\left(r_{m}\right)}\right\|_{\mathrm{w} \mathcal{M}_{1}^{\psi}} \\
& \gtrsim \rho\left(2 r_{m}\right) \psi\left(2 r_{m}\right) m^{\frac{1}{u}} \\
& \gtrsim \varphi\left(r_{m}\right) m^{\frac{1}{u}} .
\end{aligned}
$$

We combine the inequalities (77) and (78) with the boundedness of $M_{\rho}$ from $\mathrm{w}_{\mathcal{q}}^{\varphi}\left(\ell^{u}\right)$ to $\mathcal{M}_{1}^{\psi}\left(\ell^{u}\right)$ to obtain $m \leq D$ where $D$ is independent of $m$, contradictory to the fact that $m \in \mathbb{N}$ is arbitrary. Thus the integral condition (33) holds.

Remark 4.79. One may ask ourselves how different $I_{\alpha}$ and $M_{\alpha}$. See $[27$, Theorem 1.10] or compare [101, Theorem 1.3] with [101, Theorem 1.7] and [101, Proposition 4.1] to see the gap between $I_{\alpha}$ and $M_{\alpha}$. See also [27, Theorems 5.1 and 5.2] to see that when they are the same.

Remark 4.80. See [29, Theorem 5.2] and [47, Theorem 4.3] for the Spanne-type boundedness of $M_{\alpha}$, where the integral condition is assumed.

Remark 4.81. See [29, Theorem 5.5] and [47, Theorem 4.4] for the Adams-type boundedness of $M_{\alpha}$, where the integral condition is assumed.

Remark 4.82. See [127, Theorem 4.1] for the boundedness of the fractional maximal operator on generalized Morrey spaces in the multilinear setting. 


\section{OVERVIEW OF OTHER TYPES OF GENERALIZATIONS}

5.1. Morrey spaces for general Radon measures and Morrey spaces over metric measure spaces. In addition to the genealization of $p$ into functions, one can replace the Lebesgue measure by general Radon measures. Here we work on a metric measure space $(X, d, \mu)$. We refer to [125] for an exhaustive account of the analysis on metric measure spaces. Let $k>0$ and $0<q \leq p<\infty$. We define the Morrey space $\mathcal{M}_{q}^{p}(k, \mu)$ as

$$
\mathcal{M}_{q}^{p}(k, \mu) \equiv\left\{f \in L_{\mathrm{loc}}^{q}(\mu):\|f\|_{\mathcal{M}_{q}^{p}(k, \mu)}<\infty\right\},
$$

where

$$
\|f\|_{\mathcal{M}_{q}^{p}(k, \mu)} \equiv \sup _{B(x, r) \in \mathcal{B}(\mu)} \mu(B(x, k r))^{\frac{1}{p}-\frac{1}{q}}\left(\int_{B(x, r)}|f(z)|^{q} d \mu(z)\right)^{\frac{1}{q}} .
$$

Here $\mathcal{B}(\mu)$ stands for the set of all balls having positve $\mu$-measure. In the Euclidean space $\mathbb{R}^{n}, \mathcal{Q}(\mu)$ stands for the set of all cubes having positve $\mu$-measure. Clearly we have $L^{p}(\mu)=\mathcal{M}_{p}^{p}(k, \mu)$, and by applying Hölder's inequality to (79) we have $\|f\|_{\mathcal{M}_{q_{1}}^{p}(k, \mu)} \geq\|f\|_{\mathcal{M}_{q_{2}}^{p}(k, \mu)}$ for all $p \geq q_{1} \geq q_{2}>0$ and $k \geq 1$. Thus the following inclusions hold:

Proposition 5.1. Let $p \geq q_{1} \geq q_{2}>0$ and $k \geq 1$. Then

$$
L^{p}(\mu)=\mathcal{M}_{p}^{p}(k, \mu) \subset \mathcal{M}_{q_{1}}^{p}(k, \mu) \subset \mathcal{M}_{q_{2}}^{p}(k, \mu) .
$$

A remarkable property of $\mathcal{M}_{q}^{p}(k, \mu)$ is that the space $\mathcal{M}_{q}^{p}(k, \mu)$ does not depend on $k>1$.

Proposition 5.2. Let $(X, d, \mu)$ be the Euclidean space $\mathbb{R}^{n}$ with the Euclidean distance and the Lebesgue measure. Then for all $\mathcal{M}_{q}^{p}(k, \mu)=\mathcal{M}_{q}^{p}(2, \mu) p \geq q>0$ and $k>1$.

We do not recall its proof whose proof hinges on a geometric structure of $\mathbb{R}^{n}$; see [104, Proposition 1.1]. It can happen that $\mathcal{M}_{q}^{p}(1, \mu)$ is a proper subset $\mathcal{M}_{q}^{p}(2, \mu)$ in Proposition 5.2, as was shown in [99]. Instead of the norm above, we can use

$$
\|f\|_{\mathcal{L}_{q}^{\nu}(k, \mu)} \equiv \sup _{B(x, r) \in \mathcal{B}(\mu)} r^{\nu} \mu(B(x, k r))^{-\frac{1}{q}}\left(\int_{B(x, r)}|f(z)|^{q} d \mu(z)\right)^{\frac{1}{q}} .
$$

See [72]. See also [95]. We can also define the generalized Morrey spaces with Radon measures. We work on a metric measure space $(X, d, \mu)$. Let $\varphi:(0, \infty) \rightarrow(0, \infty)$ be a function. Then define

$$
\mathcal{M}_{q}^{\varphi}(k, \mu) \equiv\left\{f \in L_{\mathrm{loc}}^{q}(\mu):\|f\|_{\mathcal{M}_{q}^{\varphi}(k, \mu)}<\infty\right\}
$$

where

$$
\|f\|_{\mathcal{M}_{q}^{p}(k, \mu)} \equiv \sup _{B(x, r) \in \mathcal{B}(\mu)} \varphi(\mu(B(x, k r)))\left(\frac{1}{\mu(B(x, k r))} \int_{B(x, r)}|f(z)|^{q} d \mu(z)\right)^{\frac{1}{q}} .
$$


See [45, 91] for generalized Morrey spaces with general Radon measures and [90] for weak Morrey spaces with general Radon measures. See [56, 53, 93, 96, 97] for this direction of approaches. We refer to [126] for Morrey spaces with gradient in this type of setting.

We can consider some concrete cases. See $[20,34,39]$ for generalized Morrey spaces on Heisenberg group, [48] for parabolic genealized Morrey spaces and [13, 40] for anisotropic genealized Morrey spaces. See [16, 32] and [121] for generalized Morrey spaces on Carnot groups and for generalized Morrey spaces for the $p$-adic fields.

5.2. Local generalized Morrey spaces. Motivated by the works [28, 43], define the generalized local Morrey space $\operatorname{LM}_{q}^{\varphi}\left(\mathbb{R}^{n}\right)$ to be the set of all measurable functions $f$ such that

$$
\|f\|_{\mathrm{L} \mathcal{M}_{q}^{\varphi}} \equiv \sup _{r>0} \varphi(r)\left(\frac{1}{|Q(r)|} \int_{Q(r)}|f(y)|^{q} d y\right)^{\frac{1}{q}}<\infty .
$$

The space $\operatorname{L} \mathcal{M}_{q}^{\varphi}\left(\mathbb{R}^{n}\right)$ is sometimes referred to as the $B_{\sigma}$-spaces. See $[78,106]$ for $B_{\sigma}$-spaces.

5.3. Generalized Orlicz-Morrey spaces. Instead of $p$, we can generalize $q$ using Young functions. In the definition below we exclude the case where $\varphi(t)=\infty$ for some $t \in(0, \infty)$.

Definition 5.3 (Young function). A function $\Phi:[0, \infty) \rightarrow[0, \infty)$ is said to be a Young function, if there exists an increasing function $\varphi$ which is right-continuous such that

$$
\Phi(t)=\int_{0}^{t} \varphi(s) \mathrm{d} s \quad(t \geq 0) .
$$

Equality (82) is called the canonical representation of a Young function $\Phi$. By convention define $\Phi(\infty) \equiv \infty$.

There are three types of generalizations. We start with the Orlicz-Morrey spaces of the first kind. To this end we start with the defintion of the $(\varphi, \Phi)$-average over $Q$.

Definition $5.4((\varphi, \Phi)$-average $)$. Let $\varphi:(0, \infty) \rightarrow(0, \infty)$ be a function and $\Phi$ : $(0, \infty) \rightarrow(0, \infty)$ a Young function. For a cube $Q$ and $f \in L^{0}(Q)$, define the $(\varphi, \Phi)$-average over $Q$ by:

$$
\|f\|_{(\varphi, \Phi) ; Q} \equiv \inf \left\{\lambda>0: \frac{\varphi(\ell(Q))}{|Q|} \int_{Q} \Phi\left(\frac{|f(x)|}{\lambda}\right) d x \leq 1\right\} .
$$

In [75] Nakai defined the generalized Orlicz-Morrey space of the first kind via the $(\varphi, \Phi)$-average.

Definition 5.5 (Generalized Orlicz-Morrey spaces of the first kind). Suppose thatwe have a function $\varphi:(0, \infty) \rightarrow(0, \infty)$ and a Young function $\Phi:[0, \infty) \rightarrow$ 
$[0, \infty)$. For a measurable function $f$ define $\|f\|_{\mathcal{L}_{\Phi}^{\varphi}} \equiv \sup _{Q \in \mathcal{Q}}\|f\|_{(\varphi, \Phi) ; Q}$. The function space $\mathcal{L}_{\Phi}^{\varphi}\left(\mathbb{R}^{n}\right)$, the generalized Orlicz-Morrey space of the first kind, is defined to be the set of all measurable functions $f$ for which the norm $\|f\|_{\mathcal{L}_{\Phi}^{\varphi}}$ is finite. Likewise the function space $\mathrm{W} \mathcal{L}_{\Phi}^{\varphi}\left(\mathbb{R}^{n}\right)$, the weak generalized Orlicz-Morrey space of the first kind, is defined to be the set of all measurable functions $f$ for which the norm $\|f\|_{\mathrm{W} \mathcal{L}_{\Phi}^{\varphi}}=\sup _{\lambda>0} \lambda\left\|\chi_{(\lambda, \infty]}(|f|)\right\|_{\mathcal{L}_{\Phi}^{\varphi}}$ is finite.

We move on to Orlicz-Morrey spaces of the second kind. To define OrliczMorrey spaces of the second kind, we need another notion of the average.

Definition 5.6. Let $\Phi:[0, \infty) \rightarrow(0, \infty)$ a Young function. For a cube $Q$, define its $\Phi$-average over $Q$ by:

$$
\|f\|_{\Phi ; Q} \equiv \inf \left\{\lambda>0: \frac{1}{|Q|} \int_{Q} \Phi\left(\frac{|f(x)|}{\lambda}\right) d x \leq 1\right\} .
$$

With this new definition of the average in mind, Sawano, Sugano and Tanaka define generalized Orlicz-Morrey spaces of the second kind [103].

Definition 5.7 (Generalized Orlicz-Morrey spaces of the second kind). Suppose that we have a Young function $\Phi:[0, \infty) \rightarrow[0, \infty)$ and a function $\varphi:(0, \infty) \rightarrow$ $[0, \infty)$. Let $f \in L^{0}\left(\mathbb{R}^{n}\right)$.

- Define $\|f\|_{\mathcal{M}_{\Phi}^{\varphi}} \equiv \sup _{Q \in \mathcal{Q}} \varphi(\ell(Q))\|f\|_{\Phi ; Q}$. The generalized Orlicz-Morrey space of the second kind $\mathcal{M}_{\Phi}^{\varphi}\left(\mathbb{R}^{n}\right)$ is defined to be the the set of all measurable functions $f$ for which the norm $\|f\|_{\mathcal{M}_{\Phi}^{\varphi}}$ is finite.

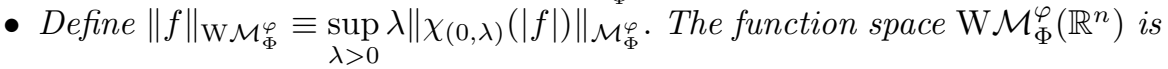
defined to be the weak generalized Orlicz-Morrey space of the second kind as the set of all measurable functions f for which the norm $\|f\|_{\mathrm{WM}}^{\varphi}$ is finite.

Finally, Deringoz, Samko and Guliyev define generalized Orlicz-Morrey space of the third kind as follows:

Definition 5.8. The generalized Orlicz-Morrey space $\mathcal{Z}_{\Phi}^{\varphi}\left(\mathbb{R}^{n}\right)$ of the third kind is defined as the set of all measurable functions $f$ for which the norm

$$
\|f\|_{\mathcal{Z}_{\Phi}^{\varphi}} \equiv \sup _{Q \in \mathcal{Q}} \varphi(\ell(Q)) \Phi^{-1}\left(\frac{1}{|Q|}\right)\|f\|_{L^{\Phi}(Q)}
$$

is finite.

We do not go into the details of these function spaces; here we content ourselves with mentioning that the first kind and the second kind are different and that the second kind and the third kind are different according to [22]. See $[11,36,52,98,102,119]$ for more about these function spaces. 
5.4. Generalization of $\varphi$ to the function depending also on $x$. One can consider the case where $\varphi$ depends on $x$ not only on $r$.

$$
\|f\|_{\mathcal{M}_{q}^{\varphi}} \equiv \sup _{x \in \mathbb{R}^{n}, r>0} \varphi(x, r)\left(\frac{1}{|Q(x, r)|} \int_{Q(x, r)}|f(y)|^{q} d y\right)^{\frac{1}{q}}<\infty .
$$

See $[4,29,45,73]$ for the approach to this direction.

5.5. Martingale Morrey spaces. One can use martingales to generalize Morrey spaces $[77,81]$.

Let $(\Omega, \Sigma, P)$ be a probability space, and $\mathcal{F}=\left\{\mathcal{F}_{n}\right\}_{n>0}$ a nondecreasing sequence of sub- $\sigma$-algebras of $\Sigma$ such that $\Sigma=\sigma\left(\bigcup_{n} \mathcal{F}_{n}\right)$. For the sake of simplicity, let $\mathcal{F}_{-1}=\mathcal{F}_{0}$. The set $B \in \mathcal{F}_{n}$ is called atom, more precisely $\left(\mathcal{F}_{n}, P\right)$-atom, if any $A \subset B, A \in \mathcal{F}_{n}$, satisfies $P(A)=P(B)$ or $P(A)=0$. Denote by $A\left(\mathcal{F}_{n}\right)$ the set of all atoms in $\mathcal{F}_{n}$.

The expectation operator and the conditional expectation operators relative to $\mathcal{F}_{n}$ are denoted by $E$ and $E_{n}$, respectively. It is known as the Doob theorem that, if $p \in(1, \infty)$, then any $L_{p}$-bounded martingale converges in $L_{p}$. Moreover, if $p \in[1, \infty)$, then, for any $f \in L_{p}$, its corresponding martingale $\left\{f_{n}\right\}_{n=1}^{\infty}$ with $f_{n}=E_{n} f$ is an $L_{p}$-bounded martingale and converges to $f$ in $L_{p}$ (see for example [82]). For this reason a function $f \in L_{1}$ and the corresponding martingale $\left\{f_{n}\right\}_{n=1}^{\infty}$ will be denoted by the same symbol $f$.

Let $\mathcal{M}$ be the set of all martingales such that $f_{0}=0$. For $p \in[1, \infty]$, let $L_{p}^{0}(\Omega, \mathcal{F}, P)$ be the set of all $f \in L^{p}(\Omega, \mathcal{F}, P)$ such that $E_{0} f=0$. For any $f \in$ $L_{p}^{0}(\Omega, \mathcal{F}, P)$, its corresponding martingale $\left(f_{n}\right)$ with $f_{n}=E_{n} f$ is an $L_{p}$-bounded martingale in $\mathcal{M}$. For this reason we regard as $L_{p}^{0}(\Omega, \mathcal{F}, P) \subset \mathcal{M}$.

Let $\mathcal{B}=\left\{\mathcal{B}_{n}\right\}_{n \geq 0}$ be sub-families of $\mathcal{F}=\left\{\mathcal{F}_{n}\right\}_{n \geq 0}$ with $\mathcal{B}_{n} \subset \mathcal{F}_{n}$ for each $n \geq 0$. We denote by $\mathcal{B} \subset \mathcal{F}$ this relation of $\mathcal{B}$ and $\mathcal{F}$.

In this paper we always postulate the following condition on $\mathcal{B}$ :

There exists a countable subset $\mathcal{B}^{\prime} \subset \mathcal{B}_{0}$ such that $P\left(\bigcup_{B \in \mathcal{B}^{\prime}} B\right)=1$.

We first define generalized martingale Morrey-Campanato spaces with respect to $\mathcal{B}$ as the following: 
Definition 5.9. Let $\mathcal{B} \subset \mathcal{F}, p \in[1, \infty)$ and $\varphi:(0,1] \rightarrow(0, \infty)$. For $f \in L_{1}$, let

$$
\begin{aligned}
\|f\|_{L_{p}^{\varphi}} & =\|f\|_{L_{p}^{\varphi}(\mathcal{B})} \\
& =\sup _{n \geq 0} \sup _{B \in \mathcal{B}_{n}} \varphi(P(B))\left(\frac{1}{P(B)} \int_{B}|f(\omega)|^{p} d P(\omega)\right)^{1 / p}, \\
\|f\|_{\mathcal{L}_{p}^{\varphi}} & =\|f\|_{\mathcal{L}_{p}^{\varphi}(\mathcal{B})} \\
& =\sup _{n \geq 0} \sup _{B \in \mathcal{B}_{n}} \varphi(P(B))\left(\frac{1}{P(B)} \int_{B}\left|f(\omega)-E_{n} f(\omega)\right|^{p} d P(\omega)\right)^{1 / p}, \\
\|f\|_{\left(\mathcal{L}_{p}^{\varphi}\right)^{-}} & =\|f\|_{\left(\mathcal{L}_{p}^{\varphi}\right)^{-(\mathcal{B})}} \\
& =\sup _{n \geq 0} \sup _{B \in \mathcal{B}_{n}} \varphi(P(B))\left(\frac{1}{P(B)} \int_{B}\left|f(\omega)-E_{n-1} f(\omega)\right|^{p} d P(\omega)\right)^{1 / p},
\end{aligned}
$$

and define

$$
\begin{gathered}
L_{p}^{\varphi}=L_{p}^{\varphi}(\mathcal{B})=\left\{f \in L_{p}^{0}:\|f\|_{L_{p}^{\varphi}}<\infty\right\}, \\
\mathcal{L}_{p}^{\varphi}=\mathcal{L}_{p}^{\varphi}(\mathcal{B})=\left\{f \in L_{p}^{0}:\|f\|_{\mathcal{L}_{p}^{\varphi}}<\infty\right\}, \\
\left(\mathcal{L}_{p}^{\varphi}\right)^{-}=\left(\mathcal{L}_{p}^{\varphi}\right)^{-}(\mathcal{B})=\left\{f \in L_{p}^{0}:\|f\|_{\left(\mathcal{L}_{p}^{\varphi}\right)^{-}}<\infty\right\} .
\end{gathered}
$$

5.6. Replacing sup in the Morrey norm by other norms. Instead of taking the supremum, Fueto considered to take the $L^{p}$-norm in [21]. Although Feuto worked in the weighted setting, we describe it in the unweighted setting.

Definition 5.10. Let $0<q \leq p<\infty$ and $0<r \leq \infty$. One defines $\mathcal{M}_{q, r}^{p}\left(\mathbb{R}^{n}\right)$ to be the set of all $f \in L_{\mathrm{loc}}^{q}\left(\mathbb{R}^{n}\right)$ for which

$$
\|f\|_{\mathcal{M}_{q, r}^{p}}=\left\|\left\{\left|Q_{\nu m}\right|^{\frac{1}{p}-\frac{1}{q}}\left(\int_{Q_{\nu m}}|f(y)|^{q} d y\right)^{\frac{1}{q}}\right\}_{\nu \in \mathbb{Z}, m \in \mathbb{Z}^{n}}\right\|_{\ell^{r}}
$$

is finite.

See $[6,67]$ for applications to partial differential equations.

5.7. Grandification of the parameter $q$. In addition to generalization of the parameter $p$, we can also grandify the parameter $q$; for $f \in L^{0}\left(\mathbb{R}^{n}\right)$, we define

$$
\|f\|_{\mathcal{M}_{q), \theta}^{p}} \equiv \sup _{x \in \mathbb{R}^{n}, r>0} \sup _{\varepsilon \in(0, q-1)} \varepsilon^{\theta}|Q(x, r)|^{\frac{1}{p}-\frac{1}{q-\varepsilon}}\left(\int_{Q(x, r)}|f(y)|^{q-\varepsilon} d y\right)^{\frac{1}{q-\varepsilon}} .
$$

The space $\mathcal{M}_{q), \theta}^{p}\left(\mathbb{R}^{n}\right)$ collects all $f \in L^{0}\left(\mathbb{R}^{n}\right)$ for which $\|f\|_{\mathcal{M}_{q), \theta}^{p}}$ is finite. See $[58,68,71]$ for more details and variants.

5.8. The case of the variable expoenent. By a variable exponent we mean any measurable function from $\mathbb{R}^{n}$ to a subset of $(-\infty, \infty]$. We define $\|\cdot\|_{L^{p(\cdot)}}$ which is called the variable Lebesgue norm or the Nakano-Luxenburg norm. 
Definition 5.11 (Variable Lebesgue spaces, Variable exponent Lebesgue space). Let

$$
p(\cdot): \mathbb{R}^{n} \rightarrow[1, \infty]
$$

be a measurable function. Then define the variable exponent Lebesgue space $L^{p(\cdot)}\left(\mathbb{R}^{n}\right)$ with variable exponents by

$$
L^{p(\cdot)}\left(\mathbb{R}^{n}\right) \equiv \bigcup_{\lambda>0}\left\{f \in L^{0}\left(\mathbb{R}^{n}\right): \rho_{p}\left(\lambda^{-1} f\right)<\infty\right\},
$$

where

$$
\rho_{p}(f) \equiv\left\|\chi_{p^{-1}(0, \infty)}|f|^{p(\cdot)}\right\|_{1}+\|f\|_{L^{\infty}\left(p^{-1}(\infty)\right)} .
$$

Moreover, for $f \in L^{p(\cdot)}\left(\mathbb{R}^{n}\right)$ one defines the variable Lebesgue norm by

$$
\|f\|_{L^{p(\cdot)}\left(\mathbb{R}^{n}\right)} \equiv \inf \left(\left\{\lambda \in(0, \infty): \rho_{p}\left(\lambda^{-1} f\right) \leq 1\right\} \cup\{\infty\}\right) .
$$

Using this techinique, we can generalize the exponent $q$. See $[37,65,66]$ for example.

\section{ACKNOWLEDGEMENT}

The author is thankful to Professors Eiichi Nakai and Vagif Guliyev for their careful reading.

\section{REFERENCES}

[1] R.A.. Adams and J.J.F. Fournier, Sobolev spaces. Volume 140 of Pure and Applied Mathematics,V Elsevier, Academic Press, New York, 2nd edition, 2003.

[2] A. Akbulut, V. Guliyev and R.Ch. Mustafayev, On the boundedness of the maximal operator and singular integral operators in generalized Morrey spaces, Math. Bohem. 137 (2012), no. $1,27-43$.

[3] A. Akbulut and O. Kuzu, Marcinkiewicz integrals with rough kernel associated with Schrodinger operator on vanishing generalized Morrey spaces, Azerb. J. Math. 4 (2014), no. $1,40-54$.

[4] R. Arai and E. Nakai, Commutators of Calderón-Zygmund and generalized fractional integral operators on generalized Morrey spaces. Rev. Mat. Complut. 31 (2018), no. 2, 287-331.

[5] A.S. Balakishiyev, V.S. Guliyev, F. Gurbuz and A. Serbetci, Sublinear operators with rough kernel generated by Calderón-Zygmund operators and their commutators on generalized local Morrey spaces, J. Inequal. Appl. 2015, 2015:61, 18 pp.

[6] J. Bourgain, On the restriction and multiplier problems in $\mathbb{R}^{3}$, Geometric aspects of functional analysis (1989-90), Lecture Notes in Math., vol. 1469, Springer, Berlin, 1991, 179-191.

[7] W.S. Budhi and J. Lindiarti, Boundedness of multilinear generalized fractional integral operators in generalized Morrey space, Far East J. Math. Sci., 57 (2011), no. 1, 91-104.

[8] Y.P. Chen and Y. Ding, Commutators of parabolic singular integrals on the generalized Morrey spaces, Acta Math. Appl. Sin. Engl. Ser. 30 (2014), no. 2, 367-378.

[9] Y.P. Chen, Y. Ding and X.X. Wang, Commutators of Littlewood-Paley operators on the generalized Morrey space, J. Inequal. Appl. 2010, Art. ID 961502, 20 pp.

[10] F. Chiarenza and M. Frasca, Morrey spaces and Hardy-Littlewood maximal function, Rend. Mat., 7 (1987), 273-279.

[11] F. Deringoz, V.S. Guliyev and S. Samko, Boundedness of the maximal and singular operators on generalized Orlicz-Morrey spaces, Operator theory, operator algebras and applications, 139-158, Oper. Theory Adv. Appl., 242, Birkhäuser/Springer, Basel, 2014. 
[12] F. Deringoz, V. S. Guliyev and S. Samko, Boundedness of the maximal operator and its commutators on vanishing generalized Orlicz-Morrey spaces, Ann. Acad. Sci. Fenn. Math. 40 (2015), no. 2, 535-549.

[13] M.S. Dzhabrailov and S.Z. Khaligova, Anisotropic fractional maximal operator in anisotropic generalized Morrey spaces, J. Math. Res. 4 (2012), no. 6, 109-120.

[14] Eridani and Y. Sawano, Fractional Integral Operators in Generalized Morrey Spaces Defined on Metric Measure Spaces, Proc. A. Razmadze Math. Inst. 158 (2012), 13-24.

[15] A. Eroglu, Boundedness of fractional oscillatory integral operators and their commutators on generalized Morrey spaces, Bound. Value Probl. 2013, 2013:70, 12 pp.

[16] A. Eroglu, V.S. Guliyev, Dzh.V. Azizov, Characterizations of fractional integral operators in generalized Morrey spaces on Carnot groups. (Russian) Mat. Zametki 102 (2017), no. 5, 789-804; translation in Math. Notes 102 (2017), no. 5-6, 722734

[17] Eridani, H. Gunawan and E. Nakai, On generalized fractional integral operators, Sci. Math. Jpn. 60 (2004), 539-550.

[18] Eridani, H. Gunawan, E. Nakai and Y. Sawano, Characterizations for the generalized fractional integral operators on Morrey spaces, Math. Ineq. Appl., 17 (2014), no. 2, 761-777.

[19] Eridani, M. I. Utoyo and H. Gunawan, A characterization for fractional integral operators on generalized Morrey spaces, Anal. Theory Appl. 28 (2012), No. 3, 263-267.

[20] A. Eroglu, T. Gadjiev and F. Namazov, Fractional integral associated to Schrödinger operator on the Heisenberg groups in central generalized Morrey spaces, J. Nonlinear Sci. Appl., 11 (2018), 984-993.

[21] J. Fueto, Norm inequalities in generalized Morrey spaces, J. Fourier Anal. Appl. 20 (2014), no. 4, 896-909.

[22] S. Gala, Y. Sawano and H. Tanaka, A remark on two generalized Orlicz-Morrey spaces, J. Approx. Theory 198 (2015), 1-9.

[23] G.L. Gao and X.M. Wu, Estimates of intrinsic square functions on generalized weighted Morrey spaces, J. Funct. Spaces 2014, Art. ID 168381, 8 pp.

[24] L. Grafakos, Classical Fourier Analysis. Graduate texts in mathematics; 249, New York, Springer, 2008.

[25] L. Grafakos, Modern Fourier Analysis. Graduate texts in mathematics; 250, New York, Springer, 2009.

[26] A. Gogatishvili and R.Ch. Mustafayev, On a theorem of Muchenhoupt-Wheeden in generalized Morrey spaces, Eurasian Math. J. 2 (2011), no. 2, 134-138.

[27] A. Gogatishvili and R.Ch. Mustafayev, Equivalence of norms of Riesz potential and fractional maximal function in generalized Morrey spaces, Collect. Math. 63 (2012), no. 1, $11-28$.

[28] V.S., Guliyev, Integral operators on function spaces on the homogeneous groups and on domains in $\mathbb{R}^{n}$ (Russian). Doctor degree dissertation, Mat. Inst. Steklov, Moscow (1994)

[29] V. S. Guliyev, Boundedness of the maximal, potential and singular operators in the generalized Morrey spaces, J. Inequal. Appl. 2009, Art. ID 503948, 20 pp.

[30] V.S. Guliyev, Local generalized Morrey spaces and singular integrals with rough kernel, Azerb. J. Math. 3 (2013), no. 2, 79-94.

[31] V.S. Guliyev, S.S. Aliyev and T. Karaman, Boundedness of sublinear operators and commutators on generalized Morrey spaces, Abstr. Appl. Anal. vol. 2011, Art. ID 356041, 18 pp. doi:10.1155/2011/356041

[32] V.S. Guliyev, A. Akbulut and Y. Mammadov, Boundedness of fractional maximal operator and their higher order commutators in generalized Morrey spaces on Carnot groups, Acta Math. Sci. Ser. B Engl. Ed. 33 (2013), no. 5, 1329-1346.

[33] V.S. Guliyev, S.S. Aliyev, T. Karaman and P. Shukurov, Boundedness of sublinear operators and commutators on generalized Morrey spaces, Integral Equations and Operator Theory, 71 (3) 2011, 1-29. 
[34] V.S. Guliyev, A. Eroglu and Y.Y. Mammadov, Riesz potential in generalized Morrey spaces on the Heisenberg group. Problems in mathematical analysis, No. 68. J. Math. Sci. (N. Y.) 189 (2013), no. 3, 365-382.

[35] V.S. Guliyev, T.S. Gadjiev and S.S. Aliyev, Interior estimates in generalized Morrey spaces of solutions to nondivergence elliptic equations with VMO coefficients, Dokl. Nats. Akad. Nauk Azerb. 68 (2012), no. 6, [On title page: Vol. 67], 3-10.

[36] V. S. Guliyev, S. G. Hasanov, Y. Sawano and T. Noi, Non-smooth atomic decompositions for generalized Orlicz-Morrey spaces of the third kind, Acta Appl. Math. 145 (2016), 133-174.

[37] V.S. Guliyev, J.J. Hasanov and S. Samko, Boundedness of the maximal, potential and singular operators in the generalized variable exponent Morrey spaces, Math. Scand. 197 (2) (2010) 285-304.

[38] V.S. Guliyev, T. Karaman, A.S. Seymur and P. Shukurov, Boundedness of sublinear operators and commutators on generalized Morrey spaces, Integral Equations and Operator Theory, 71, Issue 3 (2011), 327-355.

[39] V.S. Guliyev and Y.Y. Mammadov, Boundedness of the fractional maximal operator in generalized Morrey space on the Heisenberg group, Indian J. Pure Appl. Math. 44 (2013), no. 2, 185-202.

[40] V.S. Guliyev and R.Ch. Mustafayev, Boundedness of the anisotropic maximal and anisotropic singular integral operators in generalized Morrey spaces, Acta Math. Sin. 27 (2011), no. 12, 2361-2370.

[41] G.S. Guliyev and M.N. Omarova, Higher order commutators of vector-valued intrinsic square functions on vector-valued generalized weighted Morrey spaces, Azerb. J. Math. 4 (2014), no. 2, 64-85.

[42] V. S. Guliyev and M. N. Omarova, Parabolic oblique derivative problem with discontinuous coefficients in generalized weighted Morrey spaces, Open Math. 14 (2016), 49-61.

[43] V.S. Guliyev, M.N. Omarova, M.A. Ragusa and A. Scapellato, Commutators and generalized local Morrey spaces, J. Math. Anal. Appl., 457 (2) (2018), 1388-1402.

[44] G.S. Guliyev, M.N. Omarova and Y. Sawano, Boundedness of intrinsic square functions and their commutators on generalized weighted Orlicz-Morrey spaces, Banach J. Math. Anal. 9 (2015), no. 2, 44-62.

[45] V.S. Guliyev and Y. Sawano, Linear operators on generalized Morrey spaces with nondoubling measures, Publ. Math. Debrecen. 83 (2013), no. 3, 303-327.

[46] V.S. Guliyev and P. Shukurov, Adams type result for sublinear operators generated by Riesz potentials on generalized Morrey spaces, Trans. Natl. Acad. Sci. Azerb. Ser. Phys.-Tech. Math. Sci. 32 (2012), no. 1, Mathematics, 61-70.

[47] V.S. Guliyev and P.S. Shukurov, On the boundedness of the fractional maximal operator, Riesz potential and their commutators in generalized Morrey spaces, Advances in harmonic analysis and operator theory, 175-199, Oper. Theory Adv. Appl., 229, Birkhauser/Springer Basel AG, Basel, 2013.

[48] V.S. Guliyev and K.R. Rahimova, Parabolic fractional maximal operator in parabolic generalized Morrey spaces, Proc. Inst. Math. Mech. Natl. Acad. Sci. Azerb. 37 (2012), 51-66.

[49] H. Gunawan, A note on the generalized fractional integral operators, J. Indones. Math. Soc., 9 (2003), 39-43.

[50] H. Gunawan and Eridani, Fractional integrals and generalized Olsen inequalities, Kyungpook Math. J., 49 (2009), 31-39.

[51] D. I. Hakim, E. Nakai, and Y. Sawano, Generalized Fractional Maximal Operators and Vector-valued Inequalities on Generalized Orlicz-Morrey Spaces, Rev. Mat. Complut. 29 (2016), no. 1, 59-90.

[52] D. I. Hakim and Y. Sawano, Interpolation of generalized Morrey spaces, Rev. Mat. Complut. 29 (2016), no. 2, 295-340.

[53] D.I. Hakim, Y. Sawano and T. Shimomura, Boundedness of generalized fractional integral operators from the Morrey space $L^{1, \phi}(X, \mu)$ to the Campanato space $\mathcal{L}^{1, \psi}(X, \mu)$ over nondoubling measure spaces, Azerb. J. Math. 6 (2016), no. 2, 117-127. 
[54] G.H. Hardy and J. Littlewood, Some properties of fractional integrals I, Math. Z. 27 (1927), 565-606.

[55] G.H. Hardy and J. Littlewood, Some properties of fractional integrals II, Math. Z. 34 (1932), 403-439.

[56] K.P. Ho, Strong maximal operator and singular integral operators in weighted Morrey spaces on product domains, Math. Nachr. 290 (2017), no. 16, 2629-2640.

[57] T. Karaman, V.S. Guliyev and A. Serbetci, Boundedness of sublinear operators generated by Calderon-Zygmund operators on generalized weighted Morrey spaces, An. -tiin-. Univ. Al. I. Cuza Ia-i. Mat. (N.S.) 60 (2014), no. 1, 227-244.

[58] V. Kokilashvili, A. Meskhi and H. Rafeiro, Riesz type potential operators in generalized grand Morrey spaces, Georgian Math. J. 20 (2013), no. 1, 43-64.

[59] V. Kokilashvili, A. Meskhi and H. Rafeiro, Estimates for nondivergence elliptic equations with VMO coefficients in generalized grand Morrey spaces, Complex Var. Elliptic Equ. 59 (2014), no. 8, 1169-1184.

[60] Y. Komori and T. Mizuhara, Factorization of functions in $H^{1}\left(\mathbb{R}^{n}\right)$ and generalized Morrey spaces, Math. Nachr. 279 (2006), no. 5-6, 619-624.

[61] K. Kurata, S. Nishigaki and S. Sugano, Boundedness of integral operators on generalized Morrey spaces and its application to Schrodinger operators, Proc. Amer. Math. Soc. 128 (2000) no. 4, 1125-1134.

[62] K. Kurata and S. Sugano, A remark on estimates for uniformly elliptic operators on weighted $L^{p}$ spaces and Morrey spaces, Math. Nachr., 209 (2000), 137-150.

[63] G.H. Liu and L.S. Shu, Boundedness for the commutator of fractional integral on generalized Morrey space in nonhomogeneous space, Anal. Theory Appl. 27 (2011), no. 1, 51-58.

[64] D. Lukkassen, A. Meidell, L.E. Persson and N. Samko, Hardy and singular operators in weighted generalized Morrey spaces with applications to singular integral equations, Math. Methods Appl. Sci. 35 (2012), no. 11, 1300-1311.

[65] D. Lukkassen, L.E. Persson, S. Samko and P. Wall, Weighted Hardy-type inequalities in variable exponent Morrey-type spaces, J. Funct. Spaces Appl. 2013, Art. ID 716029, 11 pp.

[66] P. Long and H. Han, Characterizations of some operators on the vanishing generalized Morrey spaces with variable exponent, J. Math. Anal. Appl. 437 (2016), no. 1, 419-430.

[67] S. Masaki and J. Segata, Existence of a minimal non-scattering solution to the masssubcritical generalized Kortewegde Vries equation, Ann. Inst. H. Poincar Anal. Non Linaire 35 (2018), no. 2, 283-326.

[68] A. Meskhi and Y. Sawano, Density, duality and preduality in grand variable exponent Lebesgue and Morrey spaces, Mediterr. J. Math. 15 (2018), no. 3, Art. 100, 15 pp.

[69] T. Mizuhara, Boundedness of some classical operators on generalized Morrey spaces, Harmonic analysis (Sendai, 1990), 183-189, ICM-90 Satell. Conf. Proc., Springer, Tokyo, 1991.

[70] Y. Mizuta, E. Nakai, T. Ohno and T. Shimomura, Boundedness of fractional integral operators on Morrey spaces and Sobolev embeddings for generalized Riesz potentials, J. Math. Soc. Japan 62 (2010), no. 3, 707-744.

[71] Y. Mizuta and T. Ohno, Trudinger's exponential integrability for Riesz potentials of functions in generalized grand Morrey spaces, J. Math. Anal. Appl. 420 (2014), no. 1, 268-278.

[72] Y. Mizuta, T. Shimomura and T. Sobukawa, Sobolev's inequality for Riesz potentials of functions in non-doubling Morrey spaces, Osaka J. Math. 46 (2009), no. 1, 255-271.

[73] E. Nakai, Hardy-Littlewood maximal operator, singular integral operators and the Riesz potentials on generalized Morrey spaces, Math. Nachr. 166 (1994), 95-103.

[74] E. Nakai, A characterization of pointwise multipliers on the Morrey spaces, Sci. Math. 3 (2000), 445-454.

[75] E. Nakai, Orlicz-Morrey spaces and the Hardy-Littlewood maximal function, Studia Math. 188 (2008), no. 3, 193-221.

[76] E. Nakai, Generalized fractional integrals on generalized Morrey spaces, Math. Nachr. 287 (2014), no. 2-3, 339-351. 
[77] E. Nakai and G. Sadasue, Martingale Morrey-Campanato spaces and fractional integrals, J. Funct. Spaces Appl. 2012, Art. ID 673929, 29 pp

[78] E. Nakai and T. Sobukawa, $B^{u, w}$-function spaces and their interpolation, Tokyo J. Math. 39 (2016), no. 2, 483-516.

[79] S. Nakamura, Generalized weighted Morrey spaces and classical operators, Math. Nachr. 289 (2016), no. 17-18, 2235-2262.

[80] S. Nakamura, T. Noi and Y. Sawano. Generalized Morrey spaces and trace operator, Science China Mathematics, 59 (2016), no. 2, 281-336.

[81] S. Nakamura and Y. Sawano, The singular integral operator and its commutator on weighted Morrey spaces, Collect. Math. 68 (2017), no. 2, 145-174.

[82] J. Neveu, Discrete-parameter martingales, North-Holland, Amsterdam, 1975. ISBN 0720428106

[83] Y. Pan, C. Li and X. Wang, Boundedness of oscillatory integrals with variable CalderonZygmund kernel on weighted Morrey spaces, J. Funct. Spaces Appl. 2013, Art. ID 946435, 5 pp.

[84] J. Peetre, On the theory of $\mathcal{L}_{p, \lambda}$ spaces, J. Funct. Anal. 4 (1969), 71-87.

[85] C. Pérez, Sharp $L^{p}$-weighted Sobolev inequalities, Ann. Inst. Fourier (Grenoble) 45 (1995), 809-824.

[86] L.E. Persson and N. Samko, Weighted Hardy and potential operators in the generalized Morrey spaces, J. Math. Anal. Appl. 377 (2011), no. 2, 792-806.

[87] M. Rosenthal and H.J. Schmeisser, The boundedness of operators in Muckenhoupt weighted Morrey spaces via extrapolation techniques and duality, Rev. Mat. Complut. 29 (2016), no. 3, 623-657.

[88] N. Samko, Maximal, potential and singular operators in vanishing generalized Morrey spaces, J. Global Optim. 57 (2013), no. 4, 1385-1399.

[89] N. Samko, Weighted Hardy operators in the local generalized vanishing Morrey spaces, Positivity 17 (2013), no. 3, 683-706.

[90] Y. Sawano, $l^{q}$-valued extension of the fractional maximal operators for non-doubling measures via potential operators, Int. J. Pure Appl. Math. 26 (2006), no. 4, 505-523.

[91] Y. Sawano, Generalized Morrey Spaces for non-doubling measures, Nonlinear Differ. Equ. Appl., 15 (2008), 413-425.

[92] Y. Sawano, Theory of Besov spaces, Development in Mathematics, Springer, 56.

[93] Y. Sawano and T. Shimomura, Sobolev embeddings for generalized Riesz potentials of functions in Morrey spaces $L^{(1, \phi)}(G)$ over nondoubling measure spaces, J. Funct. Spaces Appl. 2013, Art. ID 984259, 12 pp.

[94] Y. Sawano and T. Shimomura, Sobolev's inequality for Riesz potentials of functions in generalized Morrey spaces with variable exponent attaining the value 1 over non-doubling measure spaces, J. Inequal. Appl. 2013, 2013:12, 19 pp.

[95] Y. Sawano and T. Shimomura, Sobolev embeddings for Riesz potentials of functions in nondoubling Morrey spaces of variable exponents, Collect. Math. 64 (2013), no. 3, 313-350.

[96] Y. Sawano and T. Shimomura, Boundedness of the generalized fractional integral operators on generalized Morrey spaces over metric measure spaces, Z. Anal. Anwend. 36 (2017), no. 2, 159-190.

[97] Y. Sawano and T. Shimomura, Generalized fractional integral operators over non-doubling metric measure spaces, Integral Transforms Spec. Funct. 28 (2017), no. 7, 534-546.

[98] Y. Sawano and T. Shimomura, Generalized fractional integral operators on generalized Orlicz-Morrey spaces of the second kind over non-doubling metric measure spaces, Georgian Math. J. 25 (2018), no. 2, 303-311.

[99] Y. Sawano, T. Shimomura and H. Tanaka, A remark on modified Morrey spaces on metric measure spaces, Hokkaido Math. J. 47 (2018) 1-15.

[100] Y. Sawano, S. Sugano and H. Tanaka, A note on generalized fractional integral operators on generalized Morrey spaces, Bound. Value Probl. 2009, Art. ID 835865, 18 pp. 
[101] Y. Sawano, S. Sugano and H. Tanaka, Generalized fractional integral operators and fractional maximal operators in the framework of Morrey spaces, Trans. Amer. Math. Soc., 363 (2011), no. 12, 6481-6503.

[102] Y. Sawano, S. Sugano and H. Tanaka, Olsen's inequality and its applications to Schrödinger equations, Suurikaiseki Kôkyûroku Bessatsu B26, (2011) 51-80.

[103] Y. Sawano, S. Sugano and H. Tanaka, A note on generalized fractional integral operators on Orlicz-Morrey spaces, Potential Anal. 36 (2012), no. 4, 517-556.

[104] Y. Sawano and H. Tanaka, Morrey spaces for non-doubling measures, Acta Math. Sinica, 21 (2005), no. 6, 1535-1544.

[105] Y. Sawano and H. Wadade, On the Gagliardo-Nirenberg type inequality in the critical Sobolev-Morrey space, J. Fourier Anal. Appl. 19 (2013), no. 1, 20-47.

[106] Y. Sawano and H. Yoshida, A predual of a predual of $B_{\sigma}$ and its applications to commutators, Sci. China Math. 61 (2018), 1437-1472.

[107] Y.L. Shi and X. Tao, Multilinear Riesz potential operators on Herz-type spaces and generalized Morrey spaces, Hokkaido Math. J. 38 (2009), no. 4, 635-662.

[108] Y.L. Shi and X. Tao, Some multi-sublinear operators on generalized Morrey spaces with non-doubling measures, J. Korean Math. Soc. 49 (2012), no. 5, 907-925.

[109] S. Shirai, Notes on commutators of fractional integral operators on generalized Morrey spaces, Sci. Math. Jpn. 63 (2006), no. 2, 241-246.

[110] I. Sihwaningrum, S. Maryani and H. Gunawan, Weak type inequalities for fractional integral operators on generalized non-homogeneous Morrey spaces, Anal. Theory and Appl., 28 (2012) No. 1, 65-72.

[111] S. Sobolev, On a theorem in functional analysis (Russian), Math. Sob. 46 (1938), 471-490.

[112] L.G. Softova, Singular integrals and commutators in generalized Morrey spaces, Acta Math. Sin. 22 (2006), no. 3, 757-766.

[113] L.G. Softova, Parabolic oblique derivative problem with discontinuous coefficients in generalized Morrey spaces, Ric. Mat. 62 (2013), no. 2, 265-278.

[114] L.G. Softova, The Dirichlet problem for elliptic equations with VMO coefficients in generalized Morrey spaces. Advances in harmonic analysis and operator theory, 371-386, Oper. Theory Adv. Appl., 229, Birkhauser/Springer Basel AG, Basel, 2013.

[115] E.M. Stein, Singular integral and differential property of functions. Princeton: Princeton University Press, 1970.

[116] E.M. Stein, Harmonic Analysis: Real-Variable Methods, Orthogonality, and Oscillatory Integrals. Princeton Univ. Press, 1993.

[117] S. Sugano, Some inequalities for generalized fractional integral operators on generalized Morrey spaces, Math. Inequal. Appl., 14 (2011), no.4, 849-865.

[118] S. Sugano and H. Tanaka, Boundedness of fractional integral operators on generalized Morrey spaces, Sci. Math. Jpn., 58 (2003), no. 3, 531-540 (Sci. Math. Jpn. Online 8 (2003), 233-242).

[119] T. Suzuki and H. Wadade, Optimal embeddings on critical Sobolev-Lorentz spaces into generalized Morrey spaces, Adv. Math. Sci. Appl. 22 (2012), no. 1, 225-238.

[120] X.X. Tao and S. He, The boundedness of multilinear operators on generalized Morrey spaces over the quasi-metric space of non-homogeneous type, J. Inequal. Appl. 2013, 2013:330, 15 pp.

[121] S.S. Volosivets, Hausdorff operator of special kind in Morrey and Herz $p$-adic spaces, $p$-Adic Numbers Ultrametric Anal. Appl. 4 (2012), no. 3, 222-230.

[122] H. Wang, Boundedness of intrinsic square functions on generalized Morrey spaces, Georgian Math. J. 21 (2014), no. 3, 351-367.

[123] N. Wei, P.C. Niu, S. Tang and M. Zhu, Estimates in generalized Morrey spaces for nondivergence degenerate elliptic operators with discontinuous coefficients, Rev. R. Acad. Cienc. Exactas Fis. Nat. Ser. A Math. RACSAM 106 (2012), no. 1, 1-33.

[124] X. Wu and T.T. Zheng, Commutators of intrinsic square functions on generalized Morrey spaces, J. Inequal. Appl. 2014, 2014:128, 12 pp 
[125] Da Yang, Do Yang and G. Hu, The Hardy space $H^{1}$ with non-doubling measures and their applications, Lecture Notes in Mathematics, 2084, Springer, Cham, 2013.

[126] W. Yuan, Y. Lu and D. Yang, Fractional Hajłasz-Morrey-Sobolev spaces on quasi-metric measure spaces, Studia Math. 226 (2015), 95-122.

[127] X. Yu and X.X. Tao, Boundedness of multilinear operators on generalized Morrey spaces, Appl. Math. J. Chinese Univ. Ser. B 29 (2014), no. 2, 127-138.

[128] L. Zhang, Y. Jiang, Y. Sheng and J. Zhou, Parabolic equations with VMO coefficients in generalized Morrey spaces, Acta Math. Sin. 26 (2010), no. 1, 117-130. 